\title{
Assembly of a Metalloporphyrin-Polyoxometalate Hybrid Material for Highly Efficient Activation of Molecular Oxygen
}

Shu-Lan Zhu, Xuan Xu, Sha Ou, Min Zhao, Wei-Long He and Chuan-De Wu*

State Key Laboratory of Silicon Materials, Center for Chemistry of High-Performance \& Novel Materials, Department of Chemistry, Zhejiang University, Hangzhou, 310027, P. R. China

Figures:

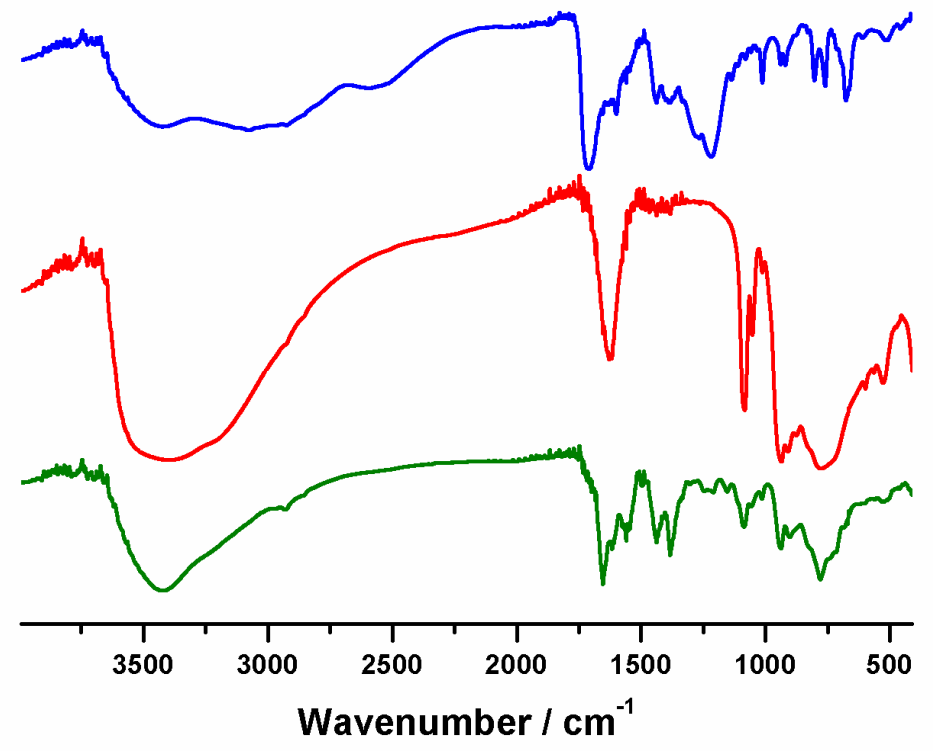

Figure S1. FT-IR spectra of $\mathrm{Mn}-\mathrm{H}_{8} \mathrm{OCPP}$ (blue), $\left\{\mathrm{Gd}_{4} \mathrm{Co}_{6} \mathrm{P}_{4} \mathrm{~W}_{30}\right\}$ (red) and $\mathbf{1}$ (olive).

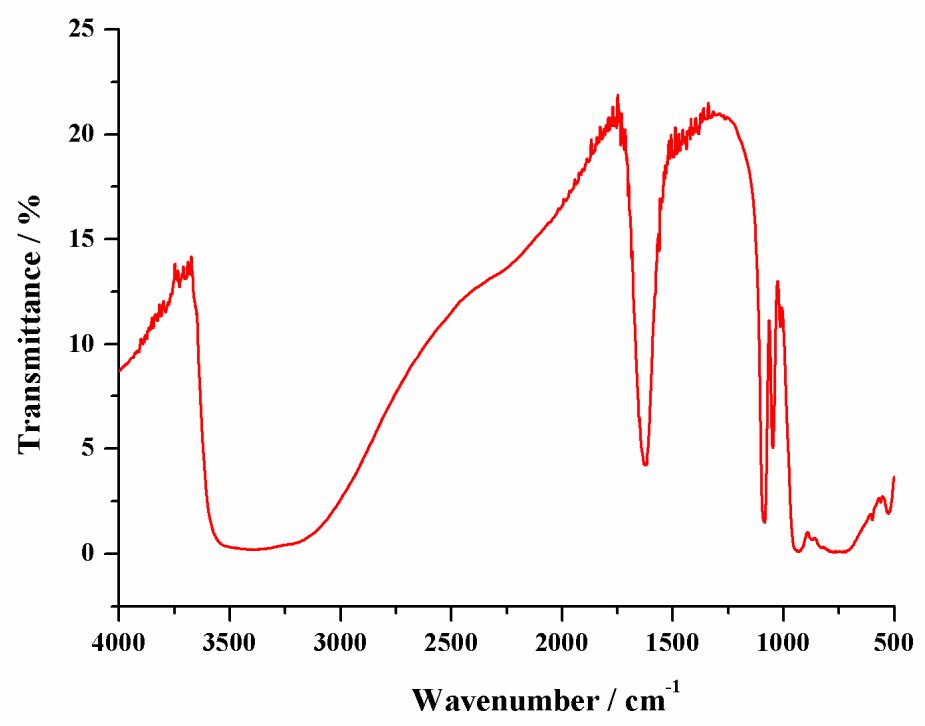

Figure S2. FT-IR spectrum of $\left\{\mathrm{Gd}_{4} \mathrm{Mn}_{6} \mathrm{P}_{4} \mathrm{~W}_{30}\right\}$. 


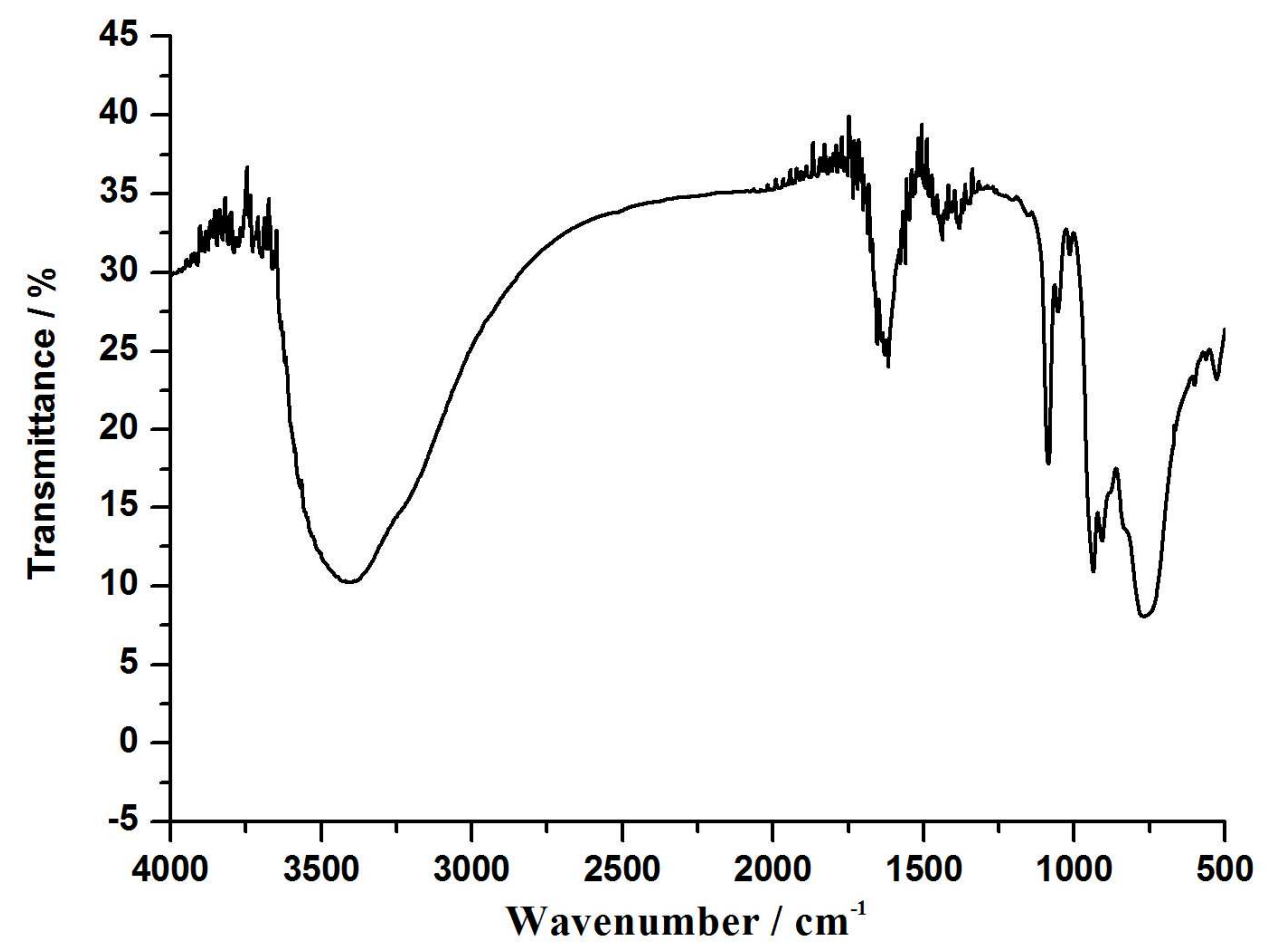

Figure S3. FT-IR spectrum of recovered 1 after catalysis.

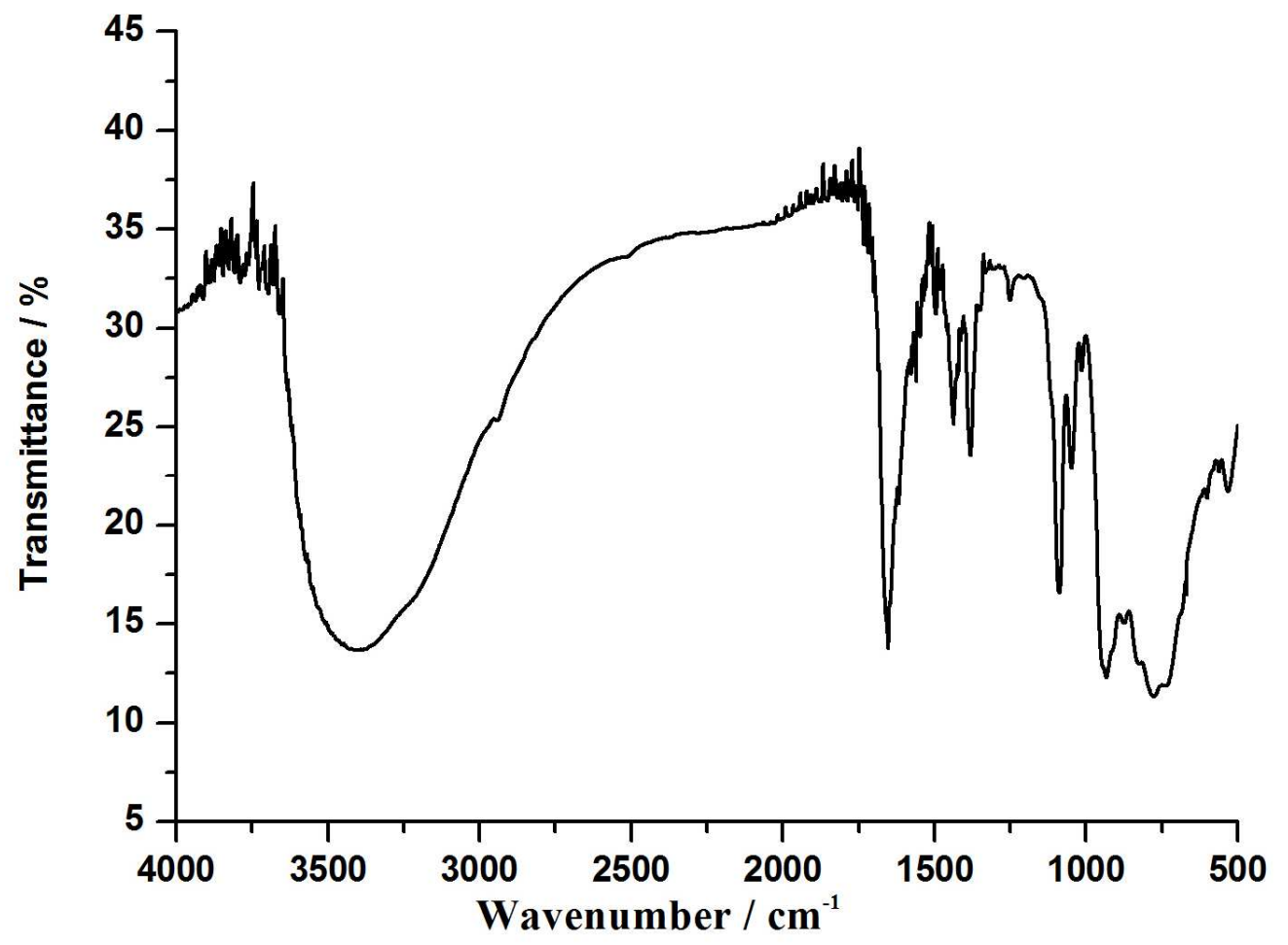

Figure S4. FT-IR spectrum of MnCo-1. 


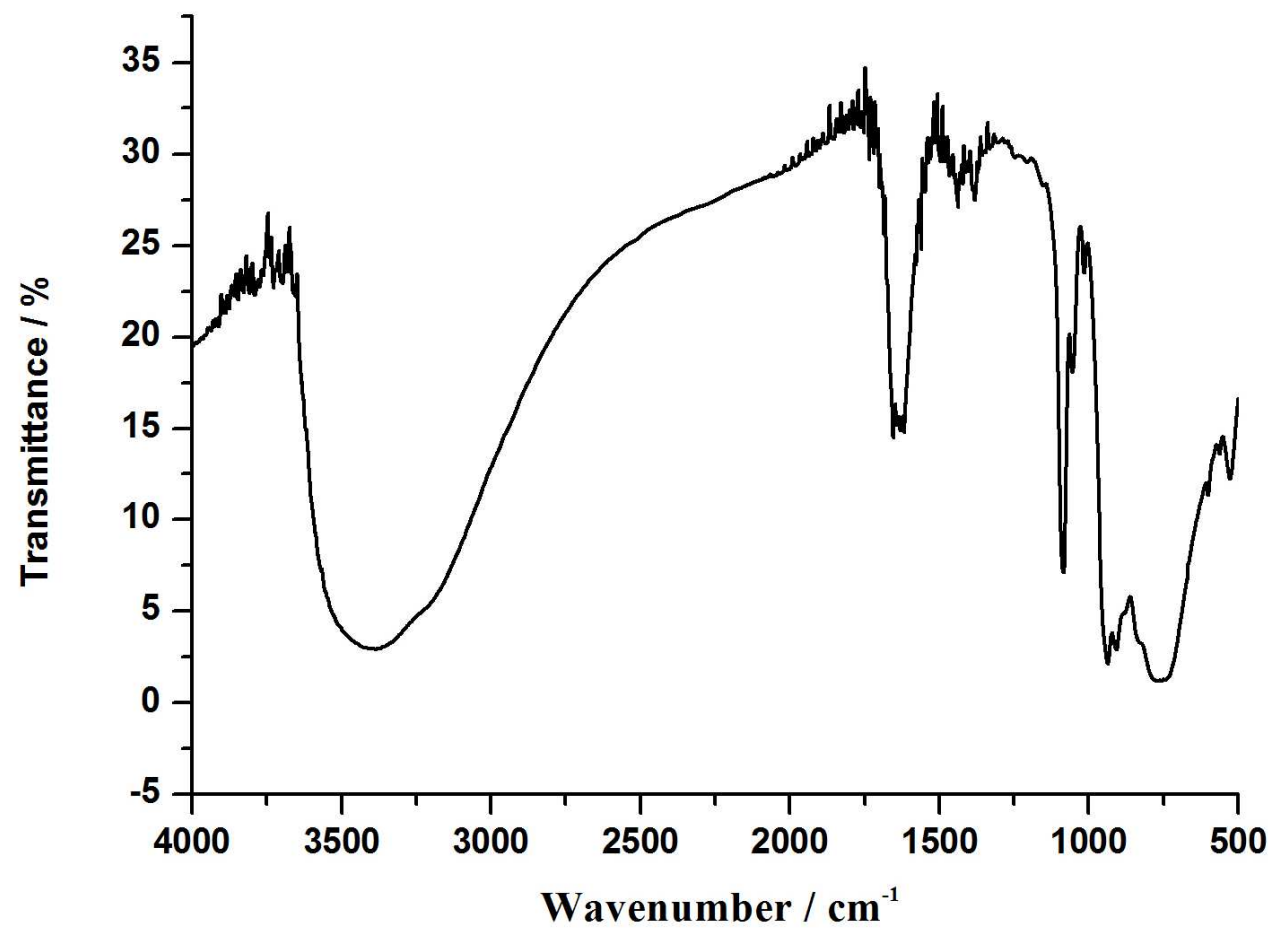

Figure S5. FT-IR spectrum of CoCo-1.

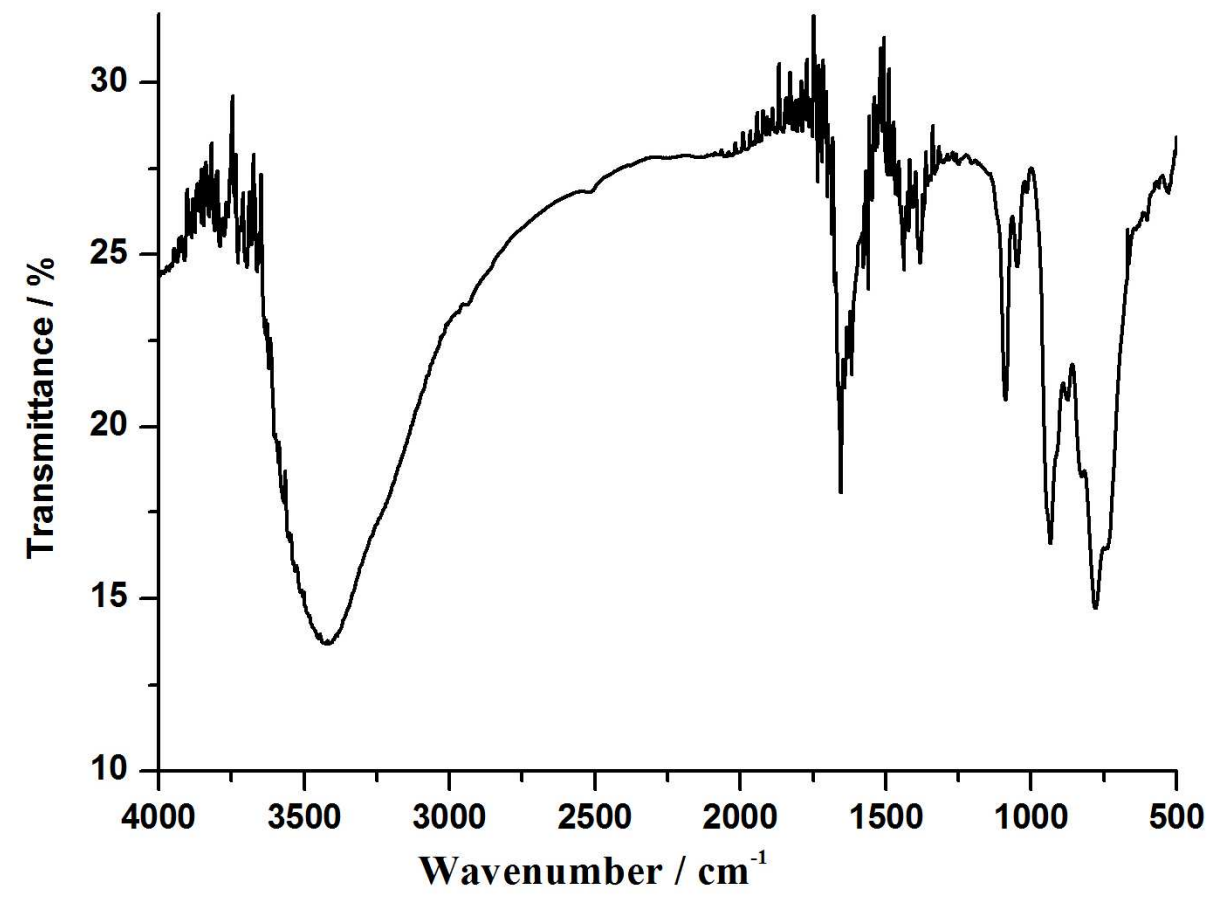

Figure S6. FT-IR spectrum of MnMn-1. 

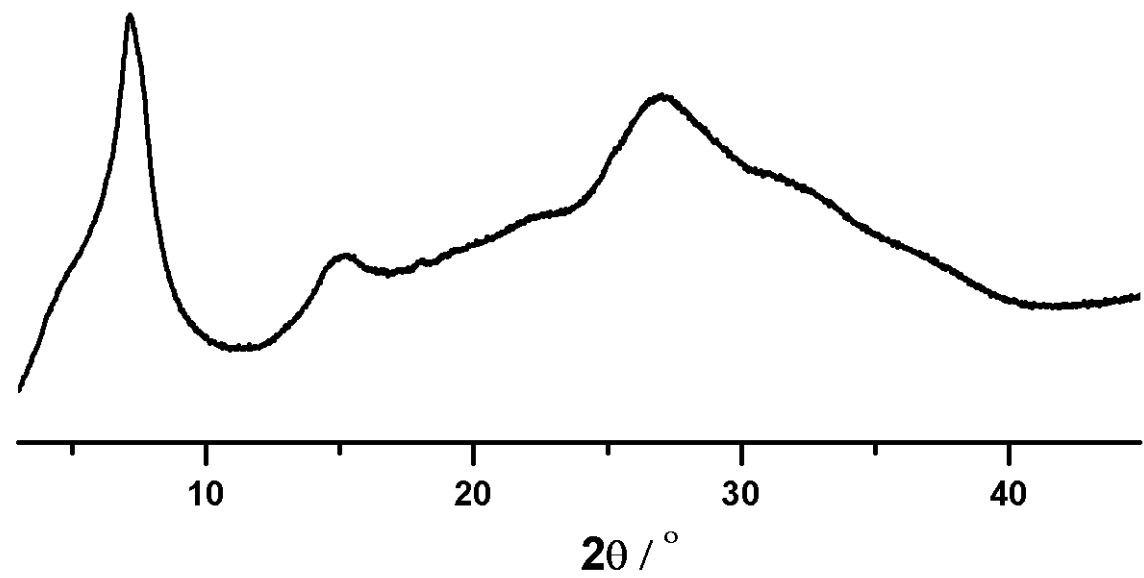

Figure S7. PXRD pattern for 1.
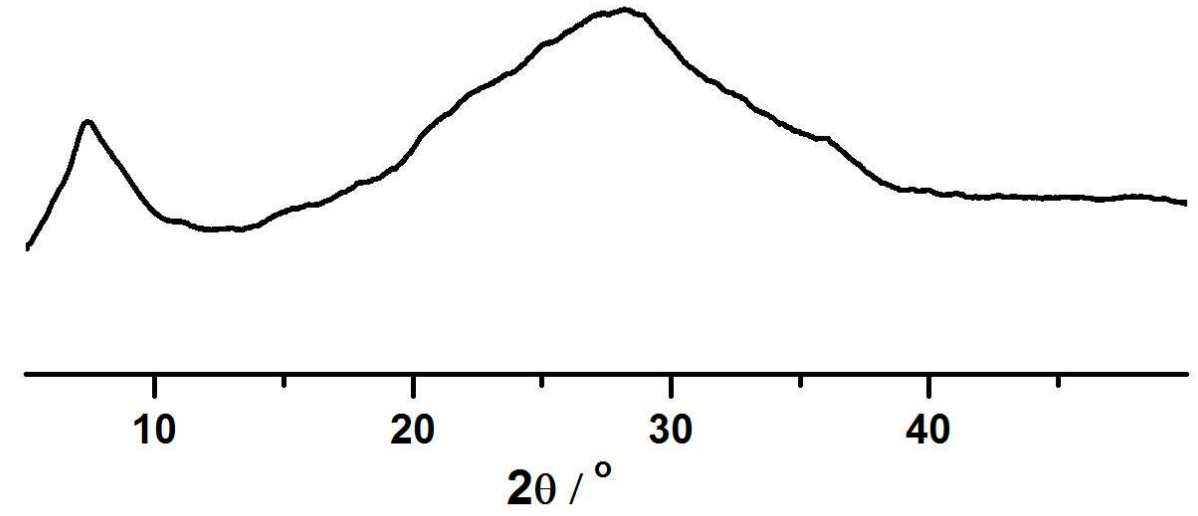

Figure S8. PXRD pattern for recovered 1 after catalysis.

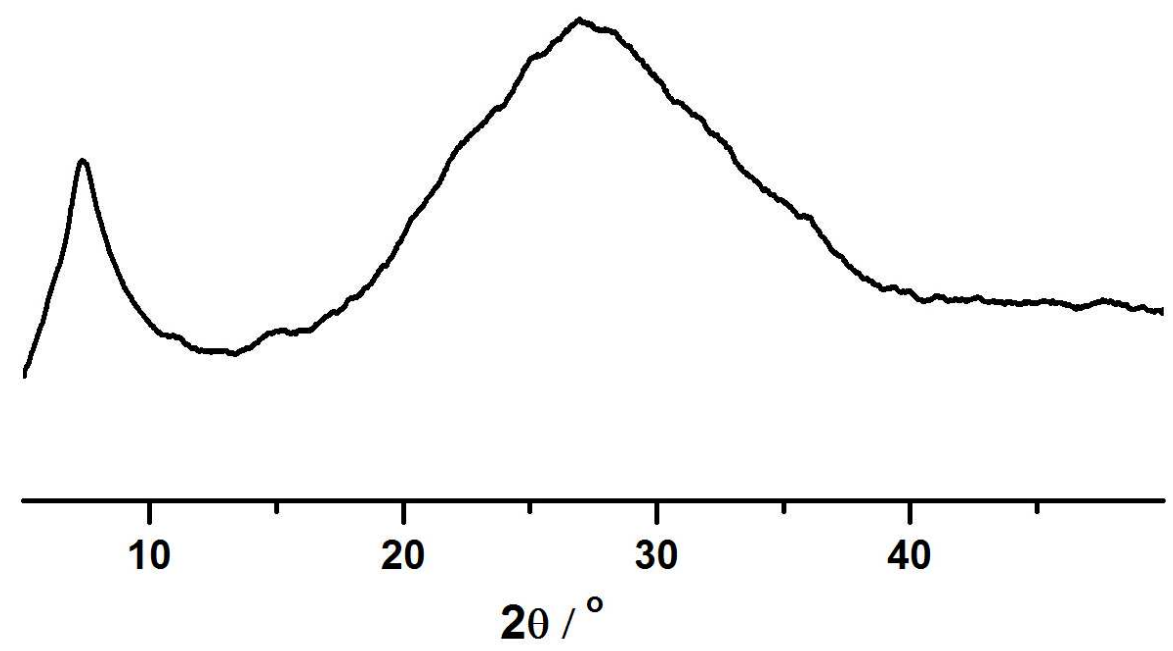

Figure S9. PXRD pattern for MnCo-1. 

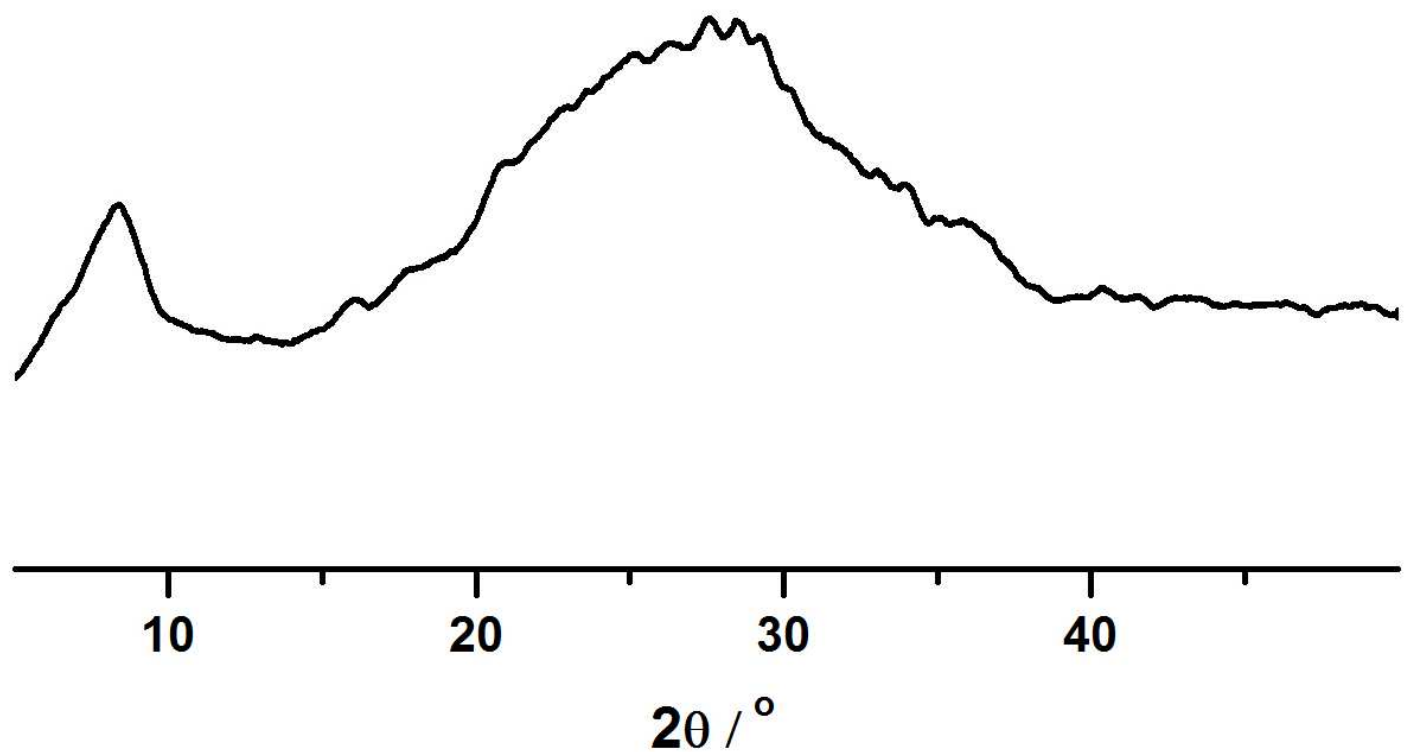

Figure S10. PXRD pattern for CoCo-1.
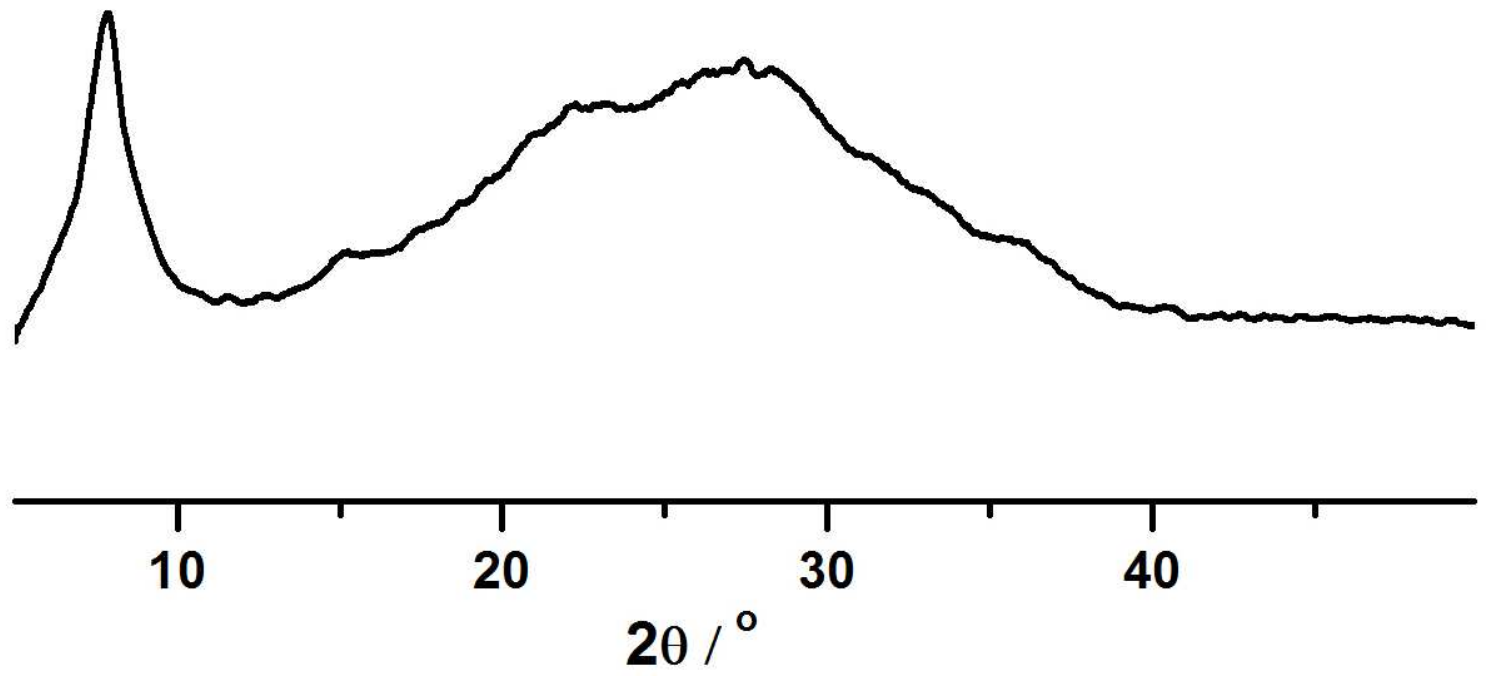

Figure S11. PXRD pattern for MnMn-1. 


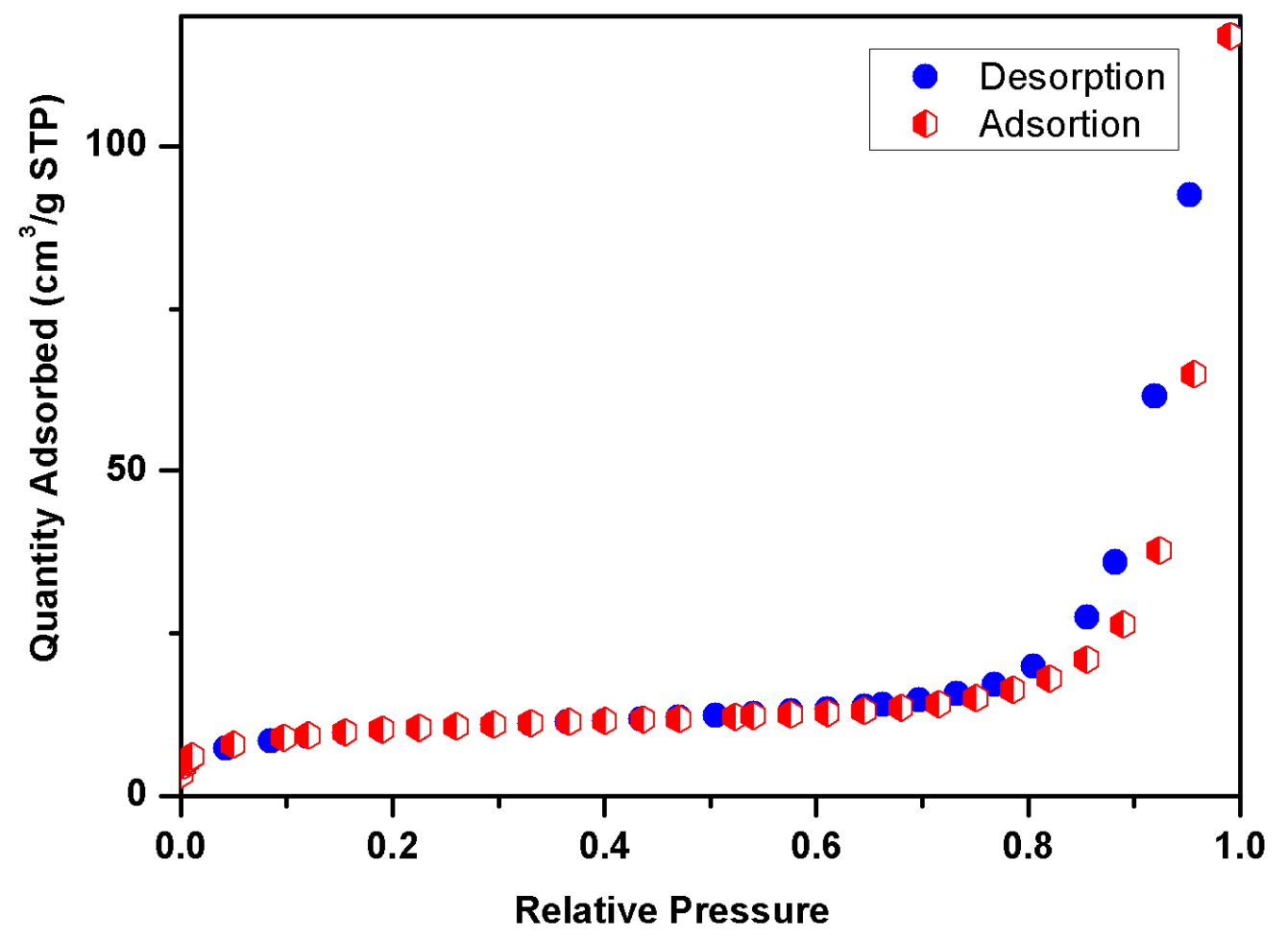

Figure S12. $\mathrm{N}_{2}$ sorption isotherms of 1 at $77 \mathrm{~K}$.

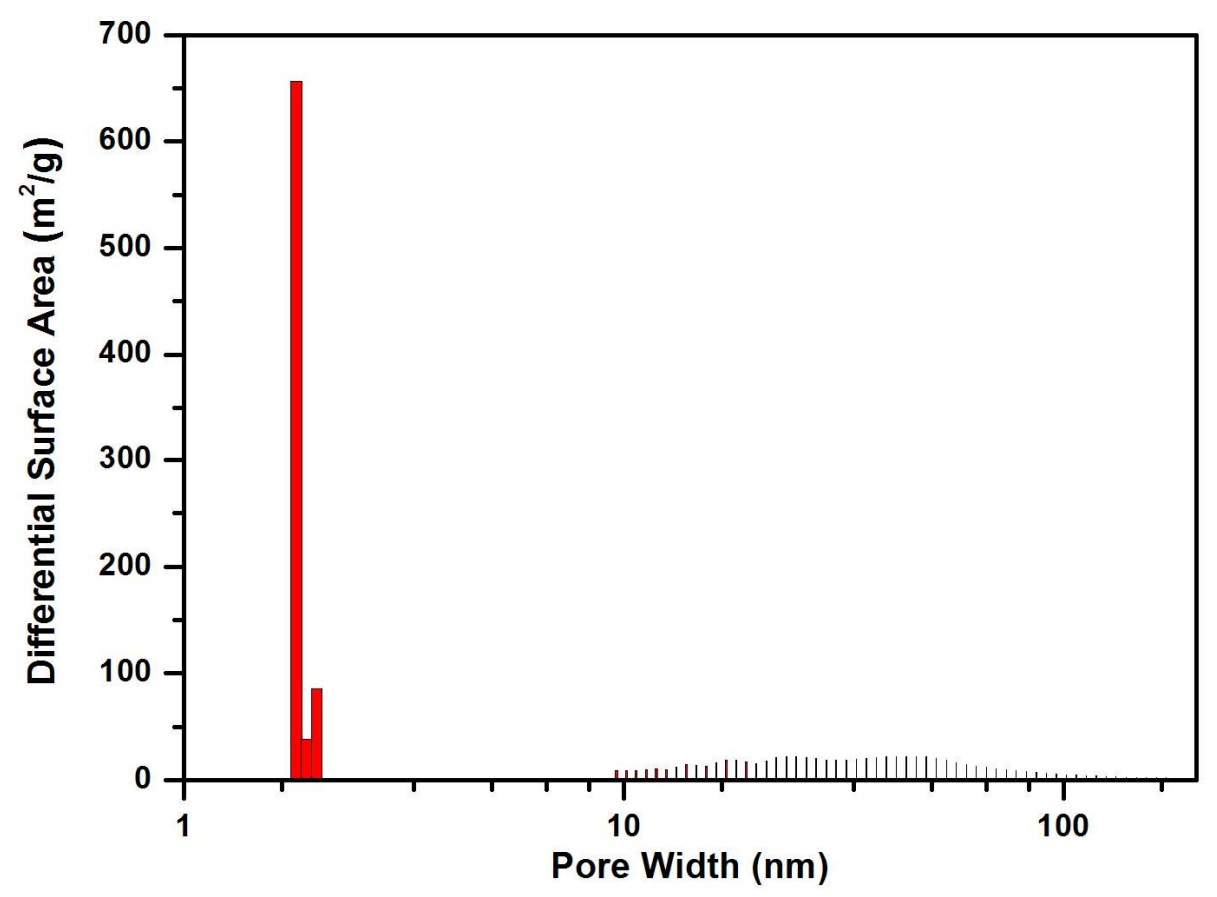

Figure S13. Pore-size distribution of 1, calculated by using DFT method. 


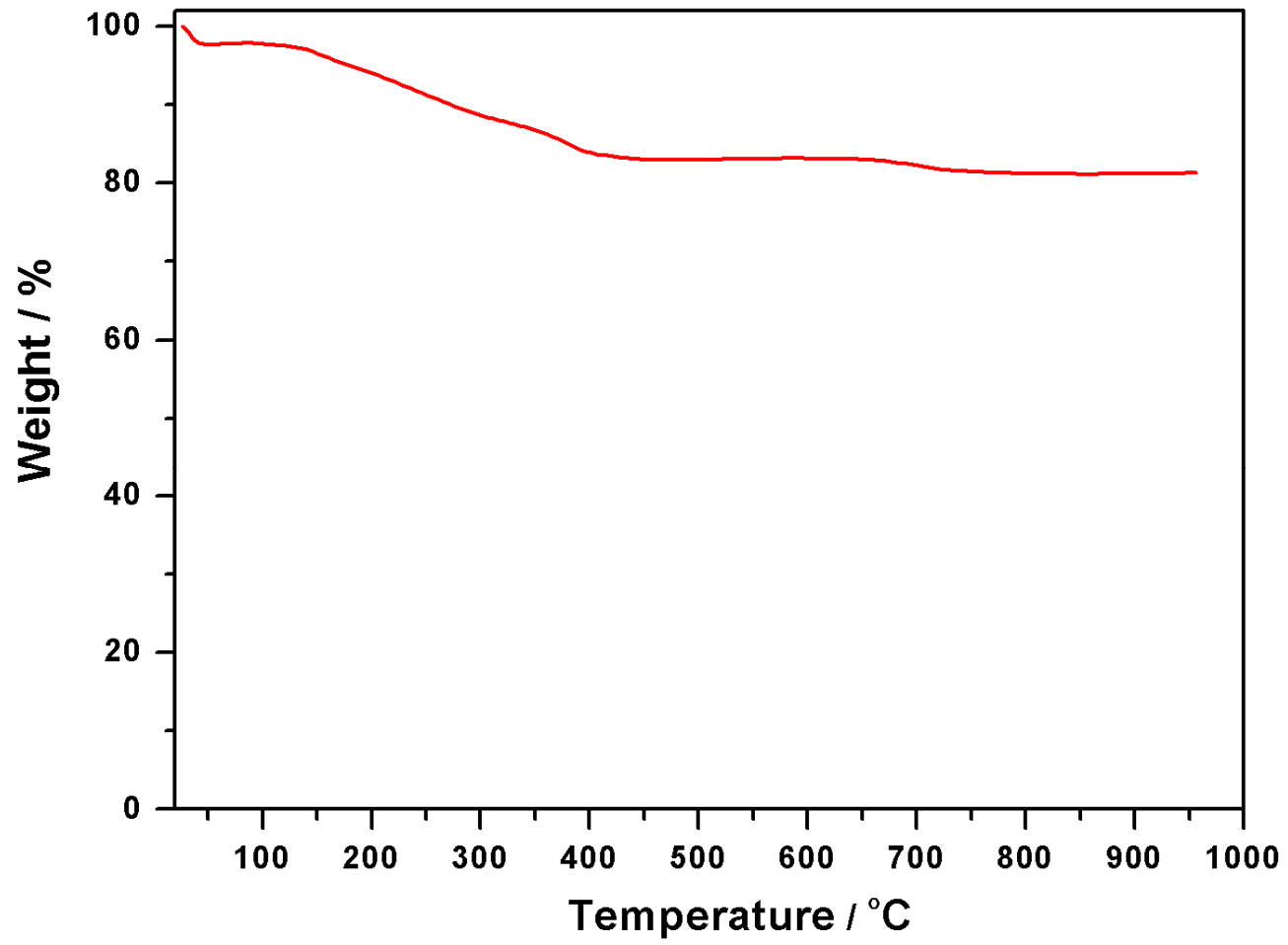

Figure S14. TGA curve for $\left\{\mathrm{Gd}_{4} \mathrm{Co}_{6} \mathrm{P}_{4} \mathrm{~W}_{30}\right\}$.

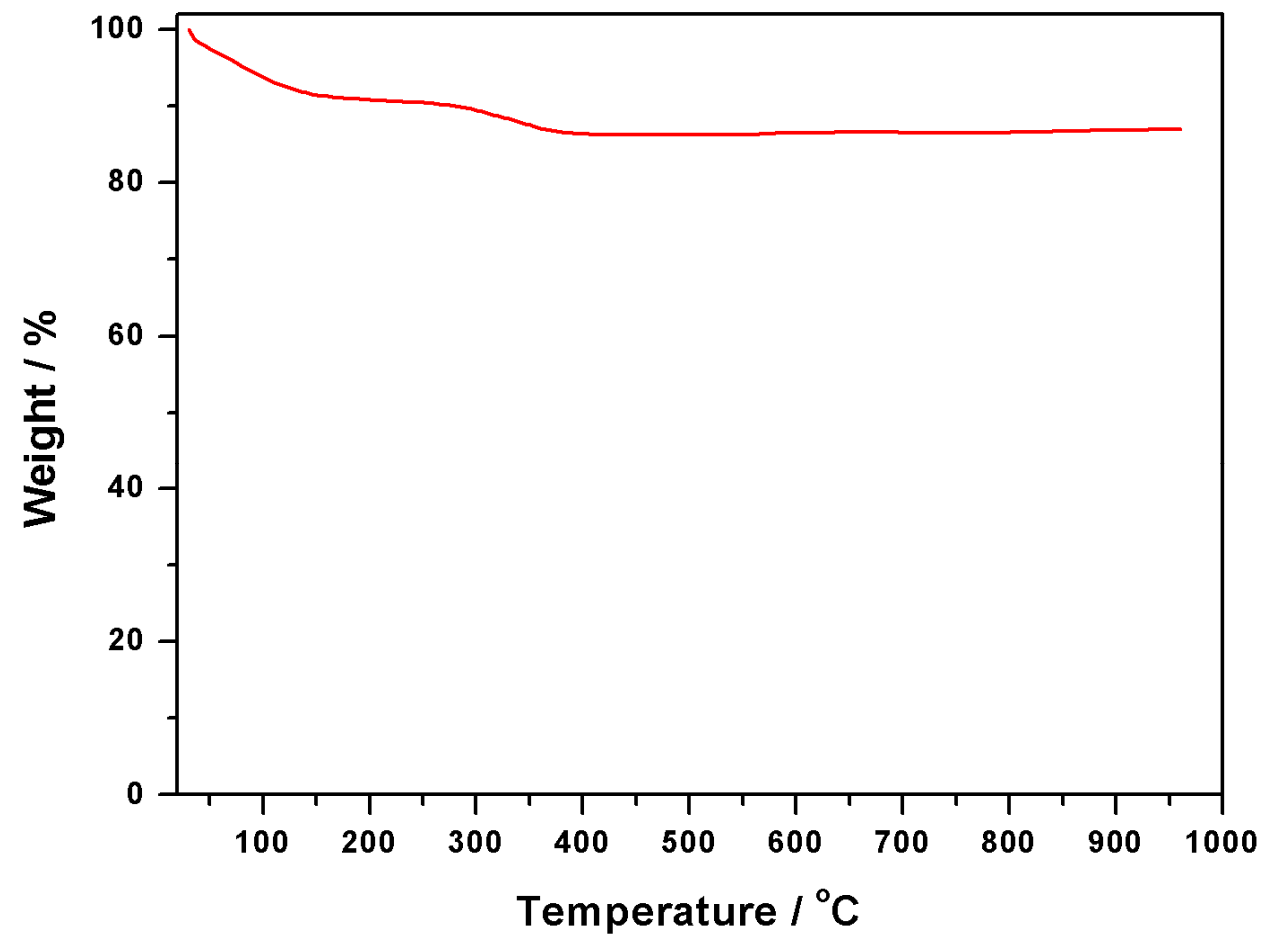

Figure S15. TGA curve for $\left\{\mathrm{Gd}_{4} \mathrm{Mn}_{6} \mathrm{P}_{4} \mathrm{~W}_{30}\right\}$. 


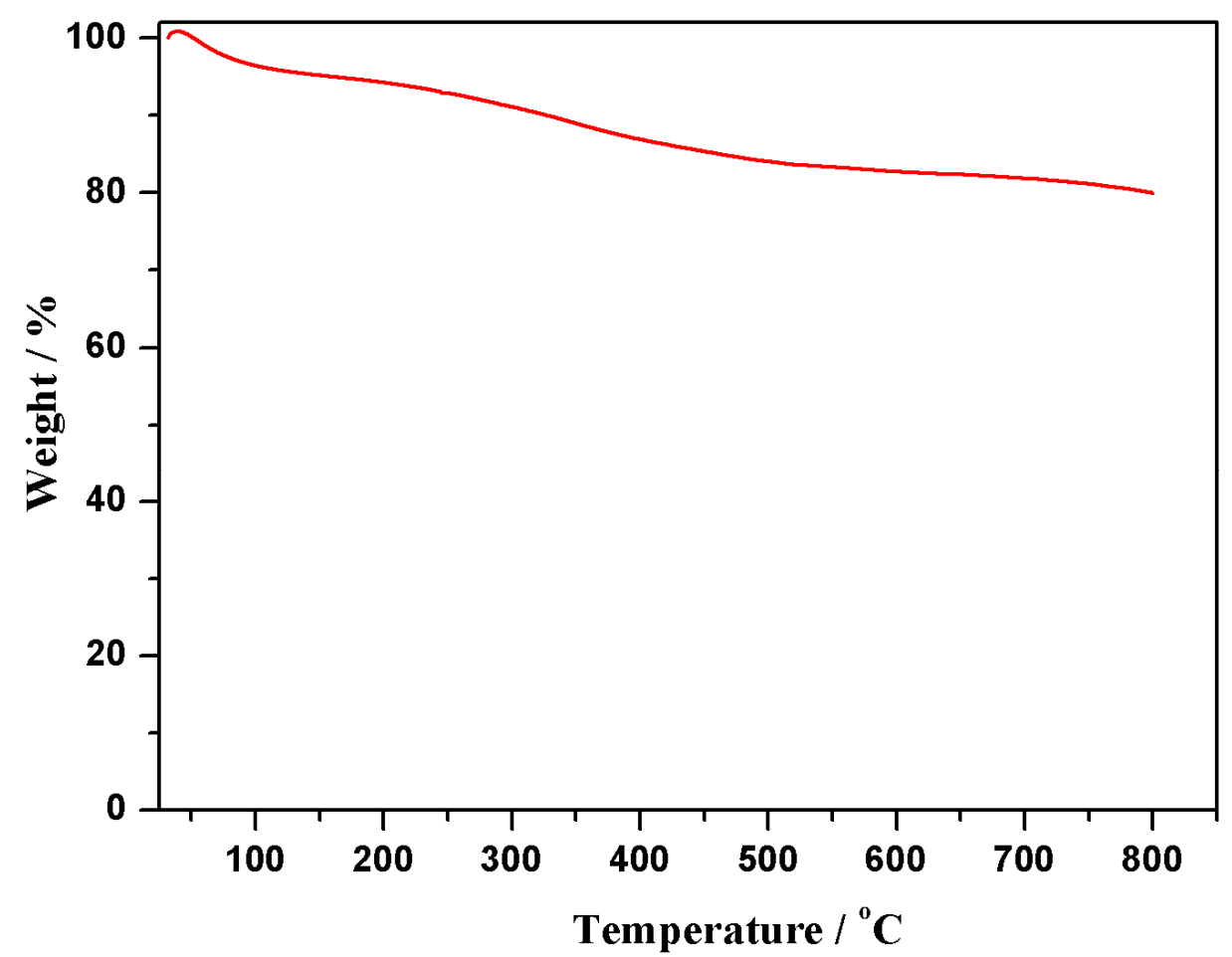

Figure S16. TGA curve for 1.

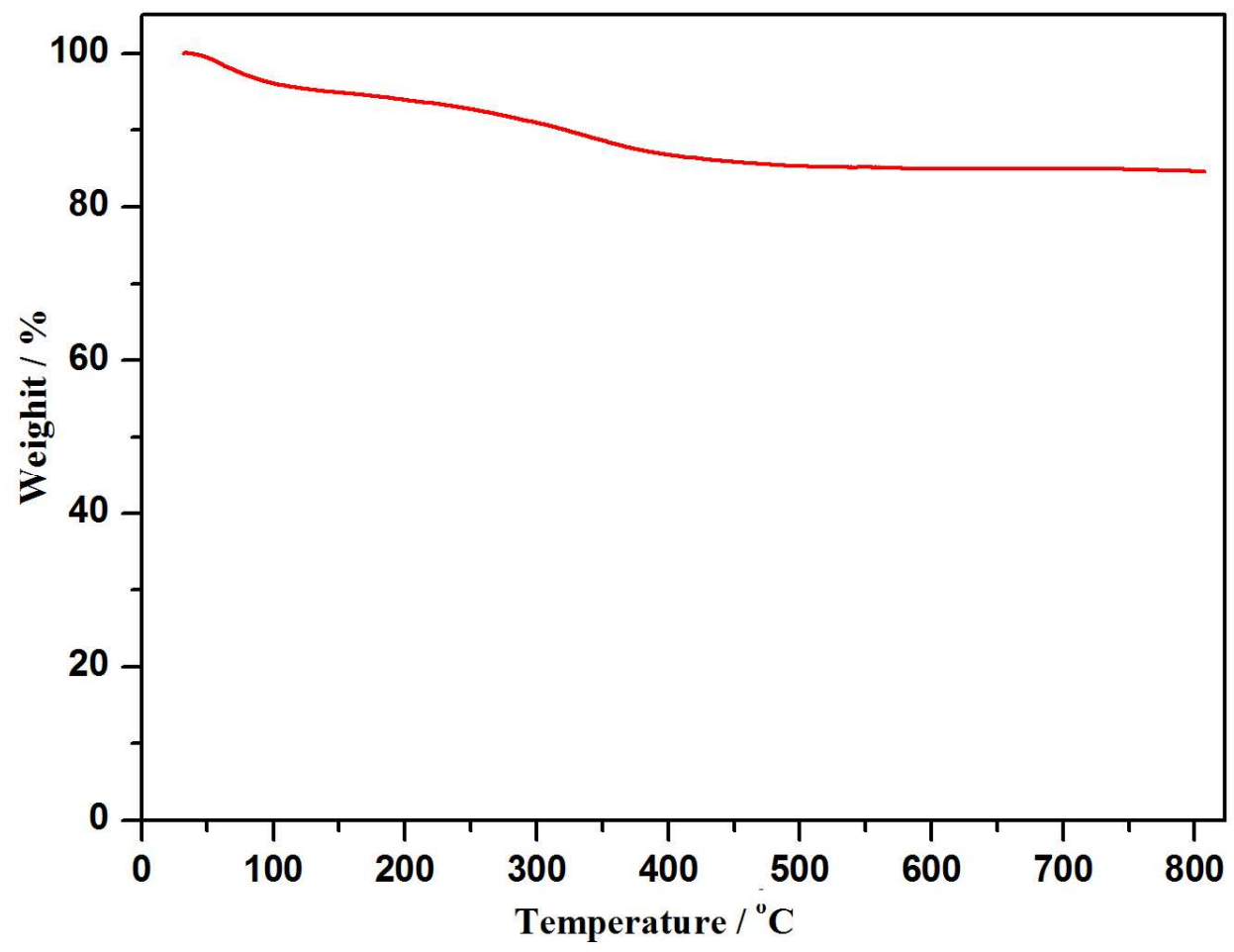

Figure S17. TGA curve for MnCo-1. 


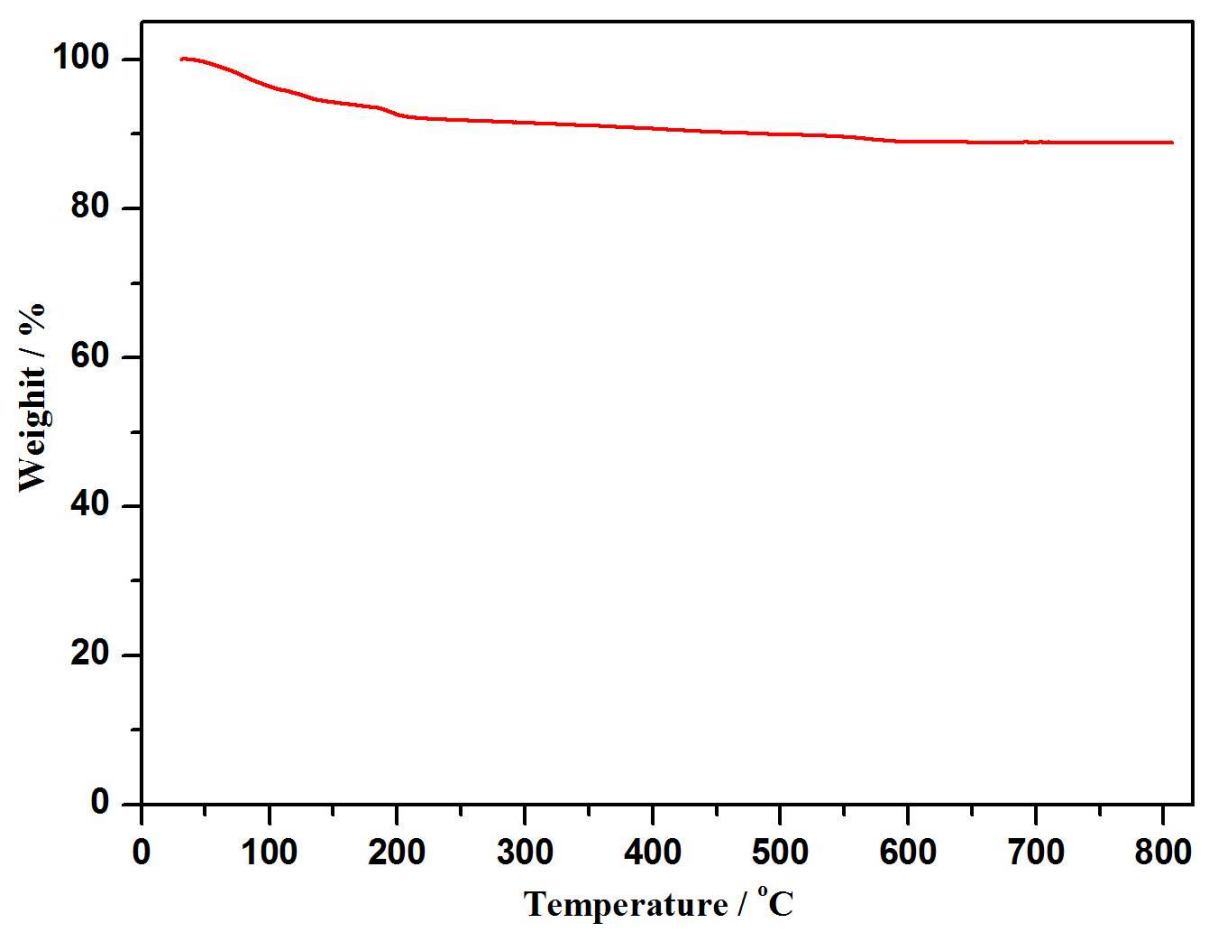

Figure S18. TGA curve for CoCo-1.

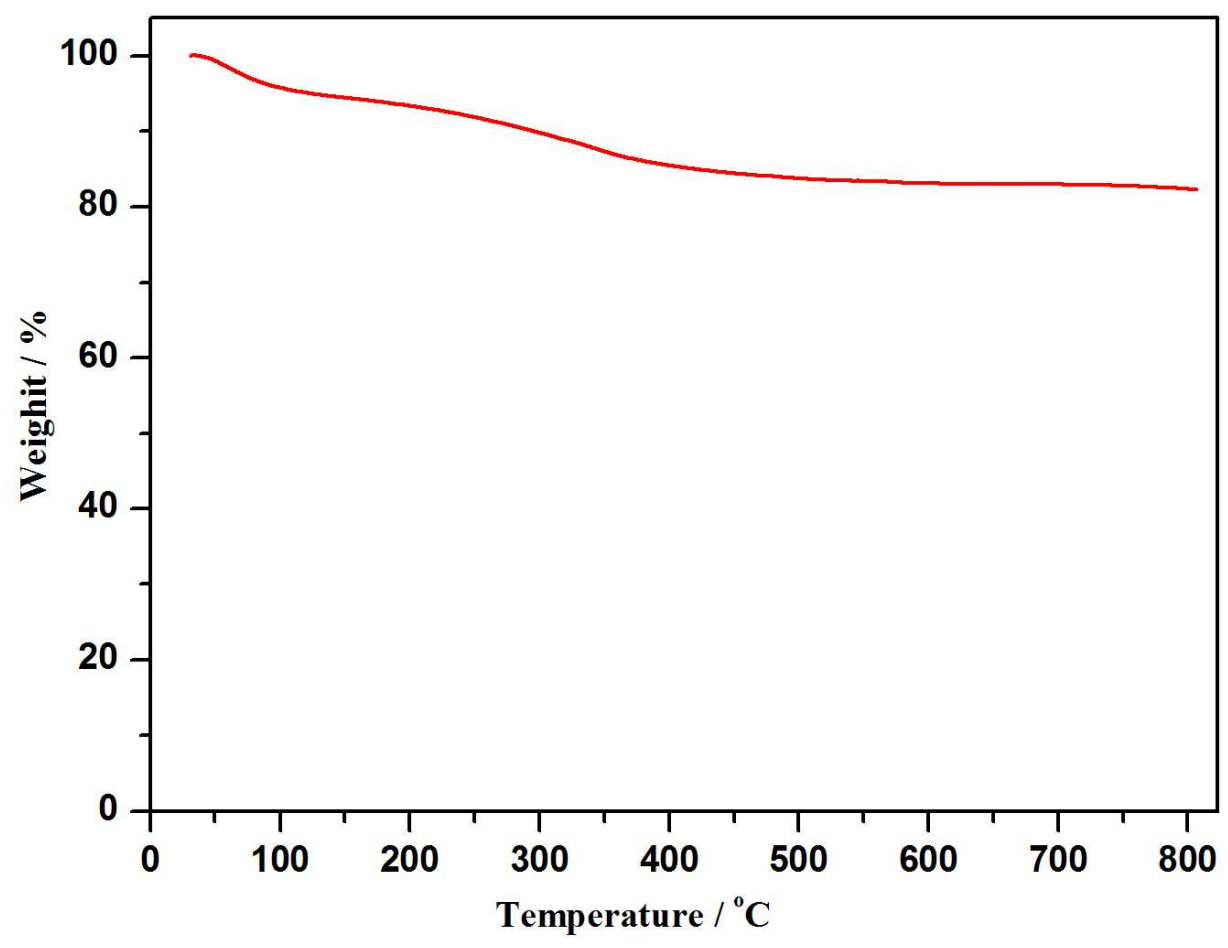

Figure S19. TGA curve for MnMn-1. 


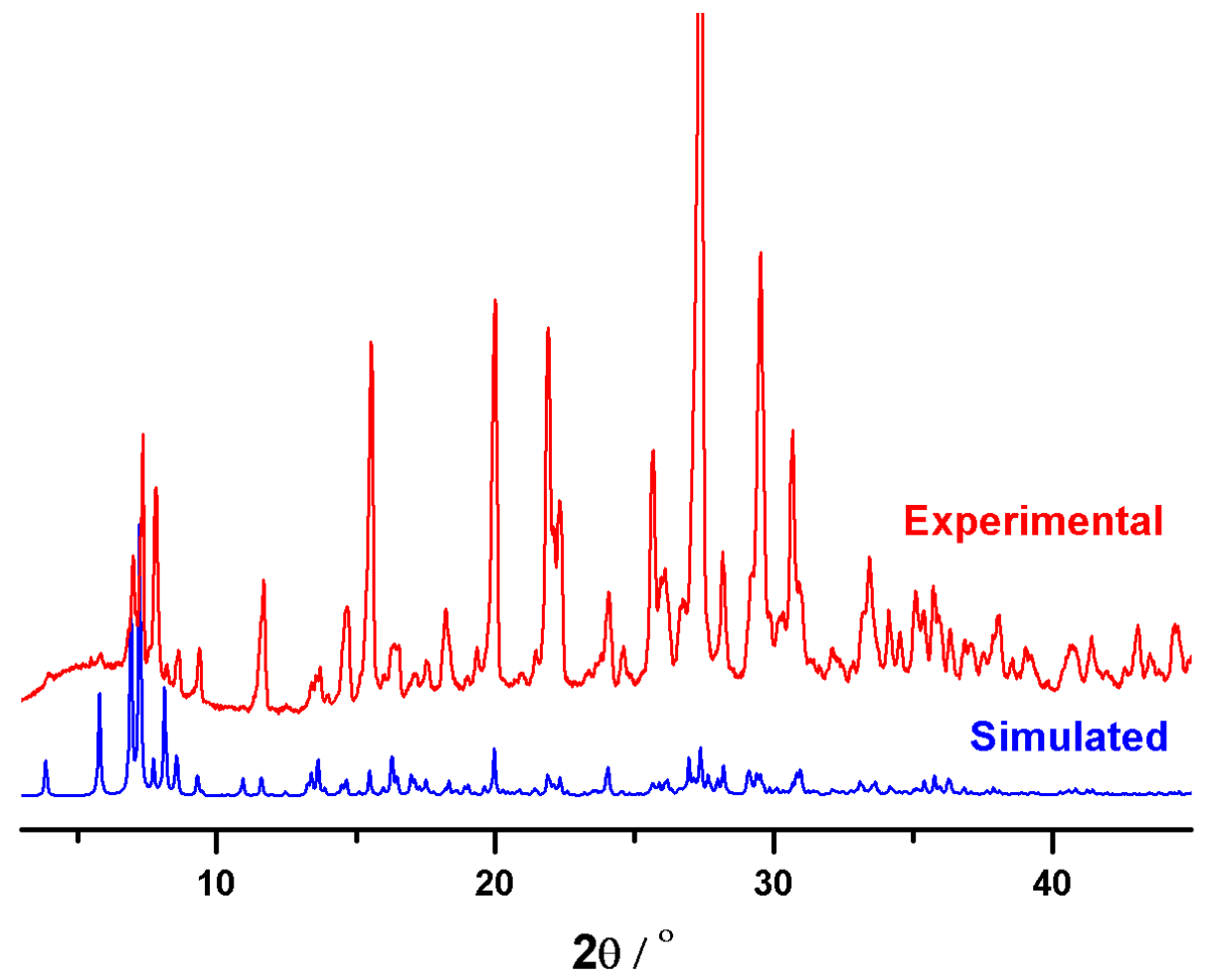

Figure S20. PXRD patterns for $\left\{\mathrm{Gd}_{4} \mathrm{Co}_{6} \mathrm{P}_{4} \mathrm{~W}_{30}\right\}$.

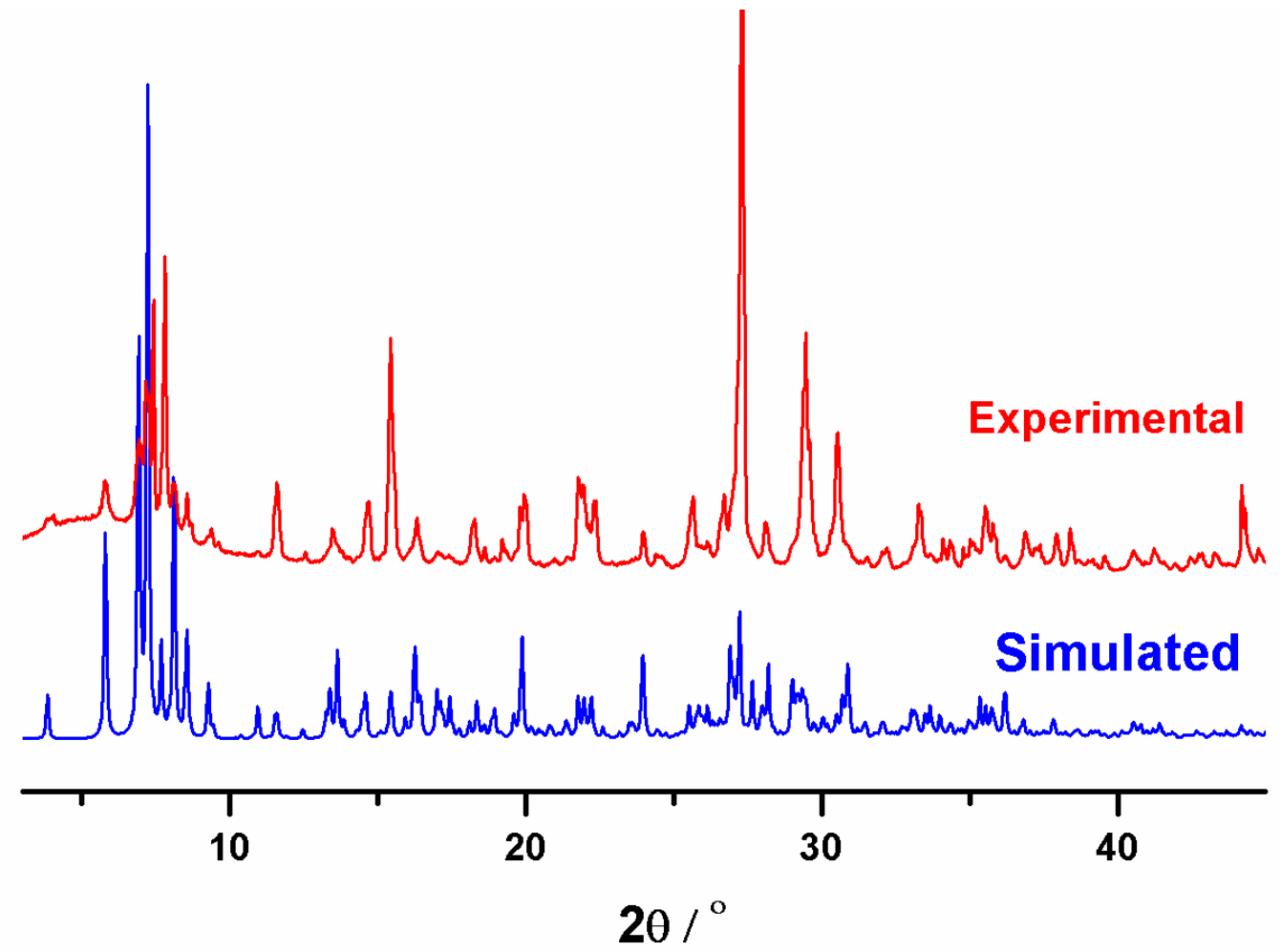

Figure S21. PXRD patterns for $\left\{\mathrm{Gd}_{4} \mathrm{Mn}_{6} \mathrm{P}_{4} \mathrm{~W}_{30}\right\}$. 


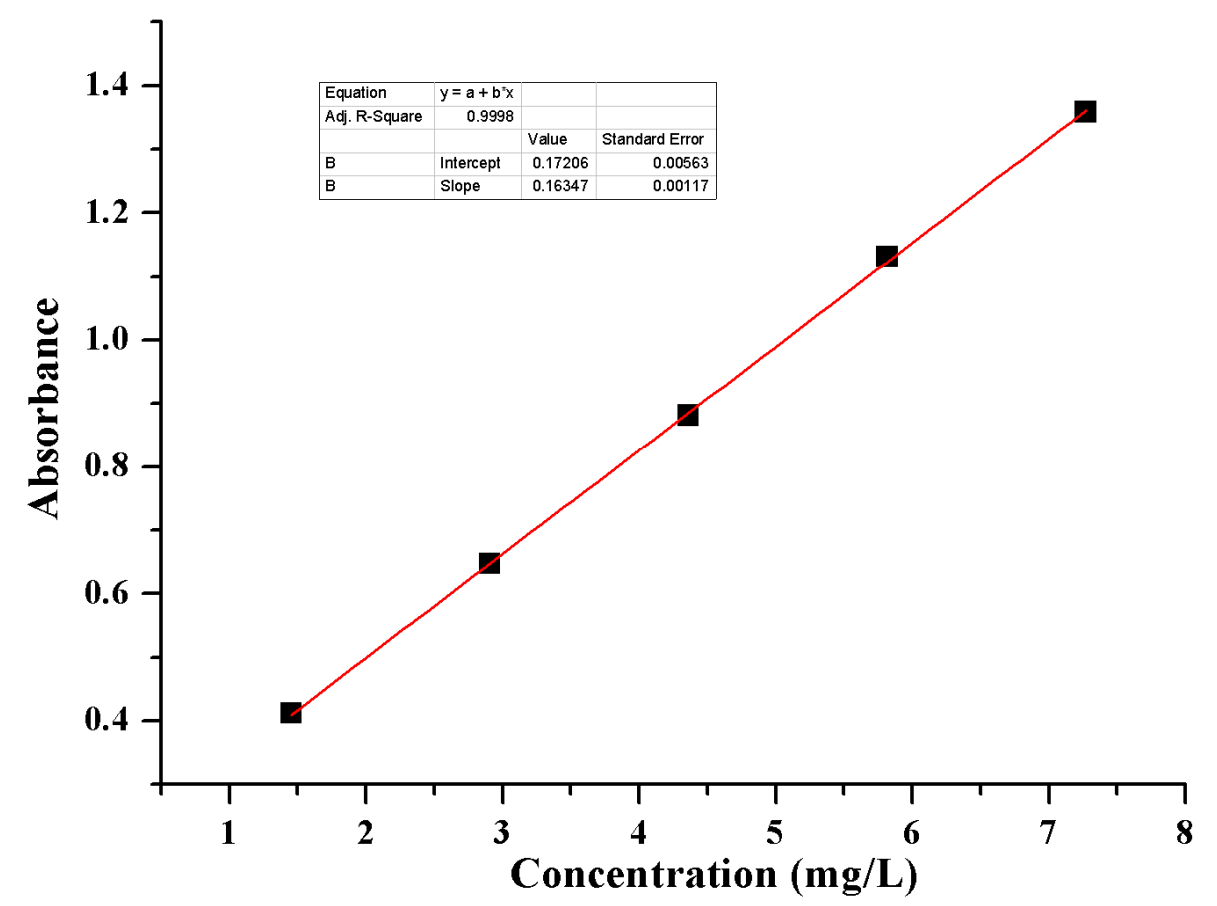

Figure S22. The relationship between the UV-Vis absorbance (monitored the sorption peak at $247 \mathrm{~nm}$ ) and styrene concentration in acetonitrile solvent.

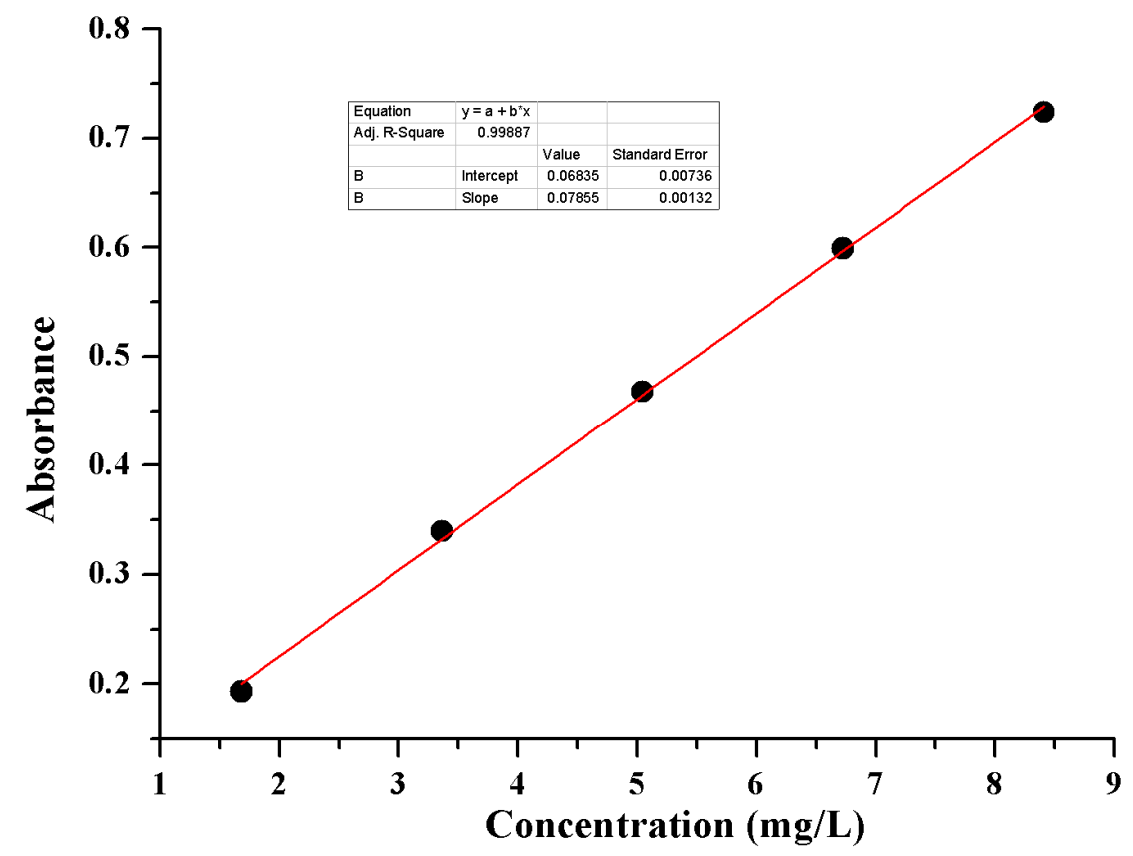

Figure S23. The relationship between the UV-Vis absorbance (monitored the sorption peak at $216 \mathrm{~nm}$ ) and 1,2-epoxyethylbenzene concentration in acetonitrile solvent. 


\section{Tables:}

Table S1. Crystal data and structural refinement for compound $\left\{\mathrm{Gd}_{4} \mathrm{M}_{6} \mathrm{P}_{4} \mathrm{~W}_{30}\right\}$

\begin{tabular}{|c|c|c|}
\hline Compound & $\left\{\mathrm{Gd}_{4} \mathrm{Co}_{6} \mathrm{P}_{4} \mathrm{~W}_{30}\right\}$ & $\left\{\mathrm{Gd}_{4} \mathrm{Mn}_{6} \mathrm{P}_{4} \mathrm{~W}_{30}\right\}$ \\
\hline Formula & $\mathrm{H}_{158} \mathrm{Co}_{6} \mathrm{Gd}_{4} \mathrm{O}_{191} \mathrm{P}_{4} \mathrm{~W}_{30}$ & $\mathrm{H}_{164} \mathrm{Gd}_{4} \mathrm{Mn}_{6} \mathrm{O}_{194} \mathrm{P}_{4} \mathrm{~W}_{30}$ \\
\hline Formula weight & 9837.22 & 9867.33 \\
\hline Crystal size/mm & $0.33 \times 0.21 \times 0.11$ & $0.21 \times 0.16 \times 0.11$ \\
\hline Crystal color & Pink & Brown \\
\hline Crystal system & Monoclinic & Monoclinic \\
\hline Space group & $C 2 / \mathrm{c}$ & $C 2 / \mathrm{c}$ \\
\hline$a(\AA)$ & $46.1852(18)$ & $46.4518(19)$ \\
\hline$b(\AA)$ & $16.1722(5)$ & $16.1574(5)$ \\
\hline$c(\AA)$ & $21.9613(7)$ & $22.0011(8)$ \\
\hline$\beta\left(^{\circ}\right)$ & $98.088(5)$ & $97.903(4)$ \\
\hline Volume $\left(\AA^{3}\right)$ & $16240.1(10)$ & $16355.9(10)$ \\
\hline Z & 4 & 4 \\
\hline$\rho\left(\mathrm{g} \cdot \mathrm{cm}^{-3}\right)$ & 4.023 & 4.007 \\
\hline$F(000)$ & 17536 & 17608 \\
\hline$\mu\left(\mathrm{mm}^{-1}\right)$ & 23.527 & 23.218 \\
\hline$\theta$ for data collection $\left({ }^{\circ}\right)$ & 2.87 to 25.06 & 2.87 to 25.06 \\
\hline Reflection collected & $52237\left(\mathrm{R}_{\mathrm{int}}=0.0949\right)$ & $57194\left(\mathrm{R}_{\mathrm{int}}=0.1163\right)$ \\
\hline Data / parameters & 14384 / 582 & 14477 / 595 \\
\hline Goodness-of fit on $F^{2}$ & 1.282 & 1.184 \\
\hline$R_{l}\left(\mathrm{w} R_{2}\right)[I>2 \sigma(I)]$ & $0.0885(0.2069)$ & $0.0735(0.1730)$ \\
\hline$R_{1}\left(\mathrm{w} R_{2}\right)$ (all data) & $0.1180(0.2226)$ & $0.0947(0.1890)$ \\
\hline
\end{tabular}


Table S2. Selected Bond lengths $[\AA]$ for $\left\{\mathrm{Gd}_{4} \mathrm{Co}_{6} \mathrm{P}_{4} \mathrm{~W}_{30}\right\}$.

\begin{tabular}{|c|c|c|c|c|c|}
\hline Bond lengths & (§) & Bond lengths & $(\AA)$ & Bond lengths & (§) \\
\hline $\mathrm{W}(1)-\mathrm{O}(39)$ & $1.722(19)$ & $\mathrm{W}(8)-\mathrm{O}(54)$ & $1.932(17)$ & $\mathrm{Gd}(1)-\mathrm{O}(70)$ & 2.41(3) \\
\hline $\mathrm{W}(1)-\mathrm{O}(46)$ & $1.771(19)$ & $\mathrm{W}(8)-\mathrm{O}(23)$ & $2.07(2)$ & $\mathrm{Gd}(1)-\mathrm{O}(76)$ & $2.42(3)$ \\
\hline $\mathrm{W}(1)-\mathrm{O}(15)$ & $1.901(16)$ & $\mathrm{W}(8)-\mathrm{O}(38)$ & $2.361(18)$ & $\mathrm{Gd}(1)-\mathrm{O}(2)$ & $2.43(2)$ \\
\hline $\mathrm{W}(1)-\mathrm{O}(52)$ & $1.918(15)$ & $\mathrm{W}(9)-\mathrm{O}(41)$ & $1.70(2)$ & $\mathrm{Gd}(1)-\mathrm{O}(68)$ & $2.43(4)$ \\
\hline $\mathrm{W}(1)-\mathrm{O}(51)$ & $2.03(2)$ & $\mathrm{W}(9)-\mathrm{O}(24)$ & $1.79(2)$ & $\mathrm{Gd}(1)-\mathrm{O}(69)$ & $2.44(3)$ \\
\hline $\mathrm{W}(1)-\mathrm{O}(12)$ & $2.346(17)$ & $\mathrm{W}(9)-\mathrm{O}(44)$ & $1.881(19)$ & $\mathrm{Gd}(1)-\mathrm{O}(60)$ & $2.455(17)$ \\
\hline $\mathrm{W}(2)-\mathrm{O}(30)$ & $1.70(2)$ & $\mathrm{W}(9)-\mathrm{O}(25)$ & $1.911(16)$ & $\mathrm{Gd}(1)-\mathrm{O}(6)$ & $2.46(2)$ \\
\hline $\mathrm{W}(2)-\mathrm{O}(64)$ & $1.77(2)$ & $\mathrm{W}(9)-\mathrm{O}(20)$ & $1.99(2)$ & $\mathrm{Gd}(1)-\mathrm{O}(71)$ & $2.46(2)$ \\
\hline $\mathrm{W}(2)-\mathrm{O}(54)$ & $1.882(18)$ & $\mathrm{W}(9)-\mathrm{O}(16)$ & $2.363(17)$ & $\mathrm{Gd}(1)-\mathrm{O}(67)$ & $2.47(4)$ \\
\hline $\mathrm{W}(2)-\mathrm{O}(61)$ & $1.968(17)$ & $\mathrm{W}(10)-\mathrm{O}(8)$ & $1.757(19)$ & $\mathrm{Gd}(2)-\mathrm{O}(35)$ & $2.40(2)$ \\
\hline $\mathrm{W}(2)-\mathrm{O}(24)$ & $2.07(2)$ & $\mathrm{W}(10)-\mathrm{O}(48)$ & $1.80(2)$ & $\mathrm{Gd}(2)-\mathrm{O}(7)$ & $2.41(2)$ \\
\hline $\mathrm{W}(2)-\mathrm{O}(18)$ & $2.302(17)$ & $\mathrm{W}(10)-\mathrm{O}(61)$ & $1.878(17)$ & $\mathrm{Gd}(2)-\mathrm{O}(39)$ & $2.42(2)$ \\
\hline $\mathrm{W}(3)-\mathrm{O}(40)$ & $1.727(18)$ & $\mathrm{W}(10)-\mathrm{O}(15)$ & $1.930(17)$ & $\mathrm{Gd}(2)-\mathrm{O}(13)$ & $2.45(2)$ \\
\hline $\mathrm{W}(3)-\mathrm{O}(37)$ & $1.78(2)$ & $\mathrm{W}(10)-\mathrm{O}(57)$ & $2.02(2)$ & $\mathrm{Gd}(2)-\mathrm{O}(66)$ & $2.45(2)$ \\
\hline $\mathrm{W}(3)-\mathrm{O}(32)$ & $1.888(18)$ & $\mathrm{W}(10)-\mathrm{O}(18)$ & $2.380(17)$ & $\mathrm{Gd}(2)-\mathrm{O}(78)$ & $2.46(3)$ \\
\hline $\mathrm{W}(3)-\mathrm{O}(50)$ & $1.943(17)$ & $\mathrm{W}(11)-\mathrm{O}(22)$ & $1.71(2)$ & $\mathrm{Gd}(2)-\mathrm{O}(26)$ & $2.476(18)$ \\
\hline $\mathrm{W}(3)-\mathrm{O}(47)$ & $2.06(2)$ & $\mathrm{W}(11)-\mathrm{O}(62)$ & $1.81(2)$ & $\mathrm{Gd}(2)-\mathrm{O}(34)$ & $2.495(19)$ \\
\hline $\mathrm{W}(3)-\mathrm{O}(38)$ & $2.353(17)$ & $\mathrm{W}(11)-\mathrm{O}(4)$ & $1.894(18)$ & $\mathrm{Gd}(2)-\mathrm{O}(11)$ & $2.50(2)$ \\
\hline $\mathrm{W}(4)-\mathrm{O}(60)$ & $1.743(17)$ & $\mathrm{W}(11)-\mathrm{O}(33)$ & $1.897(18)$ & $\mathrm{Co}(1)-\mathrm{O}(58)$ & $1.99(2)$ \\
\hline $\mathrm{W}(4)-\mathrm{O}(47)$ & $1.79(2)$ & $\mathrm{W}(11)-\mathrm{O}(56)$ & $2.03(2)$ & $\mathrm{Co}(1)-\mathrm{O}(37)$ & $2.037(19)$ \\
\hline $\mathrm{W}(4)-\mathrm{O}(4)$ & $1.909(19)$ & $\mathrm{W}(11)-\mathrm{O}(45)$ & $2.357(16)$ & $\mathrm{Co}(1)-\mathrm{O}(48)^{\mathrm{i}}$ & $2.051(19)$ \\
\hline $\mathrm{W}(4)-\mathrm{O}(55)$ & $1.911(16)$ & $\mathrm{W}(12)-\mathrm{O}(17)$ & $1.724(17)$ & $\mathrm{Co}(1)-\mathrm{O}(46)^{\mathrm{i}}$ & $2.095(18)$ \\
\hline $\mathrm{W}(4)-\mathrm{O}(28)$ & $1.97(2)$ & $\mathrm{W}(12)-\mathrm{O}(31)$ & $1.80(2)$ & $\mathrm{Co}(1)-\mathrm{O}(29)$ & $2.15(2)$ \\
\hline $\mathrm{W}(4)-\mathrm{O}(49)$ & $2.317(15)$ & $\mathrm{W}(12)-\mathrm{O}(52)$ & $1.908(16)$ & $\mathrm{Co}(1)-\mathrm{O}(65)$ & $2.265(19)$ \\
\hline $\mathrm{W}(5)-\mathrm{O}(21)$ & $1.729(19)$ & $\mathrm{W}(12)-\mathrm{O}(32)$ & $1.927(18)$ & $\mathrm{Co}(2)-\mathrm{O}(31)$ & $1.989(18)$ \\
\hline $\mathrm{W}(5)-\mathrm{O}(51)$ & $1.82(2)$ & $\mathrm{W}(12)-\mathrm{O}(62)$ & $2.05(2)$ & $\mathrm{Co}(2)-\mathrm{O}(64)^{\mathrm{i}}$ & $2.012(19)$ \\
\hline $\mathrm{W}(5)-\mathrm{O}(36)$ & $1.890(18)$ & $\mathrm{W}(12)-\mathrm{O}(12)$ & $2.334(16)$ & $\mathrm{Co}(2)-\mathrm{O}(65)^{\mathrm{i}}$ & $2.137(18)$ \\
\hline $\mathrm{W}(5)-\mathrm{O}(33)$ & $1.974(17)$ & $\mathrm{W}(13)-\mathrm{O}(9)$ & $1.70(2)$ & $\mathrm{Co}(2)-\mathrm{O}(65)$ & $2.15(2)$ \\
\hline $\mathrm{W}(5)-\mathrm{O}(59)$ & $2.008(19)$ & $\mathrm{W}(13)-\mathrm{O}(56)$ & $1.83(2)$ & $\mathrm{Co}(2)-\mathrm{O}(48)^{\mathrm{i}}$ & $2.156(19)$ \\
\hline $\mathrm{W}(5)-\mathrm{O}(45)$ & $2.351(17)$ & $\mathrm{W}(13)-\mathrm{O}(28)$ & $1.88(2)$ & $\mathrm{Co}(2)-\mathrm{O}(46)$ & $2.187(18)$ \\
\hline $\mathrm{W}(6)-\mathrm{O}(42)$ & $1.76(2)$ & $\mathrm{W}(13)-\mathrm{O}(27)$ & $1.922(19)$ & $\mathrm{Co}(3)-\mathrm{O}(72)$ & $2.01(3)$ \\
\hline $\mathrm{W}(6)-\mathrm{O}(23)$ & $1.80(2)$ & $\mathrm{W}(13)-\mathrm{O}(3)$ & $1.95(2)$ & $\mathrm{Co}(3)-\mathrm{O}(77)$ & $2.04(3)$ \\
\hline $\mathrm{W}(6)-\mathrm{O}(25)$ & $1.879(16)$ & $\mathrm{W}(13)-\mathrm{O}(63)$ & $2.383(16)$ & $\mathrm{Co}(3)-\mathrm{O}(73)$ & $2.05(3)$ \\
\hline $\mathrm{W}(6)-\mathrm{O}(55)$ & $1.976(15)$ & $\mathrm{W}(14)-\mathrm{O}(1)$ & $1.71(2)$ & $\mathrm{Co}(3)-\mathrm{O}(75)$ & $2.07(3)$ \\
\hline $\mathrm{W}(6)-\mathrm{O}(19)$ & $1.990(19)$ & $\mathrm{W}(14)-\mathrm{O}(19)$ & $1.878(18)$ & $\mathrm{Co}(3)-\mathrm{O}(42)$ & $2.15(2)$ \\
\hline $\mathrm{W}(6)-\mathrm{O}(49)$ & $2.342(17)$ & $\mathrm{W}(14)-\mathrm{O}(20)$ & $1.885(19)$ & $\mathrm{Co}(3)-\mathrm{O}(74)$ & $2.21(3)$ \\
\hline $\mathrm{W}(7)-\mathrm{O}(10)$ & $1.69(2)$ & $\mathrm{W}(14)-\mathrm{O}(27)$ & $1.932(19)$ & $\mathrm{P}(1)-\mathrm{O}(12)$ & $1.538(18)$ \\
\hline $\mathrm{W}(7)-\mathrm{O}(57)$ & $1.82(2)$ & $\mathrm{W}(14)-\mathrm{O}(14)$ & $1.97(2)$ & $\mathrm{P}(1)-\mathrm{O}(38)$ & $1.543(19)$ \\
\hline
\end{tabular}




\begin{tabular}{ll|ll|ll}
$\mathrm{W}(7)-\mathrm{O}(36)$ & $1.890(18)$ & $\mathrm{W}(14)-\mathrm{O}(63)$ & $2.341(18)$ & $\mathrm{P}(1)-\mathrm{O}(18)$ & $1.546(18)$ \\
$\mathrm{W}(7)-\mathrm{O}(44)$ & $1.95(2)$ & $\mathrm{W}(15)-\mathrm{O}(5)$ & $1.76(2)$ & $\mathrm{P}(1)-\mathrm{O}(65)$ & $1.55(2)$ \\
$\mathrm{W}(7)-\mathrm{O}(53)$ & $1.96(2)$ & $\mathrm{W}(15)-\mathrm{O}(59)$ & $1.851(19)$ & $\mathrm{P}(2)-\mathrm{O}(45)$ & $1.521(19)$ \\
$\mathrm{W}(7)-\mathrm{O}(16)$ & $2.370(17)$ & $\mathrm{W}(15)-\mathrm{O}(3)$ & $1.88(2)$ & $\mathrm{P}(2)-\mathrm{O}(16)$ & $1.538(18)$ \\
$\mathrm{W}(8)-\mathrm{O}(43)$ & $1.71(2)$ & $\mathrm{W}(15)-\mathrm{O}(53)$ & $1.901(19)$ & $\mathrm{P}(2)-\mathrm{O}(49)$ & $1.565(18)$ \\
$\mathrm{W}(8)-\mathrm{O}(58)$ & $1.81(2)$ & $\mathrm{W}(15)-\mathrm{O}(14)$ & $1.92(2)$ & $\mathrm{P}(2)-\mathrm{O}(63)$ & $1.60(2)$ \\
$\mathrm{W}(8)-\mathrm{O}(50)$ & $1.925(16)$ & $\mathrm{W}(15)-\mathrm{O}(63)$ & $2.363(17)$ & & \\
\hline
\end{tabular}

Symmetry transformations used to generate equivalent atoms: i) $-x+1 / 2,-y+1 / 2,-z$.

Table S3. Selected Bond Angles for $\left\{\mathrm{Gd}_{4} \mathrm{Co}_{6} \mathrm{P}_{4} \mathrm{~W}_{30}\right\}$.

\begin{tabular}{|c|c|c|c|c|c|}
\hline Bond angles & $\left({ }^{0}\right)$ & Bond angles & $\left({ }^{0}\right)$ & Bond angles & $\left({ }^{0}\right)$ \\
\hline $\mathrm{O}(39)-\mathrm{W}(1)-\mathrm{O}(46)$ & $99.5(9)$ & $\mathrm{O}(48)-\mathrm{W}(10)-\mathrm{O}(57)$ & $164.7(8)$ & $\mathrm{O}(39)-\mathrm{Gd}(2)-\mathrm{O}(34)$ & $134.2(7)$ \\
\hline $\mathrm{O}(39)-\mathrm{W}(1)-\mathrm{O}(15)$ & $100.7(8)$ & $\mathrm{O}(61)-\mathrm{W}(10)-\mathrm{O}(57)$ & $85.1(8)$ & $\mathrm{O}(13)-\mathrm{Gd}(2)-\mathrm{O}(34)$ & $69.4(6)$ \\
\hline $\mathrm{O}(46)-\mathrm{W}(1)-\mathrm{O}(15)$ & $93.0(8)$ & $\mathrm{O}(15)-\mathrm{W}(10)-\mathrm{O}(57)$ & $82.1(8)$ & $\mathrm{O}(66)-\mathrm{Gd}(2)-\mathrm{O}(34)$ & $140.9(7)$ \\
\hline $\mathrm{O}(39)-\mathrm{W}(1)-\mathrm{O}(52)$ & $101.5(8)$ & $\mathrm{O}(8)-\mathrm{W}(10)-\mathrm{O}(18)$ & $173.7(8)$ & $\mathrm{O}(78)-\mathrm{Gd}(2)-\mathrm{O}(34)$ & $114.4(8)$ \\
\hline $\mathrm{O}(46)-\mathrm{W}(1)-\mathrm{O}(52)$ & $95.6(8)$ & $\mathrm{O}(48)-\mathrm{W}(10)-\mathrm{O}(18)$ & $82.9(7)$ & $\mathrm{O}(26)-\mathrm{Gd}(2)-\mathrm{O}(34)$ & $71.1(6)$ \\
\hline $\mathrm{O}(15)-\mathrm{W}(1)-\mathrm{O}(52)$ & $154.4(7)$ & $\mathrm{O}(61)-\mathrm{W}(10)-\mathrm{O}(18)$ & $72.8(7)$ & $\mathrm{O}(35)-\mathrm{Gd}(2)-\mathrm{O}(11)$ & $71.4(7)$ \\
\hline $\mathrm{O}(39)-\mathrm{W}(1)-\mathrm{O}(51)$ & $93.5(9)$ & $\mathrm{O}(15)-\mathrm{W}(10)-\mathrm{O}(18)$ & $83.8(7)$ & $\mathrm{O}(7)-\mathrm{Gd}(2)-\mathrm{O}(11)$ & $130.6(7)$ \\
\hline $\mathrm{O}(46)-\mathrm{W}(1)-\mathrm{O}(51)$ & $166.9(8)$ & $\mathrm{O}(57)-\mathrm{W}(10)-\mathrm{O}(18)$ & $82.6(7)$ & $\mathrm{O}(39)-\mathrm{Gd}(2)-\mathrm{O}(11)$ & $71.0(7)$ \\
\hline $\mathrm{O}(15)-\mathrm{W}(1)-\mathrm{O}(51)$ & $82.6(8)$ & $\mathrm{O}(22)-\mathrm{W}(11)-\mathrm{O}(62)$ & $100.4(10)$ & $\mathrm{O}(13)-\mathrm{Gd}(2)-\mathrm{O}(11)$ & $130.8(7)$ \\
\hline $\mathrm{O}(52)-\mathrm{W}(1)-\mathrm{O}(51)$ & $83.6(7)$ & $\mathrm{O}(22)-\mathrm{W}(11)-\mathrm{O}(4)$ & $100.3(9)$ & $\mathrm{O}(66)-\mathrm{Gd}(2)-\mathrm{O}(11)$ & $65.4(7)$ \\
\hline $\mathrm{O}(39)-\mathrm{W}(1)-\mathrm{O}(12)$ & $173.9(7)$ & $\mathrm{O}(62)-\mathrm{W}(11)-\mathrm{O}(4)$ & $90.2(9)$ & $\mathrm{O}(78)-\mathrm{Gd}(2)-\mathrm{O}(11)$ & $122.7(8)$ \\
\hline $\mathrm{O}(46)-\mathrm{W}(1)-\mathrm{O}(12)$ & $84.0(7)$ & $\mathrm{O}(22)-\mathrm{W}(11)-\mathrm{O}(33)$ & $98.6(9)$ & $\mathrm{O}(26)-\mathrm{Gd}(2)-\mathrm{O}(11)$ & $67.1(6)$ \\
\hline $\mathrm{O}(15)-\mathrm{W}(1)-\mathrm{O}(12)$ & $84.1(7)$ & $\mathrm{O}(62)-\mathrm{W}(11)-\mathrm{O}(33)$ & $92.5(9)$ & $\mathrm{O}(34)-\mathrm{Gd}(2)-\mathrm{O}(11)$ & $122.9(6)$ \\
\hline $\mathrm{O}(52)-\mathrm{W}(1)-\mathrm{O}(12)$ & $73.0(6)$ & $\mathrm{O}(4)-\mathrm{W}(11)-\mathrm{O}(33)$ & $160.1(8)$ & $\mathrm{O}(58)-\mathrm{Co}(1)-\mathrm{O}(37)$ & $93.8(8)$ \\
\hline $\mathrm{O}(51)-\mathrm{W}(1)-\mathrm{O}(12)$ & $83.3(7)$ & $\mathrm{O}(22)-\mathrm{W}(11)-\mathrm{O}(56)$ & $97.1(10)$ & $\mathrm{O}(58)-\mathrm{Co}(1)-\mathrm{O}(48)^{\mathrm{i}}$ & $170.9(7)$ \\
\hline $\mathrm{O}(30)-\mathrm{W}(2)-\mathrm{O}(64)$ & $101.5(10)$ & $\mathrm{O}(62)-\mathrm{W}(11)-\mathrm{O}(56)$ & $162.4(8)$ & $\mathrm{O}(37)-\mathrm{Co}(1)-\mathrm{O}(48)^{\mathrm{i}}$ & $93.3(8)$ \\
\hline $\mathrm{O}(30)-\mathrm{W}(2)-\mathrm{O}(54)$ & $101.5(9)$ & $\mathrm{O}(4)-\mathrm{W}(11)-\mathrm{O}(56)$ & $84.7(8)$ & $\mathrm{O}(58)-\mathrm{Co}(1)-\mathrm{O}(46)^{\mathrm{i}}$ & $89.2(7)$ \\
\hline $\mathrm{O}(64)-\mathrm{W}(2)-\mathrm{O}(54)$ & $96.4(8)$ & $\mathrm{O}(33)-\mathrm{W}(11)-\mathrm{O}(56)$ & $86.8(8)$ & $\mathrm{O}(37)-\mathrm{Co}(1)-\mathrm{O}(46)^{\mathrm{i}}$ & $175.1(8)$ \\
\hline $\mathrm{O}(30)-\mathrm{W}(2)-\mathrm{O}(61)$ & $100.2(8)$ & $\mathrm{O}(22)-\mathrm{W}(11)-\mathrm{O}(45)$ & $172.6(9)$ & $\mathrm{O}(48)^{\mathrm{i}}-\mathrm{Co}(1)-\mathrm{O}(46)^{\mathrm{i}}$ & $83.3(7)$ \\
\hline $\mathrm{O}(64)-\mathrm{W}(2)-\mathrm{O}(61)$ & $91.5(8)$ & $\mathrm{O}(62)-\mathrm{W}(11)-\mathrm{O}(45)$ & $83.0(7)$ & $\mathrm{O}(58)-\mathrm{Co}(1)-\mathrm{O}(29)$ & $89.9(8)$ \\
\hline $\mathrm{O}(54)-\mathrm{W}(2)-\mathrm{O}(61)$ & $154.9(8)$ & $\mathrm{O}(4)-\mathrm{W}(11)-\mathrm{O}(45)$ & $86.2(7)$ & $\mathrm{O}(37)-\mathrm{Co}(1)-\mathrm{O}(29)$ & $89.4(7)$ \\
\hline $\mathrm{O}(30)-\mathrm{W}(2)-\mathrm{O}(24)$ & $92.7(9)$ & $\mathrm{O}(33)-\mathrm{W}(11)-\mathrm{O}(45)$ & $74.6(7)$ & $\mathrm{O}(48)^{\mathrm{i}}-\mathrm{Co}(1)-\mathrm{O}(29)$ & $95.9(8)$ \\
\hline $\mathrm{O}(64)-\mathrm{W}(2)-\mathrm{O}(24)$ & $165.4(8)$ & $\mathrm{O}(56)-\mathrm{W}(11)-\mathrm{O}(45)$ & $79.8(7)$ & $\mathrm{O}(46)^{\mathrm{i}}-\mathrm{Co}(1)-\mathrm{O}(29)$ & $94.5(7)$ \\
\hline $\mathrm{O}(54)-\mathrm{W}(2)-\mathrm{O}(24)$ & $84.2(8)$ & $\mathrm{O}(17)-\mathrm{W}(12)-\mathrm{O}(31)$ & $101.0(9)$ & $\mathrm{O}(58)-\mathrm{Co}(1)-\mathrm{O}(65)$ & $88.8(8)$ \\
\hline $\mathrm{O}(61)-\mathrm{W}(2)-\mathrm{O}(24)$ & $82.3(8)$ & $\mathrm{O}(17)-\mathrm{W}(12)-\mathrm{O}(52)$ & $100.0(8)$ & $\mathrm{O}(37)-\mathrm{Co}(1)-\mathrm{O}(65)$ & $92.9(7)$ \\
\hline
\end{tabular}




\begin{tabular}{|c|c|c|c|c|c|}
\hline $\mathrm{O}(30)-\mathrm{W}(2)-\mathrm{O}(18)$ & $171.6(8)$ & $\mathrm{O}(31)-\mathrm{W}(12)-\mathrm{O}(52)$ & $92.4(8)$ & $\mathrm{O}(48)^{\mathrm{i}}-\mathrm{Co}(1)-\mathrm{O}(65)$ & $85.1(7)$ \\
\hline $\mathrm{O}(64)-\mathrm{W}(2)-\mathrm{O}(18)$ & $84.1(8)$ & $\mathrm{O}(17)-\mathrm{W}(12)-\mathrm{O}(32)$ & $102.1(8)$ & $\mathrm{O}(46)^{\mathrm{i}}-\mathrm{Co}(1)-\mathrm{O}(65)$ & $83.3(7)$ \\
\hline $\mathrm{O}(54)-\mathrm{W}(2)-\mathrm{O}(18)$ & $84.0(7)$ & $\mathrm{O}(31)-\mathrm{W}(12)-\mathrm{O}(32)$ & $96.2(8)$ & $\mathrm{O}(29)-\mathrm{Co}(1)-\mathrm{O}(65)$ & $177.5(7)$ \\
\hline $\mathrm{O}(61)-\mathrm{W}(2)-\mathrm{O}(18)$ & $73.1(6)$ & $\mathrm{O}(52)-\mathrm{W}(12)-\mathrm{O}(32)$ & $154.3(7)$ & $\mathrm{O}(31)-\mathrm{Co}(2)-\mathrm{O}(64)^{\mathrm{i}}$ & $92.0(8)$ \\
\hline $\mathrm{O}(24)-\mathrm{W}(2)-\mathrm{O}(18)$ & $81.5(7)$ & $\mathrm{O}(17)-\mathrm{W}(12)-\mathrm{O}(62)$ & $93.8(8)$ & $\mathrm{O}(31)-\mathrm{Co}(2)-\mathrm{O}(65)^{\mathrm{i}}$ & $175.8(8)$ \\
\hline $\mathrm{O}(40)-\mathrm{W}(3)-\mathrm{O}(37)$ & $102.6(9)$ & $\mathrm{O}(31)-\mathrm{W}(12)-\mathrm{O}(62)$ & $165.0(7)$ & $\mathrm{O}(64)^{\mathrm{i}}-\mathrm{Co}(2)-\mathrm{O}(65)^{\mathrm{i}}$ & $92.2(8)$ \\
\hline $\mathrm{O}(40)-\mathrm{W}(3)-\mathrm{O}(32)$ & $100.4(8)$ & $\mathrm{O}(52)-\mathrm{W}(12)-\mathrm{O}(62)$ & $82.9(8)$ & $\mathrm{O}(31)-\mathrm{Co}(2)-\mathrm{O}(65)$ & $91.3(8)$ \\
\hline $\mathrm{O}(37)-\mathrm{W}(3)-\mathrm{O}(32)$ & $98.0(8)$ & $\mathrm{O}(32)-\mathrm{W}(12)-\mathrm{O}(62)$ & $82.6(8)$ & $\mathrm{O}(64)^{\mathrm{i}}-\mathrm{Co}(2)-\mathrm{O}(65)$ & $176.6(7)$ \\
\hline $\mathrm{O}(40)-\mathrm{W}(3)-\mathrm{O}(50)$ & $100.2(8)$ & $\mathrm{O}(17)-\mathrm{W}(12)-\mathrm{O}(12)$ & $172.4(7)$ & $\mathrm{O}(65)^{\mathrm{i}}-\mathrm{Co}(2)-\mathrm{O}(65)$ & $84.5(8)$ \\
\hline $\mathrm{O}(37)-\mathrm{W}(3)-\mathrm{O}(50)$ & $92.8(8)$ & $\mathrm{O}(31)-\mathrm{W}(12)-\mathrm{O}(12)$ & $83.3(7)$ & $\mathrm{O}(31)-\mathrm{Co}(2)-\mathrm{O}(48)^{\mathrm{i}}$ & $91.4(7)$ \\
\hline $\mathrm{O}(32)-\mathrm{W}(3)-\mathrm{O}(50)$ & $154.0(7)$ & $\mathrm{O}(52)-\mathrm{W}(12)-\mathrm{O}(12)$ & $73.4(6)$ & $\mathrm{O}(64)^{\mathrm{i}}-\mathrm{Co}(2)-\mathrm{O}(48)^{\mathrm{i}}$ & $95.5(7)$ \\
\hline $\mathrm{O}(40)-\mathrm{W}(3)-\mathrm{O}(47)$ & $91.4(8)$ & $\mathrm{O}(32)-\mathrm{W}(12)-\mathrm{O}(12)$ & $83.6(7)$ & $\mathrm{O}(65)^{\mathrm{i}}-\mathrm{Co}(2)-\mathrm{O}(48)^{\mathrm{i}}$ & $87.7(7)$ \\
\hline $\mathrm{O}(37)-\mathrm{W}(3)-\mathrm{O}(47)$ & $165.3(7)$ & $\mathrm{O}(62)-\mathrm{W}(12)-\mathrm{O}(12)$ & $81.8(7)$ & $\mathrm{O}(65)-\mathrm{Co}(2)-\mathrm{O}(48)^{\mathrm{i}}$ & $85.4(7)$ \\
\hline $\mathrm{O}(32)-\mathrm{W}(3)-\mathrm{O}(47)$ & $83.5(8)$ & $\mathrm{O}(9)-\mathrm{W}(13)-\mathrm{O}(56)$ & $101.4(10)$ & $\mathrm{O}(31)-\mathrm{Co}(2)-\mathrm{O}(46)$ & $96.1(7)$ \\
\hline $\mathrm{O}(50)-\mathrm{W}(3)-\mathrm{O}(47)$ & $80.3(7)$ & $\mathrm{O}(9)-\mathrm{W}(13)-\mathrm{O}(28)$ & $102.4(10)$ & $\mathrm{O}(64)^{\mathrm{i}}-\mathrm{Co}(2)-\mathrm{O}(46)$ & $92.3(7)$ \\
\hline $\mathrm{O}(40)-\mathrm{W}(3)-\mathrm{O}(38)$ & $170.7(8)$ & $\mathrm{O}(56)-\mathrm{W}(13)-\mathrm{O}(28)$ & $90.0(9)$ & $\mathrm{O}(65)^{\mathrm{i}}-\mathrm{Co}(2)-\mathrm{O}(46)$ & $84.3(7)$ \\
\hline $\mathrm{O}(37)-\mathrm{W}(3)-\mathrm{O}(38)$ & $85.4(7)$ & $\mathrm{O}(9)-\mathrm{W}(13)-\mathrm{O}(27)$ & $102.9(10)$ & $\mathrm{O}(65)-\mathrm{Co}(2)-\mathrm{O}(46)$ & $86.4(7)$ \\
\hline $\mathrm{O}(32)-\mathrm{W}(3)-\mathrm{O}(38)$ & $83.0(7)$ & $\mathrm{O}(56)-\mathrm{W}(13)-\mathrm{O}(27)$ & $155.6(9)$ & $\mathrm{O}(48)^{\mathrm{i}}-\mathrm{Co}(2)-\mathrm{O}(46)$ & $169.0(7)$ \\
\hline $\mathrm{O}(50)-\mathrm{W}(3)-\mathrm{O}(38)$ & $74.4(6)$ & $\mathrm{O}(28)-\mathrm{W}(13)-\mathrm{O}(27)$ & $87.3(9)$ & $\mathrm{O}(72)-\mathrm{Co}(3)-\mathrm{O}(77)$ & $173.9(12)$ \\
\hline $\mathrm{O}(47)-\mathrm{W}(3)-\mathrm{O}(38)$ & $80.3(7)$ & $\mathrm{O}(9)-\mathrm{W}(13)-\mathrm{O}(3)$ & $102.8(10)$ & $\mathrm{O}(72)-\mathrm{Co}(3)-\mathrm{O}(73)$ & $87.8(13)$ \\
\hline $\mathrm{O}(60)-\mathrm{W}(4)-\mathrm{O}(47)$ & 101.2(8) & $\mathrm{O}(56)-\mathrm{W}(13)-\mathrm{O}(3)$ & $83.1(9)$ & $\mathrm{O}(77)-\mathrm{Co}(3)-\mathrm{O}(73)$ & $87.6(12)$ \\
\hline $\mathrm{O}(60)-\mathrm{W}(4)-\mathrm{O}(4)$ & $100.8(8)$ & $\mathrm{O}(28)-\mathrm{W}(13)-\mathrm{O}(3)$ & 154.7(9) & $\mathrm{O}(72)-\mathrm{Co}(3)-\mathrm{O}(75)$ & $94.6(12)$ \\
\hline $\mathrm{O}(47)-\mathrm{W}(4)-\mathrm{O}(4)$ & $89.6(8)$ & $\mathrm{O}(27)-\mathrm{W}(13)-\mathrm{O}(3)$ & $89.1(9)$ & $\mathrm{O}(77)-\mathrm{Co}(3)-\mathrm{O}(75)$ & $89.8(11)$ \\
\hline $\mathrm{O}(60)-\mathrm{W}(4)-\mathrm{O}(55)$ & $97.3(8)$ & $\mathrm{O}(9)-\mathrm{W}(13)-\mathrm{O}(63)$ & $170.5(9)$ & $\mathrm{O}(73)-\mathrm{Co}(3)-\mathrm{O}(75)$ & $176.5(13)$ \\
\hline $\mathrm{O}(47)-\mathrm{W}(4)-\mathrm{O}(55)$ & $93.5(8)$ & $\mathrm{O}(56)-\mathrm{W}(13)-\mathrm{O}(63)$ & $84.5(8)$ & $\mathrm{O}(72)-\mathrm{Co}(3)-\mathrm{O}(42)$ & $92.2(10)$ \\
\hline $\mathrm{O}(4)-\mathrm{W}(4)-\mathrm{O}(55)$ & $160.7(7)$ & $\mathrm{O}(28)-\mathrm{W}(13)-\mathrm{O}(63)$ & $84.9(7)$ & $\mathrm{O}(77)-\mathrm{Co}(3)-\mathrm{O}(42)$ & $91.8(10)$ \\
\hline $\mathrm{O}(60)-\mathrm{W}(4)-\mathrm{O}(28)$ & 95.2(9) & $\mathrm{O}(27)-\mathrm{W}(13)-\mathrm{O}(63)$ & $71.1(7)$ & $\mathrm{O}(73)-\mathrm{Co}(3)-\mathrm{O}(42)$ & $90.9(10)$ \\
\hline $\mathrm{O}(47)-\mathrm{W}(4)-\mathrm{O}(28)$ & $163.6(8)$ & $\mathrm{O}(3)-\mathrm{W}(13)-\mathrm{O}(63)$ & $70.2(8)$ & $\mathrm{O}(75)-\mathrm{Co}(3)-\mathrm{O}(42)$ & $91.6(9)$ \\
\hline $\mathrm{O}(4)-\mathrm{W}(4)-\mathrm{O}(28)$ & $85.8(9)$ & $\mathrm{O}(1)-\mathrm{W}(14)-\mathrm{O}(19)$ & $102.7(9)$ & $\mathrm{O}(72)-\mathrm{Co}(3)-\mathrm{O}(74)$ & $89.1(12)$ \\
\hline $\mathrm{O}(55)-\mathrm{W}(4)-\mathrm{O}(28)$ & $85.9(8)$ & $\mathrm{O}(1)-\mathrm{W}(14)-\mathrm{O}(20)$ & 104.2(9) & $\mathrm{O}(77)-\mathrm{Co}(3)-\mathrm{O}(74)$ & $86.9(11)$ \\
\hline $\mathrm{O}(60)-\mathrm{W}(4)-\mathrm{O}(49)$ & $171.6(8)$ & $\mathrm{O}(19)-\mathrm{W}(14)-\mathrm{O}(20)$ & $89.9(8)$ & $\mathrm{O}(73)-\mathrm{Co}(3)-\mathrm{O}(74)$ & $89.9(11)$ \\
\hline $\mathrm{O}(47)-\mathrm{W}(4)-\mathrm{O}(49)$ & $82.2(7)$ & $\mathrm{O}(1)-\mathrm{W}(14)-\mathrm{O}(27)$ & $99.0(9)$ & $\mathrm{O}(75)-\mathrm{Co}(3)-\mathrm{O}(74)$ & $87.6(10)$ \\
\hline $\mathrm{O}(4)-\mathrm{W}(4)-\mathrm{O}(49)$ & $86.8(7)$ & $\mathrm{O}(19)-\mathrm{W}(14)-\mathrm{O}(27)$ & $87.8(8)$ & $\mathrm{O}(42)-\mathrm{Co}(3)-\mathrm{O}(74)$ & $178.5(10)$ \\
\hline $\mathrm{O}(55)-\mathrm{W}(4)-\mathrm{O}(49)$ & $74.8(6)$ & $\mathrm{O}(20)-\mathrm{W}(14)-\mathrm{O}(27)$ & $156.5(9)$ & $\mathrm{O}(12)-\mathrm{P}(1)-\mathrm{O}(38)$ & $109.4(10)$ \\
\hline $\mathrm{O}(28)-\mathrm{W}(4)-\mathrm{O}(49)$ & $81.8(7)$ & $\mathrm{O}(1)-\mathrm{W}(14)-\mathrm{O}(14)$ & $99.4(10)$ & $\mathrm{O}(12)-\mathrm{P}(1)-\mathrm{O}(18)$ & $111.8(10)$ \\
\hline $\mathrm{O}(21)-\mathrm{W}(5)-\mathrm{O}(51)$ & $100.3(9)$ & $\mathrm{O}(19)-\mathrm{W}(14)-\mathrm{O}(14)$ & $157.7(8)$ & $\mathrm{O}(38)-\mathrm{P}(1)-\mathrm{O}(18)$ & $109.9(10)$ \\
\hline $\mathrm{O}(21)-\mathrm{W}(5)-\mathrm{O}(36)$ & $101.6(8)$ & $\mathrm{O}(20)-\mathrm{W}(14)-\mathrm{O}(14)$ & $87.3(8)$ & $\mathrm{O}(12)-\mathrm{P}(1)-\mathrm{O}(65)$ & $107.5(10)$ \\
\hline $\mathrm{O}(51)-\mathrm{W}(5)-\mathrm{O}(36)$ & $90.9(8)$ & $\mathrm{O}(27)-\mathrm{W}(14)-\mathrm{O}(14)$ & $86.1(8)$ & $\mathrm{O}(38)-\mathrm{P}(1)-\mathrm{O}(65)$ & $109.8(11)$ \\
\hline $\mathrm{O}(21)-\mathrm{W}(5)-\mathrm{O}(33)$ & $99.9(8)$ & $\mathrm{O}(1)-\mathrm{W}(14)-\mathrm{O}(63)$ & $167.9(9)$ & $\mathrm{O}(18)-\mathrm{P}(1)-\mathrm{O}(65)$ & $108.5(10)$ \\
\hline $\mathrm{O}(51)-\mathrm{W}(5)-\mathrm{O}(33)$ & $92.3(8)$ & $\mathrm{O}(19)-\mathrm{W}(14)-\mathrm{O}(63)$ & $85.4(7)$ & $\mathrm{O}(45)-\mathrm{P}(2)-\mathrm{O}(16)$ & $111.5(10)$ \\
\hline $\mathrm{O}(36)-\mathrm{W}(5)-\mathrm{O}(33)$ & $157.3(8)$ & $\mathrm{O}(20)-\mathrm{W}(14)-\mathrm{O}(63)$ & $84.6(7)$ & $\mathrm{O}(45)-\mathrm{P}(2)-\mathrm{O}(49)$ & $114.1(10)$ \\
\hline
\end{tabular}




\begin{tabular}{|c|c|c|c|c|c|}
\hline $\mathrm{O}(21)-\mathrm{W}(5)-\mathrm{O}(59)$ & $96.8(9)$ & $\mathrm{O}(27)-\mathrm{W}(14)-\mathrm{O}(63)$ & $71.9(7)$ & $\mathrm{O}(16)-\mathrm{P}(2)-\mathrm{O}(49)$ & $111.0(10)$ \\
\hline $\mathrm{O}(51)-\mathrm{W}(5)-\mathrm{O}(59)$ & $162.7(8)$ & $\mathrm{O}(14)-\mathrm{W}(14)-\mathrm{O}(63)$ & $72.4(7)$ & $\mathrm{O}(45)-\mathrm{P}(2)-\mathrm{O}(63)$ & $106.7(10)$ \\
\hline $\mathrm{O}(36)-\mathrm{W}(5)-\mathrm{O}(59)$ & $83.4(8)$ & $\mathrm{O}(5)-\mathrm{W}(15)-\mathrm{O}(59)$ & $102.7(9)$ & $\mathrm{O}(16)-\mathrm{P}(2)-\mathrm{O}(63)$ & $107.4(10)$ \\
\hline $\mathrm{O}(33)-\mathrm{W}(5)-\mathrm{O}(59)$ & $87.0(7)$ & $\mathrm{O}(5)-\mathrm{W}(15)-\mathrm{O}(3)$ & $101.8(10)$ & $\mathrm{O}(49)-\mathrm{P}(2)-\mathrm{O}(63)$ & $105.6(10)$ \\
\hline $\mathrm{O}(21)-\mathrm{W}(5)-\mathrm{O}(45)$ & $172.8(8)$ & $\mathrm{O}(59)-\mathrm{W}(15)-\mathrm{O}(3)$ & 84.3(9) & $\mathrm{W}(15)-\mathrm{O}(3)-\mathrm{W}(13)$ & $125.8(12)$ \\
\hline $\mathrm{O}(51)-\mathrm{W}(5)-\mathrm{O}(45)$ & $83.0(8)$ & $\mathrm{O}(5)-\mathrm{W}(15)-\mathrm{O}(53)$ & $103.5(9)$ & $\mathrm{W}(11)-\mathrm{O}(4)-\mathrm{W}(4)$ & $152.3(11)$ \\
\hline $\mathrm{O}(36)-\mathrm{W}(5)-\mathrm{O}(45)$ & $84.7(7)$ & $\mathrm{O}(59)-\mathrm{W}(15)-\mathrm{O}(53)$ & $88.5(8)$ & $\mathrm{P}(1)-\mathrm{O}(12)-\mathrm{W}(12)$ & $127.6(10)$ \\
\hline $\mathrm{O}(33)-\mathrm{W}(5)-\mathrm{O}(45)$ & $73.5(7)$ & $\mathrm{O}(3)-\mathrm{W}(15)-\mathrm{O}(53)$ & $154.7(9)$ & $\mathrm{P}(1)-\mathrm{O}(12)-\mathrm{W}(1)$ & 127.1(9) \\
\hline $\mathrm{O}(59)-\mathrm{W}(5)-\mathrm{O}(45)$ & $80.2(7)$ & $\mathrm{O}(5)-\mathrm{W}(15)-\mathrm{O}(14)$ & $99.2(10)$ & $\mathrm{W}(12)-\mathrm{O}(12)-\mathrm{W}(1)$ & $91.2(6)$ \\
\hline $\mathrm{O}(42)-\mathrm{W}(6)-\mathrm{O}(23)$ & $102.0(9)$ & $\mathrm{O}(59)-\mathrm{W}(15)-\mathrm{O}(14)$ & $158.1(8)$ & $\mathrm{W}(15)-\mathrm{O}(14)-\mathrm{W}(14)$ & $122.2(11)$ \\
\hline $\mathrm{O}(42)-\mathrm{W}(6)-\mathrm{O}(25)$ & $101.5(8)$ & $\mathrm{O}(3)-\mathrm{W}(15)-\mathrm{O}(14)$ & $90.7(9)$ & $\mathrm{W}(1)-\mathrm{O}(15)-\mathrm{W}(10)$ & $144.5(10)$ \\
\hline $\mathrm{O}(23)-\mathrm{W}(6)-\mathrm{O}(25)$ & $90.9(8)$ & $\mathrm{O}(53)-\mathrm{W}(15)-\mathrm{O}(14)$ & $86.9(8)$ & $\mathrm{P}(2)-\mathrm{O}(16)-\mathrm{W}(9)$ & $129.2(11)$ \\
\hline $\mathrm{O}(42)-\mathrm{W}(6)-\mathrm{O}(55)$ & $98.6(7)$ & $\mathrm{O}(5)-\mathrm{W}(15)-\mathrm{O}(63)$ & 169.1(9) & $\mathrm{P}(2)-\mathrm{O}(16)-\mathrm{W}(7)$ & $127.8(10)$ \\
\hline $\mathrm{O}(23)-\mathrm{W}(6)-\mathrm{O}(55)$ & $94.8(7)$ & $\mathrm{O}(59)-\mathrm{W}(15)-\mathrm{O}(63)$ & $85.7(7)$ & $\mathrm{W}(9)-\mathrm{O}(16)-\mathrm{W}(7)$ & $90.0(6)$ \\
\hline $\mathrm{O}(25)-\mathrm{W}(6)-\mathrm{O}(55)$ & $157.4(7)$ & $\mathrm{O}(3)-\mathrm{W}(15)-\mathrm{O}(63)$ & $71.8(8)$ & $\mathrm{P}(1)-\mathrm{O}(18)-\mathrm{W}(2)$ & $127.8(10)$ \\
\hline $\mathrm{O}(42)-\mathrm{W}(6)-\mathrm{O}(19)$ & $94.7(9)$ & $\mathrm{O}(53)-\mathrm{W}(15)-\mathrm{O}(63)$ & $83.5(7)$ & $\mathrm{P}(1)-\mathrm{O}(18)-\mathrm{W}(10)$ & $126.2(10)$ \\
\hline $\mathrm{O}(23)-\mathrm{W}(6)-\mathrm{O}(19)$ & $163.2(8)$ & $\mathrm{O}(14)-\mathrm{W}(15)-\mathrm{O}(63)$ & $72.6(7)$ & $\mathrm{W}(2)-\mathrm{O}(18)-\mathrm{W}(10)$ & $91.7(6)$ \\
\hline $\mathrm{O}(25)-\mathrm{W}(6)-\mathrm{O}(19)$ & $84.4(7)$ & $\mathrm{O}(70)-\mathrm{Gd}(1)-\mathrm{O}(76)$ & $140.3(9)$ & $\mathrm{W}(14)-\mathrm{O}(19)-\mathrm{W}(6)$ & $148.1(10)$ \\
\hline $\mathrm{O}(55)-\mathrm{W}(6)-\mathrm{O}(19)$ & $83.9(7)$ & $\mathrm{O}(70)-\mathrm{Gd}(1)-\mathrm{O}(2)$ & $77.7(9)$ & $\mathrm{W}(14)-\mathrm{O}(20)-\mathrm{W}(9)$ & $148.8(11)$ \\
\hline $\mathrm{O}(42)-\mathrm{W}(6)-\mathrm{O}(49)$ & $170.2(8)$ & $\mathrm{O}(76)-\mathrm{Gd}(1)-\mathrm{O}(2)$ & $78.1(9)$ & $\mathrm{W}(6)-\mathrm{O}(23)-\mathrm{W}(8)$ & $167.2(11)$ \\
\hline $\mathrm{O}(23)-\mathrm{W}(6)-\mathrm{O}(49)$ & $84.2(7)$ & $\mathrm{O}(70)-\mathrm{Gd}(1)-\mathrm{O}(68)$ & $70.2(12)$ & $\mathrm{W}(9)-\mathrm{O}(24)-\mathrm{W}(2)$ & $165.5(11)$ \\
\hline $\mathrm{O}(25)-\mathrm{W}(6)-\mathrm{O}(49)$ & $85.9(7)$ & $\mathrm{O}(76)-\mathrm{Gd}(1)-\mathrm{O}(68)$ & $138.2(12)$ & $\mathrm{W}(6)-\mathrm{O}(25)-\mathrm{W}(9)$ & $155.2(10)$ \\
\hline $\mathrm{O}(55)-\mathrm{W}(6)-\mathrm{O}(49)$ & $73.1(6)$ & $\mathrm{O}(2)-\mathrm{Gd}(1)-\mathrm{O}(68)$ & $143.7(12)$ & $\mathrm{W}(13)-\mathrm{O}(27)-\mathrm{W}(14)$ & $124.5(11)$ \\
\hline $\mathrm{O}(19)-\mathrm{W}(6)-\mathrm{O}(49)$ & $79.4(7)$ & $\mathrm{O}(70)-\mathrm{Gd}(1)-\mathrm{O}(69)$ & $70.1(10)$ & $\mathrm{W}(13)-\mathrm{O}(28)-\mathrm{W}(4)$ & $147.8(12)$ \\
\hline $\mathrm{O}(10)-\mathrm{W}(7)-\mathrm{O}(57)$ & $101.3(10)$ & $\mathrm{O}(76)-\mathrm{Gd}(1)-\mathrm{O}(69)$ & $132.8(10)$ & $\mathrm{W}(12)-\mathrm{O}(31)-\mathrm{Co}(2)$ & $136.9(10)$ \\
\hline $\mathrm{O}(10)-\mathrm{W}(7)-\mathrm{O}(36)$ & $101.8(10)$ & $\mathrm{O}(2)-\mathrm{Gd}(1)-\mathrm{O}(69)$ & $77.1(10)$ & $\mathrm{W}(3)-\mathrm{O}(32)-\mathrm{W}(12)$ & $145.1(11)$ \\
\hline $\mathrm{O}(57)-\mathrm{W}(7)-\mathrm{O}(36)$ & $90.9(8)$ & $\mathrm{O}(68)-\mathrm{Gd}(1)-\mathrm{O}(69)$ & $76.4(13)$ & $\mathrm{W}(11)-\mathrm{O}(33)-\mathrm{W}(5)$ & $120.2(9)$ \\
\hline $\mathrm{O}(10)-\mathrm{W}(7)-\mathrm{O}(44)$ & $99.8(10)$ & $\mathrm{O}(70)-\mathrm{Gd}(1)-\mathrm{O}(60)$ & $128.6(8)$ & $\mathrm{W}(7)-\mathrm{O}(36)-\mathrm{W}(5)$ & $155.6(12)$ \\
\hline $\mathrm{O}(57)-\mathrm{W}(7)-\mathrm{O}(44)$ & $90.9(9)$ & $\mathrm{O}(76)-\mathrm{Gd}(1)-\mathrm{O}(60)$ & $68.9(7)$ & $\mathrm{W}(3)-\mathrm{O}(37)-\mathrm{Co}(1)$ & $135.6(10)$ \\
\hline $\mathrm{O}(36)-\mathrm{W}(7)-\mathrm{O}(44)$ & $157.5(8)$ & $\mathrm{O}(2)-\mathrm{Gd}(1)-\mathrm{O}(60)$ & $69.2(6)$ & $\mathrm{P}(1)-\mathrm{O}(38)-\mathrm{W}(3)$ & $128.1(10)$ \\
\hline $\mathrm{O}(10)-\mathrm{W}(7)-\mathrm{O}(53)$ & $97.3(10)$ & $\mathrm{O}(68)-\mathrm{Gd}(1)-\mathrm{O}(60)$ & $119.4(10)$ & $\mathrm{P}(1)-\mathrm{O}(38)-\mathrm{W}(8)$ & $126.4(10)$ \\
\hline $\mathrm{O}(57)-\mathrm{W}(7)-\mathrm{O}(53)$ & $161.3(8)$ & $\mathrm{O}(69)-\mathrm{Gd}(1)-\mathrm{O}(60)$ & $65.0(9)$ & $\mathrm{W}(3)-\mathrm{O}(38)-\mathrm{W}(8)$ & $90.1(6)$ \\
\hline $\mathrm{O}(36)-\mathrm{W}(7)-\mathrm{O}(53)$ & $83.7(8)$ & $\mathrm{O}(70)-\mathrm{Gd}(1)-\mathrm{O}(6)$ & $70.1(9)$ & $\mathrm{W}(1)-\mathrm{O}(39)-\mathrm{Gd}(2)$ & $155.6(10)$ \\
\hline $\mathrm{O}(44)-\mathrm{W}(7)-\mathrm{O}(53)$ & $87.4(8)$ & $\mathrm{O}(76)-\mathrm{Gd}(1)-\mathrm{O}(6)$ & $72.7(8)$ & $\mathrm{W}(6)-\mathrm{O}(42)-\mathrm{Co}(3)$ & $151.7(11)$ \\
\hline $\mathrm{O}(10)-\mathrm{W}(7)-\mathrm{O}(16)$ & $172.7(9)$ & $\mathrm{O}(2)-\mathrm{Gd}(1)-\mathrm{O}(6)$ & $71.7(7)$ & $\mathrm{W}(9)-\mathrm{O}(44)-\mathrm{W}(7)$ & $121.6(10)$ \\
\hline $\mathrm{O}(57)-\mathrm{W}(7)-\mathrm{O}(16)$ & $81.2(7)$ & $\mathrm{O}(68)-\mathrm{Gd}(1)-\mathrm{O}(6)$ & $111.2(11)$ & $\mathrm{P}(2)-\mathrm{O}(45)-\mathrm{W}(5)$ & $130.2(10)$ \\
\hline $\mathrm{O}(36)-\mathrm{W}(7)-\mathrm{O}(16)$ & $84.9(7)$ & $\mathrm{O}(69)-\mathrm{Gd}(1)-\mathrm{O}(6)$ & $133.4(9)$ & $\mathrm{P}(2)-\mathrm{O}(45)-\mathrm{W}(11)$ & $128.4(10)$ \\
\hline $\mathrm{O}(44)-\mathrm{W}(7)-\mathrm{O}(16)$ & $73.2(7)$ & $\mathrm{O}(60)-\mathrm{Gd}(1)-\mathrm{O}(6)$ & $129.4(7)$ & $\mathrm{W}(5)-\mathrm{O}(45)-\mathrm{W}(11)$ & $90.9(6)$ \\
\hline $\mathrm{O}(53)-\mathrm{W}(7)-\mathrm{O}(16)$ & $80.5(7)$ & $\mathrm{O}(70)-\mathrm{Gd}(1)-\mathrm{O}(71)$ & $140.0(10)$ & $\mathrm{W}(1)-\mathrm{O}(46)-\mathrm{Co}(1)^{\mathrm{i}}$ & $128.8(9)$ \\
\hline $\mathrm{O}(43)-\mathrm{W}(8)-\mathrm{O}(58)$ & $100.6(9)$ & $\mathrm{O}(76)-\mathrm{Gd}(1)-\mathrm{O}(71)$ & $77.8(9)$ & $\mathrm{W}(1)-\mathrm{O}(46)-\mathrm{Co}(2)$ & $130.9(9)$ \\
\hline $\mathrm{O}(43)-\mathrm{W}(8)-\mathrm{O}(50)$ & $100.0(8)$ & $\mathrm{O}(2)-\mathrm{Gd}(1)-\mathrm{O}(71)$ & $134.5(8)$ & $\mathrm{Co}(1) \mathrm{i}-\mathrm{O}(46)-\mathrm{Co}(2)$ & $97.7(8)$ \\
\hline
\end{tabular}




\begin{tabular}{|c|c|c|c|c|c|}
\hline $\mathrm{O}(58)-\mathrm{W}(8)-\mathrm{O}(50)$ & $94.5(8)$ & $\mathrm{O}(68)-\mathrm{Gd}(1)-\mathrm{O}(71)$ & $70.8(12)$ & $\mathrm{W}(4)-\mathrm{O}(47)-\mathrm{W}(3)$ & $163.3(11)$ \\
\hline $\mathrm{O}(43)-\mathrm{W}(8)-\mathrm{O}(54)$ & 101.7(8) & $\mathrm{O}(69)-\mathrm{Gd}(1)-\mathrm{O}(71)$ & $92.2(10)$ & $\mathrm{W}(10)-\mathrm{O}(48)-\mathrm{Co}(1)^{\mathrm{i}}$ & $128.8(10)$ \\
\hline $\mathrm{O}(58)-\mathrm{W}(8)-\mathrm{O}(54)$ & $95.9(8)$ & $\mathrm{O}(60)-\mathrm{Gd}(1)-\mathrm{O}(71)$ & $66.3(7)$ & $\mathrm{W}(10)-\mathrm{O}(48)-\mathrm{Co}(2)^{\mathrm{i}}$ & $131.7(10)$ \\
\hline $\mathrm{O}(50)-\mathrm{W}(8)-\mathrm{O}(54)$ & $153.7(8)$ & $\mathrm{O}(6)-\mathrm{Gd}(1)-\mathrm{O}(71)$ & 134.3(8) & $\mathrm{Co}(1)^{\mathrm{i}}-\mathrm{O}(48)-\mathrm{Co}(2)^{\mathrm{i}}$ & $97.4(8)$ \\
\hline $\mathrm{O}(43)-\mathrm{W}(8)-\mathrm{O}(23)$ & $94.3(9)$ & $\mathrm{O}(70)-\mathrm{Gd}(1)-\mathrm{O}(67)$ & $104.2(11)$ & $\mathrm{P}(2)-\mathrm{O}(49)-\mathrm{W}(4)$ & $128.0(9)$ \\
\hline $\mathrm{O}(58)-\mathrm{W}(8)-\mathrm{O}(23)$ & $165.1(8)$ & $\mathrm{O}(76)-\mathrm{Gd}(1)-\mathrm{O}(67)$ & $74.1(11)$ & $\mathrm{P}(2)-\mathrm{O}(49)-\mathrm{W}(6)$ & $129.8(9)$ \\
\hline $\mathrm{O}(50)-\mathrm{W}(8)-\mathrm{O}(23)$ & $82.5(7)$ & $\mathrm{O}(2)-\mathrm{Gd}(1)-\mathrm{O}(67)$ & $136.8(10)$ & $\mathrm{W}(4)-\mathrm{O}(49)-\mathrm{W}(6)$ & $91.9(6)$ \\
\hline $\mathrm{O}(54)-\mathrm{W}(8)-\mathrm{O}(23)$ & $81.3(8)$ & $\mathrm{O}(68)-\mathrm{Gd}(1)-\mathrm{O}(67)$ & $69.6(14)$ & $\mathrm{W}(8)-\mathrm{O}(50)-\mathrm{W}(3)$ & 119.2(9) \\
\hline $\mathrm{O}(43)-\mathrm{W}(8)-\mathrm{O}(38)$ & $173.9(8)$ & $\mathrm{O}(69)-\mathrm{Gd}(1)-\mathrm{O}(67)$ & $145.1(12)$ & $\mathrm{W}(5)-\mathrm{O}(51)-\mathrm{W}(1)$ & $164.1(11)$ \\
\hline $\mathrm{O}(58)-\mathrm{W}(8)-\mathrm{O}(38)$ & $82.7(8)$ & $\mathrm{O}(60)-\mathrm{Gd}(1)-\mathrm{O}(67)$ & $127.0(9)$ & $\mathrm{W}(12)-\mathrm{O}(52)-\mathrm{W}(1)$ & $121.8(9)$ \\
\hline $\mathrm{O}(50)-\mathrm{W}(8)-\mathrm{O}(38)$ & $74.5(7)$ & $\mathrm{O}(6)-\mathrm{Gd}(1)-\mathrm{O}(67)$ & $68.8(10)$ & $\mathrm{W}(15)-\mathrm{O}(53)-\mathrm{W}(7)$ & $149.9(11)$ \\
\hline $\mathrm{O}(54)-\mathrm{W}(8)-\mathrm{O}(38)$ & $83.0(7)$ & $\mathrm{O}(71)-\mathrm{Gd}(1)-\mathrm{O}(67)$ & $69.9(10)$ & $\mathrm{W}(2)-\mathrm{O}(54)-\mathrm{W}(8)$ & $146.4(11)$ \\
\hline $\mathrm{O}(23)-\mathrm{W}(8)-\mathrm{O}(38)$ & $82.4(7)$ & $\mathrm{O}(35)-\mathrm{Gd}(2)-\mathrm{O}(7)$ & $140.3(7)$ & $\mathrm{W}(4)-\mathrm{O}(55)-\mathrm{W}(6)$ & $119.0(8)$ \\
\hline $\mathrm{O}(41)-\mathrm{W}(9)-\mathrm{O}(24)$ & $102.2(10)$ & $\mathrm{O}(35)-\mathrm{Gd}(2)-\mathrm{O}(39)$ & $142.3(7)$ & $\mathrm{W}(13)-\mathrm{O}(56)-\mathrm{W}(11)$ & $149.1(12)$ \\
\hline $\mathrm{O}(41)-\mathrm{W}(9)-\mathrm{O}(44)$ & $97.6(9)$ & $\mathrm{O}(7)-\mathrm{Gd}(2)-\mathrm{O}(39)$ & $69.9(7)$ & $\mathrm{W}(7)-\mathrm{O}(57)-\mathrm{W}(10)$ & $164.8(11)$ \\
\hline $\mathrm{O}(24)-\mathrm{W}(9)-\mathrm{O}(44)$ & $94.4(9)$ & $\mathrm{O}(35)-\mathrm{Gd}(2)-\mathrm{O}(13)$ & $70.4(7)$ & $\mathrm{W}(8)-\mathrm{O}(58)-\mathrm{Co}(1)$ & $137.2(10)$ \\
\hline $\mathrm{O}(41)-\mathrm{W}(9)-\mathrm{O}(25)$ & 101.7(9) & $\mathrm{O}(7)-\mathrm{Gd}(2)-\mathrm{O}(13)$ & $98.5(7)$ & $\mathrm{W}(15)-\mathrm{O}(59)-\mathrm{W}(5)$ & $149.2(10)$ \\
\hline $\mathrm{O}(24)-\mathrm{W}(9)-\mathrm{O}(25)$ & $90.0(8)$ & $\mathrm{O}(39)-\mathrm{Gd}(2)-\mathrm{O}(13)$ & $138.8(6)$ & $\mathrm{W}(4)-\mathrm{O}(60)-\mathrm{Gd}(1)$ & $174.4(11)$ \\
\hline $\mathrm{O}(44)-\mathrm{W}(9)-\mathrm{O}(25)$ & $158.7(8)$ & $\mathrm{O}(35)-\mathrm{Gd}(2)-\mathrm{O}(66)$ & $79.3(8)$ & $\mathrm{W}(10)-\mathrm{O}(61)-\mathrm{W}(2)$ & $121.8(9)$ \\
\hline $\mathrm{O}(41)-\mathrm{W}(9)-\mathrm{O}(20)$ & $95.6(9)$ & $\mathrm{O}(7)-\mathrm{Gd}(2)-\mathrm{O}(66)$ & $137.2(8)$ & $\mathrm{W}(11)-\mathrm{O}(62)-\mathrm{W}(12)$ & $163.8(11)$ \\
\hline $\mathrm{O}(24)-\mathrm{W}(9)-\mathrm{O}(20)$ & $162.0(8)$ & $\mathrm{O}(39)-\mathrm{Gd}(2)-\mathrm{O}(66)$ & $84.8(7)$ & $\mathrm{P}(2)-\mathrm{O}(63)-\mathrm{W}(14)$ & $124.0(9)$ \\
\hline $\mathrm{O}(44)-\mathrm{W}(9)-\mathrm{O}(20)$ & $86.2(8)$ & $\mathrm{O}(13)-\mathrm{Gd}(2)-\mathrm{O}(66)$ & $78.0(7)$ & $\mathrm{P}(2)-\mathrm{O}(63)-\mathrm{W}(15)$ & $123.7(9)$ \\
\hline $\mathrm{O}(25)-\mathrm{W}(9)-\mathrm{O}(20)$ & $83.4(8)$ & $\mathrm{O}(35)-\mathrm{Gd}(2)-\mathrm{O}(78)$ & $131.7(8)$ & $\mathrm{W}(14)-\mathrm{O}(63)-\mathrm{W}(15)$ & $92.9(6)$ \\
\hline $\mathrm{O}(41)-\mathrm{W}(9)-\mathrm{O}(16)$ & 171.2(9) & $\mathrm{O}(7)-\mathrm{Gd}(2)-\mathrm{O}(78)$ & $69.7(8)$ & $\mathrm{P}(2)-\mathrm{O}(63)-\mathrm{W}(13)$ & 123.1(9) \\
\hline $\mathrm{O}(24)-\mathrm{W}(9)-\mathrm{O}(16)$ & $82.8(8)$ & $\mathrm{O}(39)-\mathrm{Gd}(2)-\mathrm{O}(78)$ & $71.3(8)$ & $\mathrm{W}(14)-\mathrm{O}(63)-\mathrm{W}(13)$ & $92.4(6)$ \\
\hline $\mathrm{O}(44)-\mathrm{W}(9)-\mathrm{O}(16)$ & $74.6(7)$ & $\mathrm{O}(13)-\mathrm{Gd}(2)-\mathrm{O}(78)$ & $67.7(8)$ & $\mathrm{W}(15)-\mathrm{O}(63)-\mathrm{W}(13)$ & $91.8(6)$ \\
\hline $\mathrm{O}(25)-\mathrm{W}(9)-\mathrm{O}(16)$ & $85.4(7)$ & $\mathrm{O}(66)-\mathrm{Gd}(2)-\mathrm{O}(78)$ & $69.7(9)$ & $\mathrm{W}(2)-\mathrm{O}(64)-\mathrm{Co}(2)^{\mathrm{i}}$ & $136.9(10)$ \\
\hline $\mathrm{O}(20)-\mathrm{W}(9)-\mathrm{O}(16)$ & $80.1(7)$ & $\mathrm{O}(35)-\mathrm{Gd}(2)-\mathrm{O}(26)$ & $87.9(6)$ & $\mathrm{P}(1)-\mathrm{O}(65)-\mathrm{Co}(2)^{\mathrm{i}}$ & $123.1(10)$ \\
\hline $\mathrm{O}(8)-\mathrm{W}(10)-\mathrm{O}(48)$ & 101.1(9) & $\mathrm{O}(7)-\mathrm{Gd}(2)-\mathrm{O}(26)$ & $76.4(7)$ & $\mathrm{P}(1)-\mathrm{O}(65)-\mathrm{Co}(2)$ & $124.1(11)$ \\
\hline $\mathrm{O}(8)-\mathrm{W}(10)-\mathrm{O}(61)$ & $101.9(8)$ & $\mathrm{O}(39)-\mathrm{Gd}(2)-\mathrm{O}(26)$ & $78.1(6)$ & $\mathrm{Co}(2)^{\mathrm{i}}-\mathrm{O}(65)-\mathrm{Co}(2)$ & $95.5(8)$ \\
\hline $\mathrm{O}(48)-\mathrm{W}(10)-\mathrm{O}(61)$ & $95.1(8)$ & $\mathrm{O}(13)-\mathrm{Gd}(2)-\mathrm{O}(26)$ & $139.4(6)$ & $\mathrm{P}(1)-\mathrm{O}(65)-\mathrm{Co}(1)$ & $120.5(11)$ \\
\hline $\mathrm{O}(8)-\mathrm{W}(10)-\mathrm{O}(15)$ & $100.9(8)$ & $\mathrm{O}(66)-\mathrm{Gd}(2)-\mathrm{O}(26)$ & $132.5(7)$ & $\mathrm{Co}(2)^{\mathrm{i}}-\mathrm{O}(65)-\mathrm{Co}(1)$ & $94.2(8)$ \\
\hline $\mathrm{O}(48)-\mathrm{W}(10)-\mathrm{O}(15)$ & $91.7(8)$ & $\mathrm{O}(78)-\mathrm{Gd}(2)-\mathrm{O}(26)$ & $140.2(8)$ & $\mathrm{Co}(2)-\mathrm{O}(65)-\mathrm{Co}(1)$ & $91.4(7)$ \\
\hline $\mathrm{O}(61)-\mathrm{W}(10)-\mathrm{O}(15)$ & $154.5(7)$ & $\mathrm{O}(35)-\mathrm{Gd}(2)-\mathrm{O}(34)$ & $70.0(7)$ & & \\
\hline $\mathrm{O}(8)-\mathrm{W}(10)-\mathrm{O}(57)$ & $93.7(9)$ & $\mathrm{O}(7)-\mathrm{Gd}(2)-\mathrm{O}(34)$ & $70.5(7)$ & & \\
\hline
\end{tabular}

Symmetry transformations used to generate equivalent atoms: i) $-x+1 / 2,-y+1 / 2,-z$. 
Table S4. Selected Bond lengths $[\AA]$ for $\left\{\mathrm{Gd}_{4} \mathrm{Mn}_{6} \mathrm{P}_{4} \mathrm{~W}_{30}\right\}$.

\begin{tabular}{|c|c|c|c|c|c|}
\hline Bond lengths & (Å) & Bond lengths & $(\AA ̊)$ & Bond lengths & (Å) \\
\hline $\mathrm{W}(1)-\mathrm{O}(26)$ & $1.723(16)$ & $\mathrm{W}(8)-\mathrm{O}(30)$ & $1.913(13)$ & $\mathrm{P}(1)-\mathrm{O}(37)$ & $1.512(15)$ \\
\hline $\mathrm{W}(1)-\mathrm{O}(54)$ & $1.784(16)$ & $\mathrm{W}(8)-\mathrm{O}(34)$ & $1.926(18)$ & $\mathrm{P}(1)-\mathrm{O}(36)$ & $1.547(16)$ \\
\hline $\mathrm{W}(1)-\mathrm{O}(27)$ & $1.881(13)$ & $\mathrm{W}(8)-\mathrm{O}(36)$ & $2.354(13)$ & $\mathrm{P}(1)-\mathrm{O}(7)$ & $1.565(13)$ \\
\hline $\mathrm{W}(1)-\mathrm{O}(24)$ & $1.950(12)$ & $\mathrm{W}(9)-\mathrm{O}(29)$ & $1.699(14)$ & $\mathrm{P}(1)-\mathrm{O}(40)$ & $1.582(18)$ \\
\hline $\mathrm{W}(1)-\mathrm{O}(14)$ & $2.009(18)$ & $\mathrm{W}(9)-\mathrm{O}(42)$ & $1.770(17)$ & $\mathrm{P}(2)-\mathrm{O}(58)$ & $1.534(17)$ \\
\hline $\mathrm{W}(1)-\mathrm{O}(37)$ & $2.373(15)$ & $\mathrm{W}(9)-\mathrm{O}(45)$ & $1.930(12)$ & $\mathrm{P}(2)-\mathrm{O}(20)$ & $1.534(14)$ \\
\hline $\mathrm{W}(2)-\mathrm{O}(25)$ & $1.711(15)$ & $\mathrm{W}(9)-\mathrm{O}(15)$ & $1.937(13)$ & $\mathrm{P}(2)-\mathrm{O}(19)$ & $1.536(13)$ \\
\hline $\mathrm{W}(2)-\mathrm{O}(48)$ & $1.841(17)$ & $\mathrm{W}(9)-\mathrm{O}(54)$ & $2.094(16)$ & $\mathrm{P}(2)-\mathrm{O}(8)$ & $1.543(14)$ \\
\hline $\mathrm{W}(2)-\mathrm{O}(18)$ & $1.878(14)$ & $\mathrm{W}(9)-\mathrm{O}(20)$ & $2.375(12)$ & $\mathrm{Gd}(1)-\mathrm{O}(70)$ & $2.40(2)$ \\
\hline $\mathrm{W}(2)-\mathrm{O}(41)$ & $1.896(14)$ & $\mathrm{W}(10)-\mathrm{O}(33)$ & $1.716(14)$ & $\mathrm{Gd}(1)-\mathrm{O}(62)$ & $2.43(4)$ \\
\hline $\mathrm{W}(2)-\mathrm{O}(50)$ & $1.958(17)$ & $\mathrm{W}(10)-\mathrm{O}(56)$ & $1.794(16)$ & $\mathrm{Gd}(1)-\mathrm{O}(64)$ & $2.44(3)$ \\
\hline $\mathrm{W}(2)-\mathrm{O}(7)$ & $2.353(13)$ & $\mathrm{W}(10)-\mathrm{O}(23)$ & $1.896(14)$ & $\mathrm{Gd}(1)-\mathrm{O}(67)$ & $2.45(2)$ \\
\hline $\mathrm{W}(3)-\mathrm{O}(31)$ & $1.701(14)$ & $\mathrm{W}(10)-\mathrm{O}(24)$ & $1.911(13)$ & $\mathrm{Gd}(1)-\mathrm{O}(71)$ & $2.46(2)$ \\
\hline $\mathrm{W}(3)-\mathrm{O}(43)$ & $1.757(17)$ & $\mathrm{W}(10)-\mathrm{O}(28)$ & $1.988(18)$ & $\mathrm{Gd}(1)-\mathrm{O}(72)$ & $2.461(19)$ \\
\hline $\mathrm{W}(3)-\mathrm{O}(15)$ & $1.866(13)$ & $\mathrm{W}(10)-\mathrm{O}(37)$ & $2.363(14)$ & $\mathrm{Gd}(1)-\mathrm{O}(63)$ & $2.46(2)$ \\
\hline $\mathrm{W}(3)-\mathrm{O}(46)$ & $1.938(12)$ & $\mathrm{W}(11)-\mathrm{O}(9)$ & $1.714(14)$ & $\mathrm{Gd}(1)-\mathrm{O}(33)$ & $2.478(14)$ \\
\hline $\mathrm{W}(3)-\mathrm{O}(55)$ & $2.051(16)$ & $\mathrm{W}(11)-\mathrm{O}(52)$ & $1.807(15)$ & $\mathrm{Gd}(1)-\mathrm{O}(68)$ & $2.484(18)$ \\
\hline W(3)-O(19) & $2.345(12)$ & $\mathrm{W}(11)-\mathrm{O}(46)$ & $1.914(12)$ & $\mathrm{Gd}(2)-\mathrm{O}(3)$ & $2.392(18)$ \\
\hline $\mathrm{W}(4)-\mathrm{O}(17)$ & $1.716(13)$ & $\mathrm{W}(11)-\mathrm{O}(35)$ & $1.930(12)$ & $\mathrm{Gd}(2)-\mathrm{O}(39)$ & $2.413(14)$ \\
\hline $\mathrm{W}(4)-\mathrm{O}(47)$ & $1.790(17)$ & $\mathrm{W}(11)-\mathrm{O}(48)$ & $1.987(17)$ & $\mathrm{Gd}(2)-\mathrm{O}(76)$ & $2.43(2)$ \\
\hline $\mathrm{W}(4)-\mathrm{O}(12)$ & $1.885(13)$ & $\mathrm{W}(11)-\mathrm{O}(19)$ & $2.370(13)$ & $\mathrm{Gd}(2)-\mathrm{O}(74)$ & $2.441(18)$ \\
\hline $\mathrm{W}(4)-\mathrm{O}(45)$ & $1.929(12)$ & $\mathrm{W}(12)-\mathrm{O}(75)$ & $1.704(13)$ & $\mathrm{Gd}(2)-\mathrm{O}(69)$ & $2.450(19)$ \\
\hline $\mathrm{W}(4)-\mathrm{O}(56)$ & $2.050(16)$ & $\mathrm{W}(12)-\mathrm{O}(51)$ & $1.778(16)$ & $\mathrm{Gd}(2)-\mathrm{O}(73)$ & $2.459(16)$ \\
\hline $\mathrm{W}(4)-\mathrm{O}(20)$ & $2.345(12)$ & $\mathrm{W}(12)-\mathrm{O}(12)$ & $1.905(14)$ & $\mathrm{Gd}(2)-\mathrm{O}(77)$ & $2.477(15)$ \\
\hline $\mathrm{W}(5)-\mathrm{O}(2)$ & $1.719(16)$ & $\mathrm{W}(12)-\mathrm{O}(21)$ & $1.936(12)$ & $\mathrm{Gd}(2)-\mathrm{O}(11)$ & $2.482(13)$ \\
\hline $\mathrm{W}(5)-\mathrm{O}(55)$ & $1.806(16)$ & $\mathrm{W}(12)-\mathrm{O}(53)$ & $2.040(17)$ & $\mathrm{Gd}(2)-\mathrm{O}(78)$ & $2.495(16)$ \\
\hline $\mathrm{W}(5)-\mathrm{O}(27)$ & $1.899(14)$ & $\mathrm{W}(12)-\mathrm{O}(8)$ & $2.360(13)$ & $\mathrm{Mn}(1)-\mathrm{O}(42)$ & $2.107(15)$ \\
\hline $\mathrm{W}(5)-\mathrm{O}(41)$ & $1.903(14)$ & $\mathrm{W}(13)-\mathrm{O}(6)$ & $1.718(17)$ & $\mathrm{Mn}(1)-\mathrm{O}(47)$ & $2.121(15)$ \\
\hline $\mathrm{W}(5)-\mathrm{O}(38)$ & $1.993(17)$ & $\mathrm{W}(13)-\mathrm{O}(28)$ & $1.865(16)$ & $\operatorname{Mn}(1)-O(52)^{i}$ & $2.149(15)$ \\
\hline $\mathrm{W}(5)-\mathrm{O}(7)$ & $2.339(13)$ & $\mathrm{W}(13)-\mathrm{O}(34)$ & $1.930(17)$ & $\operatorname{Mn}(1)-O(32)^{i}$ & $2.201(14)$ \\
\hline $\mathrm{W}(6)-\mathrm{O}(39)$ & $1.723(14)$ & $\mathrm{W}(13)-\mathrm{O}(1)$ & $1.962(13)$ & $\mathrm{Mn}(1)-\mathrm{O}(49)$ & $2.206(14)$ \\
\hline $\mathrm{W}(6)-\mathrm{O}(32)$ & $1.781(15)$ & $\mathrm{W}(13)-\mathrm{O}(5)$ & $2.038(12)$ & $\mathrm{Mn}(1)-\mathrm{O}(58)$ & $2.275(14)$ \\
\hline $\mathrm{W}(6)-\mathrm{O}(35)$ & $1.887(13)$ & $\mathrm{W}(13)-\mathrm{O}(40)$ & $2.390(14)$ & $\mathrm{Mn}(2)-\mathrm{O}(51)$ & $2.089(14)$ \\
\hline $\mathrm{W}(6)-\mathrm{O}(21)$ & $1.908(12)$ & $\mathrm{W}(14)-\mathrm{O}(10)$ & $1.71(2)$ & $\mathrm{Mn}(2)-\mathrm{O}(43)^{\mathrm{i}}$ & $2.095(16)$ \\
\hline $\mathrm{W}(6)-\mathrm{O}(57)$ & $2.069(16)$ & $\mathrm{W}(14)-\mathrm{O}(5)$ & $1.775(12)$ & $\operatorname{Mn}(2)-O(52)^{\mathrm{i}}$ & $2.170(13)$ \\
\hline $\mathrm{W}(6)-\mathrm{O}(8)$ & $2.325(13)$ & $\mathrm{W}(14)-\mathrm{O}(44)$ & $1.884(16)$ & $\mathrm{Mn}(2)-\mathrm{O}(32)$ & $2.181(14)$ \\
\hline $\mathrm{W}(7)-\mathrm{O}(22)$ & $1.720(16)$ & $\mathrm{W}(14)-\mathrm{O}(50)$ & $1.902(15)$ & $\operatorname{Mn}(2)-O(58)^{i}$ & $2.262(14)$ \\
\hline $\mathrm{W}(7)-\mathrm{O}(57)$ & $1.780(16)$ & $\mathrm{W}(14)-\mathrm{O}(4)$ & $1.91(2)$ & $\mathrm{Mn}(2)-\mathrm{O}(58)$ & $2.264(16)$ \\
\hline $\mathrm{W}(7)-\mathrm{O}(18)$ & $1.908(14)$ & $\mathrm{W}(14)-\mathrm{O}(40)$ & $2.354(15)$ & $\mathrm{Mn}(3)-\mathrm{O}(65)$ & $2.04(3)$ \\
\hline $\mathrm{W}(7)-\mathrm{O}(30)$ & $1.934(13)$ & $\mathrm{W}(15)-\mathrm{O}(13)$ & $1.73(2)$ & $\mathrm{Mn}(3)-\mathrm{O}(61)$ & $2.05(3)$ \\
\hline $\mathrm{W}(7)-\mathrm{O}(44)$ & $1.960(17)$ & $\mathrm{W}(15)-\mathrm{O}(1)$ & $1.761(12)$ & $\mathrm{Mn}(3)-\mathrm{O}(60)$ & $2.15(3)$ \\
\hline $\mathrm{W}(7)-\mathrm{O}(36)$ & $2.348(14)$ & $\mathrm{W}(15)-\mathrm{O}(14)$ & $1.857(16)$ & $\mathrm{Mn}(3)-\mathrm{O}(59)$ & $2.16(4)$ \\
\hline $\mathrm{W}(8)-\mathrm{O}(16)$ & $1.723(14)$ & $\mathrm{W}(15)-\mathrm{O}(38)$ & $1.897(16)$ & $\mathrm{Mn}(3)-\mathrm{O}(26)$ & $2.244(16)$ \\
\hline $\mathrm{W}(8)-\mathrm{O}(53)$ & $1.816(18)$ & $\mathrm{W}(15)-\mathrm{O}(4)$ & $1.927(19)$ & $\mathrm{Mn}(3)-\mathrm{O}(66)$ & $2.32(2)$ \\
\hline $\mathrm{W}(8)-\mathrm{O}(23)$ & $1.879(13)$ & $\mathrm{W}(15)-\mathrm{O}(40)$ & $2.362(16)$ & & \\
\hline
\end{tabular}

Symmetry transformations used to generate equivalent atoms: $i)-x-1 / 2,-y+3 / 2,-z+1$. 
Table S5. Selected Bond Angles for $\left\{\mathrm{Gd}_{4} \mathrm{Mn}_{6} \mathrm{P}_{4} \mathrm{~W}_{30}\right\}$.

\begin{tabular}{|c|c|c|c|c|c|}
\hline Bond angles & $\left({ }^{0}\right)$ & Bond angles & $\left({ }^{0}\right)$ & Bond angles & $\left({ }^{0}\right)$ \\
\hline $\mathrm{O}(26)-\mathrm{W}(1)-\mathrm{O}(54)$ & $101.9(7)$ & $\mathrm{O}(56)-\mathrm{W}(10)-\mathrm{O}(28)$ & $162.5(6)$ & $\mathrm{O}(3)-\mathrm{Gd}(2)-\mathrm{O}(73)$ & $69.8(6)$ \\
\hline $\mathrm{O}(26)-\mathrm{W}(1)-\mathrm{O}(27)$ & $102.1(6)$ & $\mathrm{O}(23)-\mathrm{W}(10)-\mathrm{O}(28)$ & $84.7(7)$ & $\mathrm{O}(39)-\mathrm{Gd}(2)-\mathrm{O}(73)$ & $139.7(5)$ \\
\hline $\mathrm{O}(54)-\mathrm{W}(1)-\mathrm{O}(27)$ & $92.8(7)$ & $\mathrm{O}(24)-\mathrm{W}(10)-\mathrm{O}(28)$ & $85.8(6)$ & $\mathrm{O}(76)-\mathrm{Gd}(2)-\mathrm{O}(73)$ & $99.9(6)$ \\
\hline $\mathrm{O}(26)-\mathrm{W}(1)-\mathrm{O}(24)$ & $97.9(6)$ & $\mathrm{O}(33)-\mathrm{W}(10)-\mathrm{O}(37)$ & $174.0(7)$ & $\mathrm{O}(74)-\mathrm{Gd}(2)-\mathrm{O}(73)$ & $77.0(6)$ \\
\hline $\mathrm{O}(54)-\mathrm{W}(1)-\mathrm{O}(24)$ & $93.8(6)$ & $\mathrm{O}(56)-\mathrm{W}(10)-\mathrm{O}(37)$ & $80.8(6)$ & $\mathrm{O}(69)-\mathrm{Gd}(2)-\mathrm{O}(73)$ & $67.8(6)$ \\
\hline $\mathrm{O}(27)-\mathrm{W}(1)-\mathrm{O}(24)$ & $157.2(6)$ & $\mathrm{O}(23)-\mathrm{W}(10)-\mathrm{O}(37)$ & $84.9(5)$ & $\mathrm{O}(3)-\mathrm{Gd}(2)-\mathrm{O}(77)$ & $69.5(6)$ \\
\hline $\mathrm{O}(26)-\mathrm{W}(1)-\mathrm{O}(14)$ & $94.3(8)$ & $\mathrm{O}(24)-\mathrm{W}(10)-\mathrm{O}(37)$ & $74.9(5)$ & $\mathrm{O}(39)-\mathrm{Gd}(2)-\mathrm{O}(77)$ & $133.0(5)$ \\
\hline $\mathrm{O}(54)-\mathrm{W}(1)-\mathrm{O}(14)$ & $163.8(6)$ & $\mathrm{O}(28)-\mathrm{W}(10)-\mathrm{O}(37)$ & $81.9(6)$ & $\mathrm{O}(76)-\mathrm{Gd}(2)-\mathrm{O}(77)$ & $70.4(6)$ \\
\hline $\mathrm{O}(27)-\mathrm{W}(1)-\mathrm{O}(14)$ & $82.7(6)$ & $\mathrm{O}(9)-\mathrm{W}(11)-\mathrm{O}(52)$ & $99.9(7)$ & $\mathrm{O}(74)-\mathrm{Gd}(2)-\mathrm{O}(77)$ & $141.3(6)$ \\
\hline $\mathrm{O}(24)-\mathrm{W}(1)-\mathrm{O}(14)$ & $84.9(6)$ & $\mathrm{O}(9)-\mathrm{W}(11)-\mathrm{O}(46)$ & 101.1(6) & $\mathrm{O}(69)-\mathrm{Gd}(2)-\mathrm{O}(77)$ & $113.0(6)$ \\
\hline $\mathrm{O}(26)-\mathrm{W}(1)-\mathrm{O}(37)$ & $170.7(7)$ & $\mathrm{O}(52)-\mathrm{W}(11)-\mathrm{O}(46)$ & $94.1(6)$ & $\mathrm{O}(73)-\mathrm{Gd}(2)-\mathrm{O}(77)$ & $69.9(5)$ \\
\hline $\mathrm{O}(54)-\mathrm{W}(1)-\mathrm{O}(37)$ & $83.5(6)$ & $\mathrm{O}(9)-\mathrm{W}(11)-\mathrm{O}(35)$ & $101.3(6)$ & $\mathrm{O}(3)-\mathrm{Gd}(2)-\mathrm{O}(11)$ & $88.5(5)$ \\
\hline $\mathrm{O}(27)-\mathrm{W}(1)-\mathrm{O}(37)$ & $85.0(6)$ & $\mathrm{O}(52)-\mathrm{W}(11)-\mathrm{O}(35)$ & $90.8(6)$ & $\mathrm{O}(39)-\mathrm{Gd}(2)-\mathrm{O}(11)$ & $76.7(5)$ \\
\hline $\mathrm{O}(24)-\mathrm{W}(1)-\mathrm{O}(37)$ & $74.0(5)$ & $\mathrm{O}(46)-\mathrm{W}(11)-\mathrm{O}(35)$ & $155.8(6)$ & $\mathrm{O}(76)-\mathrm{Gd}(2)-\mathrm{O}(11)$ & $74.8(6)$ \\
\hline $\mathrm{O}(14)-\mathrm{W}(1)-\mathrm{O}(37)$ & $80.6(6)$ & $\mathrm{O}(9)-\mathrm{W}(11)-\mathrm{O}(48)$ & $94.4(7)$ & $\mathrm{O}(74)-\mathrm{Gd}(2)-\mathrm{O}(11)$ & $134.2(5)$ \\
\hline $\mathrm{O}(25)-\mathrm{W}(2)-\mathrm{O}(48)$ & 101.2(8) & $\mathrm{O}(52)-\mathrm{W}(11)-\mathrm{O}(48)$ & $165.2(6)$ & $\mathrm{O}(69)-\mathrm{Gd}(2)-\mathrm{O}(11)$ & $138.4(6)$ \\
\hline $\mathrm{O}(25)-\mathrm{W}(2)-\mathrm{O}(18)$ & $101.0(7)$ & $\mathrm{O}(46)-\mathrm{W}(11)-\mathrm{O}(48)$ & $86.9(6)$ & $\mathrm{O}(73)-\mathrm{Gd}(2)-\mathrm{O}(11)$ & $139.6(5)$ \\
\hline $\mathrm{O}(48)-\mathrm{W}(2)-\mathrm{O}(18)$ & $89.4(7)$ & $\mathrm{O}(35)-\mathrm{W}(11)-\mathrm{O}(48)$ & $82.5(6)$ & $\mathrm{O}(77)-\mathrm{Gd}(2)-\mathrm{O}(11)$ & $70.8(5)$ \\
\hline $\mathrm{O}(25)-\mathrm{W}(2)-\mathrm{O}(41)$ & $99.8(7)$ & $\mathrm{O}(9)-\mathrm{W}(11)-\mathrm{O}(19)$ & $174.0(6)$ & $\mathrm{O}(3)-\mathrm{Gd}(2)-\mathrm{O}(78)$ & $71.3(5)$ \\
\hline $\mathrm{O}(48)-\mathrm{W}(2)-\mathrm{O}(41)$ & $91.7(7)$ & $\mathrm{O}(52)-\mathrm{W}(11)-\mathrm{O}(19)$ & $82.4(5)$ & $\mathrm{O}(39)-\mathrm{Gd}(2)-\mathrm{O}(78)$ & $71.6(5)$ \\
\hline $\mathrm{O}(18)-\mathrm{W}(2)-\mathrm{O}(41)$ & $158.5(6)$ & $\mathrm{O}(46)-\mathrm{W}(11)-\mathrm{O}(19)$ & $73.1(5)$ & $\mathrm{O}(76)-\mathrm{Gd}(2)-\mathrm{O}(78)$ & $130.5(6)$ \\
\hline $\mathrm{O}(25)-\mathrm{W}(2)-\mathrm{O}(50)$ & $98.0(7)$ & $\mathrm{O}(35)-\mathrm{W}(11)-\mathrm{O}(19)$ & $84.1(5)$ & $\mathrm{O}(74)-\mathrm{Gd}(2)-\mathrm{O}(78)$ & $66.3(5)$ \\
\hline $\mathrm{O}(48)-\mathrm{W}(2)-\mathrm{O}(50)$ & $160.5(6)$ & $\mathrm{O}(48)-\mathrm{W}(11)-\mathrm{O}(19)$ & $83.8(5)$ & $\mathrm{O}(69)-\mathrm{Gd}(2)-\mathrm{O}(78)$ & $124.2(6)$ \\
\hline $\mathrm{O}(18)-\mathrm{W}(2)-\mathrm{O}(50)$ & $83.8(7)$ & $\mathrm{O}(75)-\mathrm{W}(12)-\mathrm{O}(51)$ & $100.6(7)$ & $\mathrm{O}(73)-\mathrm{Gd}(2)-\mathrm{O}(78)$ & $129.6(6)$ \\
\hline $\mathrm{O}(41)-\mathrm{W}(2)-\mathrm{O}(50)$ & $88.1(7)$ & $\mathrm{O}(75)-\mathrm{W}(12)-\mathrm{O}(12)$ & $102.1(6)$ & $\mathrm{O}(77)-\mathrm{Gd}(2)-\mathrm{O}(78)$ & $122.8(5)$ \\
\hline $\mathrm{O}(25)-\mathrm{W}(2)-\mathrm{O}(7)$ & $172.6(7)$ & $\mathrm{O}(51)-\mathrm{W}(12)-\mathrm{O}(12)$ & $94.8(6)$ & $\mathrm{O}(11)-\mathrm{Gd}(2)-\mathrm{O}(78)$ & $68.1(5)$ \\
\hline $\mathrm{O}(48)-\mathrm{W}(2)-\mathrm{O}(7)$ & $80.4(6)$ & $\mathrm{O}(75)-\mathrm{W}(12)-\mathrm{O}(21)$ & $100.5(6)$ & $\mathrm{O}(42)-\mathrm{Mn}(1)-\mathrm{O}(47)$ & $93.1(6)$ \\
\hline $\mathrm{O}(18)-\mathrm{W}(2)-\mathrm{O}(7)$ & $86.2(5)$ & $\mathrm{O}(51)-\mathrm{W}(12)-\mathrm{O}(21)$ & $92.5(6)$ & $\mathrm{O}(42)-\mathrm{Mn}(1)-\mathrm{O}(52)^{\mathrm{i}}$ & $169.4(5)$ \\
\hline $\mathrm{O}(41)-\mathrm{W}(2)-\mathrm{O}(7)$ & $72.9(5)$ & $\mathrm{O}(12)-\mathrm{W}(12)-\mathrm{O}(21)$ & $154.5(6)$ & $\mathrm{O}(47)-\mathrm{Mn}(1)-\mathrm{O}(52)^{\mathrm{i}}$ & $94.3(6)$ \\
\hline $\mathrm{O}(50)-\mathrm{W}(2)-\mathrm{O}(7)$ & $80.9(6)$ & $\mathrm{O}(75)-\mathrm{W}(12)-\mathrm{O}(53)$ & $92.7(7)$ & $\mathrm{O}(42)-\mathrm{Mn}(1)-\mathrm{O}(32)^{\mathrm{i}}$ & $90.2(5)$ \\
\hline $\mathrm{O}(31)-\mathrm{W}(3)-\mathrm{O}(43)$ & $100.3(7)$ & $\mathrm{O}(51)-\mathrm{W}(12)-\mathrm{O}(53)$ & $166.6(6)$ & $\mathrm{O}(47)-\mathrm{Mn}(1)-\mathrm{O}(32)^{\mathrm{i}}$ & $173.4(5)$ \\
\hline $\mathrm{O}(31)-\mathrm{W}(3)-\mathrm{O}(15)$ & $102.8(6)$ & $\mathrm{O}(12)-\mathrm{W}(12)-\mathrm{O}(53)$ & $83.6(6)$ & $\mathrm{O}(52)^{\mathrm{i}}-\mathrm{Mn}(1)-\mathrm{O}(32)^{\mathrm{i}}$ & $81.7(5)$ \\
\hline $\mathrm{O}(43)-\mathrm{W}(3)-\mathrm{O}(15)$ & $95.8(7)$ & $\mathrm{O}(21)-\mathrm{W}(12)-\mathrm{O}(53)$ & $83.7(6)$ & $\mathrm{O}(42)-\mathrm{Mn}(1)-\mathrm{O}(49)$ & $91.3(6)$ \\
\hline $\mathrm{O}(31)-\mathrm{W}(3)-\mathrm{O}(46)$ & $98.7(6)$ & $\mathrm{O}(75)-\mathrm{W}(12)-\mathrm{O}(8)$ & $171.7(6)$ & $\mathrm{O}(47)-\mathrm{Mn}(1)-\mathrm{O}(49)$ & $91.2(5)$ \\
\hline $\mathrm{O}(43)-\mathrm{W}(3)-\mathrm{O}(46)$ & $91.2(6)$ & $\mathrm{O}(51)-\mathrm{W}(12)-\mathrm{O}(8)$ & $84.2(6)$ & $\mathrm{O}(52)^{\mathrm{i}}-\mathrm{Mn}(1)-\mathrm{O}(49)$ & $96.1(6)$ \\
\hline $\mathrm{O}(15)-\mathrm{W}(3)-\mathrm{O}(46)$ & $155.7(5)$ & $\mathrm{O}(12)-\mathrm{W}(12)-\mathrm{O}(8)$ & $84.1(5)$ & $\mathrm{O}(32)^{\mathrm{i}}-\mathrm{Mn}(1)-\mathrm{O}(49)$ & $94.5(5)$ \\
\hline $\mathrm{O}(31)-\mathrm{W}(3)-\mathrm{O}(55)$ & 93.1(7) & $\mathrm{O}(21)-\mathrm{W}(12)-\mathrm{O}(8)$ & $72.4(5)$ & $\mathrm{O}(42)-\mathrm{Mn}(1)-\mathrm{O}(58)$ & $87.7(6)$ \\
\hline
\end{tabular}




\begin{tabular}{|c|c|c|c|c|c|}
\hline $\mathrm{O}(43)-\mathrm{W}(3)-\mathrm{O}(55)$ & $166.0(6)$ & $\mathrm{O}(53)-\mathrm{W}(12)-\mathrm{O}(8)$ & $82.4(5)$ & $\mathrm{O}(47)-\mathrm{Mn}(1)-\mathrm{O}(58)$ & $91.4(5)$ \\
\hline $\mathrm{O}(15)-\mathrm{W}(3)-\mathrm{O}(55)$ & $85.1(6)$ & $\mathrm{O}(6)-\mathrm{W}(13)-\mathrm{O}(28)$ & $104.3(7)$ & $\mathrm{O}(52)^{\mathrm{i}}-\mathrm{Mn}(1)-\mathrm{O}(58)$ & $84.6(5)$ \\
\hline $\mathrm{O}(46)-\mathrm{W}(3)-\mathrm{O}(55)$ & $82.7(6)$ & $\mathrm{O}(6)-\mathrm{W}(13)-\mathrm{O}(34)$ & $102.7(8)$ & $\mathrm{O}(32)^{\mathrm{i}}-\mathrm{Mn}(1)-\mathrm{O}(58)$ & $82.9(5)$ \\
\hline $\mathrm{O}(31)-\mathrm{W}(3)-\mathrm{O}(19)$ & $170.8(6)$ & $\mathrm{O}(28)-\mathrm{W}(13)-\mathrm{O}(34)$ & $89.8(7)$ & $\mathrm{O}(49)-\mathrm{Mn}(1)-\mathrm{O}(58)$ & $177.2(5)$ \\
\hline $\mathrm{O}(43)-\mathrm{W}(3)-\mathrm{O}(19)$ & $84.7(6)$ & $\mathrm{O}(6)-\mathrm{W}(13)-\mathrm{O}(1)$ & $114.0(7)$ & $\mathrm{O}(51)-\mathrm{Mn}(2)-\mathrm{O}(43)^{\mathrm{i}}$ & $94.9(6)$ \\
\hline $\mathrm{O}(15)-\mathrm{W}(3)-\mathrm{O}(19)$ & $84.1(5)$ & $\mathrm{O}(28)-\mathrm{W}(13)-\mathrm{O}(1)$ & $72.4(6)$ & $\mathrm{O}(51)-\mathrm{Mn}(2)-\mathrm{O}(52)^{\mathrm{i}}$ & $92.0(5)$ \\
\hline $\mathrm{O}(46)-\mathrm{W}(3)-\mathrm{O}(19)$ & $73.3(5)$ & $\mathrm{O}(34)-\mathrm{W}(13)-\mathrm{O}(1)$ & $142.0(6)$ & $\mathrm{O}(43)^{\mathrm{i}}-\mathrm{Mn}(2)-\mathrm{O}(52)^{\mathrm{i}}$ & $95.2(6)$ \\
\hline $\mathrm{O}(55)-\mathrm{W}(3)-\mathrm{O}(19)$ & $81.4(5)$ & $\mathrm{O}(6)-\mathrm{W}(13)-\mathrm{O}(5)$ & $108.4(7)$ & $\mathrm{O}(51)-\mathrm{Mn}(2)-\mathrm{O}(32)$ & $96.0(5)$ \\
\hline $\mathrm{O}(17)-\mathrm{W}(4)-\mathrm{O}(47)$ & $99.9(7)$ & $\mathrm{O}(28)-\mathrm{W}(13)-\mathrm{O}(5)$ & $144.8(7)$ & $\mathrm{O}(43)^{\mathrm{i}}-\mathrm{Mn}(2)-\mathrm{O}(32)$ & $94.6(6)$ \\
\hline $\mathrm{O}(17)-\mathrm{W}(4)-\mathrm{O}(12)$ & $101.0(6)$ & $\mathrm{O}(34)-\mathrm{W}(13)-\mathrm{O}(5)$ & $70.9(6)$ & $\mathrm{O}(52)^{\mathrm{i}}-\mathrm{Mn}(2)-\mathrm{O}(32)$ & $166.8(5)$ \\
\hline $\mathrm{O}(47)-\mathrm{W}(4)-\mathrm{O}(12)$ & $95.9(7)$ & $\mathrm{O}(1)-\mathrm{W}(13)-\mathrm{O}(5)$ & $104.8(5)$ & $\mathrm{O}(51)-\mathrm{Mn}(2)-\mathrm{O}(58)^{\mathrm{i}}$ & $172.8(6)$ \\
\hline $\mathrm{O}(17)-\mathrm{W}(4)-\mathrm{O}(45)$ & $100.4(6)$ & $\mathrm{O}(6)-\mathrm{W}(13)-\mathrm{O}(40)$ & $169.4(7)$ & $\mathrm{O}(43)^{\mathrm{i}}-\mathrm{Mn}(2)-\mathrm{O}(58)^{\mathrm{i}}$ & $92.3(6)$ \\
\hline $\mathrm{O}(47)-\mathrm{W}(4)-\mathrm{O}(45)$ & $93.5(6)$ & $\mathrm{O}(28)-\mathrm{W}(13)-\mathrm{O}(40)$ & $84.7(6)$ & $\mathrm{O}(52)^{\mathrm{i}}-\mathrm{Mn}(2)-\mathrm{O}(58)^{\mathrm{i}}$ & $87.1(5)$ \\
\hline $\mathrm{O}(12)-\mathrm{W}(4)-\mathrm{O}(45)$ & $154.8(6)$ & $\mathrm{O}(34)-\mathrm{W}(13)-\mathrm{O}(40)$ & $82.6(6)$ & $\mathrm{O}(32)-\mathrm{Mn}(2)-\mathrm{O}(58)^{\mathrm{i}}$ & $83.7(5)$ \\
\hline $\mathrm{O}(17)-\mathrm{W}(4)-\mathrm{O}(56)$ & $92.9(7)$ & $\mathrm{O}(1)-\mathrm{W}(13)-\mathrm{O}(40)$ & $63.0(6)$ & $\mathrm{O}(51)-\mathrm{Mn}(2)-\mathrm{O}(58)$ & $88.1(6)$ \\
\hline $\mathrm{O}(47)-\mathrm{W}(4)-\mathrm{O}(56)$ & $166.7(6)$ & $\mathrm{O}(5)-\mathrm{W}(13)-\mathrm{O}(40)$ & $64.3(5)$ & $\mathrm{O}(43)^{\mathrm{i}}-\mathrm{Mn}(2)-\mathrm{O}(58)$ & $177.0(6)$ \\
\hline $\mathrm{O}(12)-\mathrm{W}(4)-\mathrm{O}(56)$ & $85.0(6)$ & $\mathrm{O}(10)-\mathrm{W}(14)-\mathrm{O}(5)$ & $105.2(7)$ & $\mathrm{O}(52)^{\mathrm{i}}-\mathrm{Mn}(2)-\mathrm{O}(58)$ & $84.4(5)$ \\
\hline $\mathrm{O}(45)-\mathrm{W}(4)-\mathrm{O}(56)$ & $80.7(6)$ & $\mathrm{O}(10)-\mathrm{W}(14)-\mathrm{O}(44)$ & $102.1(8)$ & $\mathrm{O}(32)-\mathrm{Mn}(2)-\mathrm{O}(58)$ & $85.4(5)$ \\
\hline $\mathrm{O}(17)-\mathrm{W}(4)-\mathrm{O}(20)$ & $172.0(6)$ & $\mathrm{O}(5)-\mathrm{W}(14)-\mathrm{O}(44)$ & $71.1(6)$ & $\mathrm{O}(58)^{\mathrm{i}}-\mathrm{Mn}(2)-\mathrm{O}(58)$ & $84.7(6)$ \\
\hline $\mathrm{O}(47)-\mathrm{W}(4)-\mathrm{O}(20)$ & $86.4(6)$ & $\mathrm{O}(10)-\mathrm{W}(14)-\mathrm{O}(50)$ & $105.4(8)$ & $\mathrm{O}(65)-\mathrm{Mn}(3)-\mathrm{O}(61)$ & $174.1(11)$ \\
\hline $\mathrm{O}(12)-\mathrm{W}(4)-\mathrm{O}(20)$ & $83.2(5)$ & $\mathrm{O}(5)-\mathrm{W}(14)-\mathrm{O}(50)$ & $145.5(7)$ & $\mathrm{O}(65)-\mathrm{Mn}(3)-\mathrm{O}(60)$ & $92.4(11)$ \\
\hline $\mathrm{O}(45)-\mathrm{W}(4)-\mathrm{O}(20)$ & $74.1(5)$ & $\mathrm{O}(44)-\mathrm{W}(14)-\mathrm{O}(50)$ & $87.2(7)$ & $\mathrm{O}(61)-\mathrm{Mn}(3)-\mathrm{O}(60)$ & $91.7(11)$ \\
\hline $\mathrm{O}(56)-\mathrm{W}(4)-\mathrm{O}(20)$ & $80.6(5)$ & $\mathrm{O}(10)-\mathrm{W}(14)-\mathrm{O}(4)$ & $103.8(9)$ & $\mathrm{O}(65)-\mathrm{Mn}(3)-\mathrm{O}(59)$ & $89.3(13)$ \\
\hline $\mathrm{O}(2)-\mathrm{W}(5)-\mathrm{O}(55)$ & $103.3(7)$ & $\mathrm{O}(5)-\mathrm{W}(14)-\mathrm{O}(4)$ & $106.4(7)$ & $\mathrm{O}(61)-\mathrm{Mn}(3)-\mathrm{O}(59)$ & $86.2(14)$ \\
\hline $\mathrm{O}(2)-\mathrm{W}(5)-\mathrm{O}(27)$ & $101.7(7)$ & $\mathrm{O}(44)-\mathrm{W}(14)-\mathrm{O}(4)$ & $153.7(8)$ & $\mathrm{O}(60)-\mathrm{Mn}(3)-\mathrm{O}(59)$ & $175.1(12)$ \\
\hline $\mathrm{O}(55)-\mathrm{W}(5)-\mathrm{O}(27)$ & $89.8(7)$ & $\mathrm{O}(50)-\mathrm{W}(14)-\mathrm{O}(4)$ & $81.3(8)$ & $\mathrm{O}(65)-\mathrm{Mn}(3)-\mathrm{O}(26)$ & 91.1(9) \\
\hline $\mathrm{O}(2)-\mathrm{W}(5)-\mathrm{O}(41)$ & $99.0(7)$ & $\mathrm{O}(10)-\mathrm{W}(14)-\mathrm{O}(40)$ & $169.3(8)$ & $\mathrm{O}(61)-\mathrm{Mn}(3)-\mathrm{O}(26)$ & $93.2(9)$ \\
\hline $\mathrm{O}(55)-\mathrm{W}(5)-\mathrm{O}(41)$ & $93.9(7)$ & $\mathrm{O}(5)-\mathrm{W}(14)-\mathrm{O}(40)$ & $68.6(5)$ & $\mathrm{O}(60)-\mathrm{Mn}(3)-\mathrm{O}(26)$ & $87.3(8)$ \\
\hline $\mathrm{O}(27)-\mathrm{W}(5)-\mathrm{O}(41)$ & $157.5(6)$ & $\mathrm{O}(44)-\mathrm{W}(14)-\mathrm{O}(40)$ & $84.3(6)$ & $\mathrm{O}(59)-\mathrm{Mn}(3)-\mathrm{O}(26)$ & $97.2(10)$ \\
\hline $\mathrm{O}(2)-\mathrm{W}(5)-\mathrm{O}(38)$ & $94.9(8)$ & $\mathrm{O}(50)-\mathrm{W}(14)-\mathrm{O}(40)$ & $83.3(6)$ & $\mathrm{O}(65)-\mathrm{Mn}(3)-\mathrm{O}(66)$ & $87.1(10)$ \\
\hline $\mathrm{O}(55)-\mathrm{W}(5)-\mathrm{O}(38)$ & $161.5(6)$ & $\mathrm{O}(4)-\mathrm{W}(14)-\mathrm{O}(40)$ & $70.9(7)$ & $\mathrm{O}(61)-\mathrm{Mn}(3)-\mathrm{O}(66)$ & $89.1(10)$ \\
\hline $\mathrm{O}(27)-\mathrm{W}(5)-\mathrm{O}(38)$ & $83.1(7)$ & $\mathrm{O}(13)-\mathrm{W}(15)-\mathrm{O}(1)$ & $107.8(7)$ & $\mathrm{O}(60)-\mathrm{Mn}(3)-\mathrm{O}(66)$ & $86.1(9)$ \\
\hline $\mathrm{O}(41)-\mathrm{W}(5)-\mathrm{O}(38)$ & $86.5(7)$ & $\mathrm{O}(13)-\mathrm{W}(15)-\mathrm{O}(14)$ & $102.9(8)$ & $\mathrm{O}(59)-\mathrm{Mn}(3)-\mathrm{O}(66)$ & $89.5(11)$ \\
\hline $\mathrm{O}(2)-\mathrm{W}(5)-\mathrm{O}(7)$ & $171.2(6)$ & $\mathrm{O}(1)-\mathrm{W}(15)-\mathrm{O}(14)$ & $72.9(6)$ & $\mathrm{O}(26)-\mathrm{Mn}(3)-\mathrm{O}(66)$ & $173.1(7)$ \\
\hline $\mathrm{O}(55)-\mathrm{W}(5)-\mathrm{O}(7)$ & $81.6(6)$ & $\mathrm{O}(13)-\mathrm{W}(15)-\mathrm{O}(38)$ & $103.6(7)$ & $\mathrm{W}(15)-\mathrm{O}(1)-\mathrm{W}(13)$ & $132.5(7)$ \\
\hline $\mathrm{O}(27)-\mathrm{W}(5)-\mathrm{O}(7)$ & $85.6(5)$ & $\mathrm{O}(1)-\mathrm{W}(15)-\mathrm{O}(38)$ & $146.7(7)$ & $\mathrm{W}(14)-\mathrm{O}(4)-\mathrm{W}(15)$ & $125.1(11)$ \\
\hline $\mathrm{O}(41)-\mathrm{W}(5)-\mathrm{O}(7)$ & $73.1(5)$ & $\mathrm{O}(14)-\mathrm{W}(15)-\mathrm{O}(38)$ & $89.7(7)$ & $\mathrm{W}(14)-\mathrm{O}(5)-\mathrm{W}(13)$ & $126.7(7)$ \\
\hline $\mathrm{O}(38)-\mathrm{W}(5)-\mathrm{O}(7)$ & $80.9(6)$ & $\mathrm{O}(13)-\mathrm{W}(15)-\mathrm{O}(4)$ & 101.2(9) & $\mathrm{P}(1)-\mathrm{O}(7)-\mathrm{W}(5)$ & 129.1(8) \\
\hline $\mathrm{O}(39)-\mathrm{W}(6)-\mathrm{O}(32)$ & $100.3(7)$ & $\mathrm{O}(1)-\mathrm{W}(15)-\mathrm{O}(4)$ & $102.7(7)$ & $\mathrm{P}(1)-\mathrm{O}(7)-\mathrm{W}(2)$ & $127.3(8)$ \\
\hline $\mathrm{O}(39)-\mathrm{W}(6)-\mathrm{O}(35)$ & $101.2(6)$ & $\mathrm{O}(14)-\mathrm{W}(15)-\mathrm{O}(4)$ & $155.6(9)$ & $\mathrm{W}(5)-\mathrm{O}(7)-\mathrm{W}(2)$ & $90.7(4)$ \\
\hline $\mathrm{O}(32)-\mathrm{W}(6)-\mathrm{O}(35)$ & $92.3(6)$ & $\mathrm{O}(38)-\mathrm{W}(15)-\mathrm{O}(4)$ & $81.4(7)$ & $\mathrm{P}(2)-\mathrm{O}(8)-\mathrm{W}(6)$ & $128.2(8)$ \\
\hline
\end{tabular}




\begin{tabular}{|c|c|c|c|c|c|}
\hline $\mathrm{O}(39)-\mathrm{W}(6)-\mathrm{O}(21)$ & $99.6(6)$ & $\mathrm{O}(13)-\mathrm{W}(15)-\mathrm{O}(40)$ & $167.3(7)$ & $\mathrm{P}(2)-\mathrm{O}(8)-\mathrm{W}(12)$ & $127.2(7)$ \\
\hline $\mathrm{O}(32)-\mathrm{W}(6)-\mathrm{O}(21)$ & $95.0(6)$ & $\mathrm{O}(1)-\mathrm{W}(15)-\mathrm{O}(40)$ & $66.1(6)$ & $\mathrm{W}(6)-\mathrm{O}(8)-\mathrm{W}(12)$ & $91.6(5)$ \\
\hline $\mathrm{O}(35)-\mathrm{W}(6)-\mathrm{O}(21)$ & $156.4(6)$ & $\mathrm{O}(14)-\mathrm{W}(15)-\mathrm{O}(40)$ & $86.2(6)$ & $\mathrm{W}(4)-\mathrm{O}(12)-\mathrm{W}(12)$ & $148.0(9)$ \\
\hline $\mathrm{O}(39)-\mathrm{W}(6)-\mathrm{O}(57)$ & $91.9(6)$ & $\mathrm{O}(38)-\mathrm{W}(15)-\mathrm{O}(40)$ & $85.0(6)$ & $\mathrm{W}(15)-\mathrm{O}(14)-\mathrm{W}(1)$ & $148.5(9)$ \\
\hline $\mathrm{O}(32)-\mathrm{W}(6)-\mathrm{O}(57)$ & $167.7(6)$ & $\mathrm{O}(4)-\mathrm{W}(15)-\mathrm{O}(40)$ & $70.5(7)$ & $\mathrm{W}(3)-\mathrm{O}(15)-\mathrm{W}(9)$ & $148.3(9)$ \\
\hline $\mathrm{O}(35)-\mathrm{W}(6)-\mathrm{O}(57)$ & $84.1(6)$ & $\mathrm{O}(37)-\mathrm{P}(1)-\mathrm{O}(36)$ & $112.5(8)$ & $\mathrm{W}(2)-\mathrm{O}(18)-\mathrm{W}(7)$ & $154.4(10)$ \\
\hline $\mathrm{O}(21)-\mathrm{W}(6)-\mathrm{O}(57)$ & $84.1(6)$ & $\mathrm{O}(37)-\mathrm{P}(1)-\mathrm{O}(7)$ & $109.9(8)$ & $\mathrm{P}(2)-\mathrm{O}(19)-\mathrm{W}(3)$ & $129.0(7)$ \\
\hline $\mathrm{O}(39)-\mathrm{W}(6)-\mathrm{O}(8)$ & $172.3(5)$ & $\mathrm{O}(36)-\mathrm{P}(1)-\mathrm{O}(7)$ & $111.2(8)$ & $\mathrm{P}(2)-\mathrm{O}(19)-\mathrm{W}(11)$ & $128.1(8)$ \\
\hline $\mathrm{O}(32)-\mathrm{W}(6)-\mathrm{O}(8)$ & $84.1(6)$ & $\mathrm{O}(37)-\mathrm{P}(1)-\mathrm{O}(40)$ & 108.7(9) & $\mathrm{W}(3)-\mathrm{O}(19)-\mathrm{W}(11)$ & $91.2(4)$ \\
\hline $\mathrm{O}(35)-\mathrm{W}(6)-\mathrm{O}(8)$ & $84.7(5)$ & $\mathrm{O}(36)-\mathrm{P}(1)-\mathrm{O}(40)$ & $107.1(8)$ & $\mathrm{P}(2)-\mathrm{O}(20)-\mathrm{W}(4)$ & $129.5(8)$ \\
\hline $\mathrm{O}(21)-\mathrm{W}(6)-\mathrm{O}(8)$ & $73.7(5)$ & $\mathrm{O}(7)-\mathrm{P}(1)-\mathrm{O}(40)$ & $107.2(8)$ & $\mathrm{P}(2)-\mathrm{O}(20)-\mathrm{W}(9)$ & $126.1(7)$ \\
\hline $\mathrm{O}(57)-\mathrm{W}(6)-\mathrm{O}(8)$ & $83.8(5)$ & $\mathrm{O}(58)-\mathrm{P}(2)-\mathrm{O}(20)$ & $109.0(8)$ & $\mathrm{W}(4)-\mathrm{O}(20)-\mathrm{W}(9)$ & $90.6(4)$ \\
\hline $\mathrm{O}(22)-\mathrm{W}(7)-\mathrm{O}(57)$ & $100.3(8)$ & $\mathrm{O}(58)-\mathrm{P}(2)-\mathrm{O}(19)$ & $109.0(8)$ & $\mathrm{W}(6)-\mathrm{O}(21)-\mathrm{W}(12)$ & $121.8(7)$ \\
\hline $\mathrm{O}(22)-\mathrm{W}(7)-\mathrm{O}(18)$ & $102.0(7)$ & $\mathrm{O}(20)-\mathrm{P}(2)-\mathrm{O}(19)$ & $110.8(8)$ & $\mathrm{W}(8)-\mathrm{O}(23)-\mathrm{W}(10)$ & $155.4(8)$ \\
\hline $\mathrm{O}(57)-\mathrm{W}(7)-\mathrm{O}(18)$ & $90.5(7)$ & $\mathrm{O}(58)-\mathrm{P}(2)-\mathrm{O}(8)$ & $107.5(8)$ & $\mathrm{W}(10)-\mathrm{O}(24)-\mathrm{W}(1)$ & $120.1(7)$ \\
\hline $\mathrm{O}(22)-\mathrm{W}(7)-\mathrm{O}(30)$ & $98.8(6)$ & $\mathrm{O}(20)-\mathrm{P}(2)-\mathrm{O}(8)$ & $109.4(8)$ & $\mathrm{W}(1)-\mathrm{O}(26)-\mathrm{Mn}(3)$ & $153.9(10)$ \\
\hline $\mathrm{O}(57)-\mathrm{W}(7)-\mathrm{O}(30)$ & $93.0(6)$ & $\mathrm{O}(19)-\mathrm{P}(2)-\mathrm{O}(8)$ & $111.0(7)$ & $\mathrm{W}(1)-\mathrm{O}(27)-\mathrm{W}(5)$ & $156.1(8)$ \\
\hline $\mathrm{O}(18)-\mathrm{W}(7)-\mathrm{O}(30)$ & $157.9(6)$ & $\mathrm{O}(70)-\mathrm{Gd}(1)-\mathrm{O}(62)$ & $70.1(10)$ & $\mathrm{W}(13)-\mathrm{O}(28)-\mathrm{W}(10)$ & $147.7(10)$ \\
\hline $\mathrm{O}(22)-\mathrm{W}(7)-\mathrm{O}(44)$ & $97.5(8)$ & $\mathrm{O}(70)-\mathrm{Gd}(1)-\mathrm{O}(64)$ & $70.7(8)$ & $\mathrm{W}(8)-\mathrm{O}(30)-\mathrm{W}(7)$ & 121.1(7) \\
\hline $\mathrm{O}(57)-\mathrm{W}(7)-\mathrm{O}(44)$ & $162.1(6)$ & $\mathrm{O}(62)-\mathrm{Gd}(1)-\mathrm{O}(64)$ & $78.0(11)$ & $\mathrm{W}(6)-\mathrm{O}(32)-\mathrm{Mn}(2)$ & $131.8(7)$ \\
\hline $\mathrm{O}(18)-\mathrm{W}(7)-\mathrm{O}(44)$ & $83.3(7)$ & $\mathrm{O}(70)-\mathrm{Gd}(1)-\mathrm{O}(67)$ & $139.7(8)$ & $\mathrm{W}(6)-\mathrm{O}(32)-\mathrm{Mn}(1)^{\mathrm{i}}$ & $127.4(7)$ \\
\hline $\mathrm{O}(30)-\mathrm{W}(7)-\mathrm{O}(44)$ & $86.8(6)$ & $\mathrm{O}(62)-\mathrm{Gd}(1)-\mathrm{O}(67)$ & $70.8(10)$ & $\operatorname{Mn}(2)-O(32)-\operatorname{Mn}(1)^{i}$ & $98.6(6)$ \\
\hline $\mathrm{O}(22)-\mathrm{W}(7)-\mathrm{O}(36)$ & $172.2(6)$ & $\mathrm{O}(64)-\mathrm{Gd}(1)-\mathrm{O}(67)$ & $91.8(8)$ & $\mathrm{W}(10)-\mathrm{O}(33)-\mathrm{Gd}(1)$ & $174.4(10)$ \\
\hline $\mathrm{O}(57)-\mathrm{W}(7)-\mathrm{O}(36)$ & $81.7(6)$ & $\mathrm{O}(70)-\mathrm{Gd}(1)-\mathrm{O}(71)$ & $139.4(7)$ & $\mathrm{W}(8)-\mathrm{O}(34)-\mathrm{W}(13)$ & 149.4(9) \\
\hline $\mathrm{O}(18)-\mathrm{W}(7)-\mathrm{O}(36)$ & $85.4(5)$ & $\mathrm{O}(62)-\mathrm{Gd}(1)-\mathrm{O}(71)$ & $136.3(10)$ & $\mathrm{W}(6)-\mathrm{O}(35)-\mathrm{W}(11)$ & $147.4(8)$ \\
\hline $\mathrm{O}(30)-\mathrm{W}(7)-\mathrm{O}(36)$ & $73.5(5)$ & $\mathrm{O}(64)-\mathrm{Gd}(1)-\mathrm{O}(71)$ & $134.3(7)$ & $\mathrm{P}(1)-\mathrm{O}(36)-\mathrm{W}(7)$ & $128.8(8)$ \\
\hline $\mathrm{O}(44)-\mathrm{W}(7)-\mathrm{O}(36)$ & $81.1(6)$ & $\mathrm{O}(67)-\mathrm{Gd}(1)-\mathrm{O}(71)$ & $78.5(7)$ & $\mathrm{P}(1)-\mathrm{O}(36)-\mathrm{W}(8)$ & $127.5(8)$ \\
\hline $\mathrm{O}(16)-\mathrm{W}(8)-\mathrm{O}(53)$ & $100.3(8)$ & $\mathrm{O}(70)-\mathrm{Gd}(1)-\mathrm{O}(72)$ & $78.6(7)$ & $\mathrm{W}(7)-\mathrm{O}(36)-\mathrm{W}(8)$ & $90.9(5)$ \\
\hline $\mathrm{O}(16)-\mathrm{W}(8)-\mathrm{O}(23)$ & $102.1(6)$ & $\mathrm{O}(62)-\mathrm{Gd}(1)-\mathrm{O}(72)$ & $145.5(10)$ & $\mathrm{P}(1)-\mathrm{O}(37)-\mathrm{W}(10)$ & $127.4(8)$ \\
\hline $\mathrm{O}(53)-\mathrm{W}(8)-\mathrm{O}(23)$ & $90.2(7)$ & $\mathrm{O}(64)-\mathrm{Gd}(1)-\mathrm{O}(72)$ & $78.3(8)$ & $\mathrm{P}(1)-\mathrm{O}(37)-\mathrm{W}(1)$ & $129.6(9)$ \\
\hline $\mathrm{O}(16)-\mathrm{W}(8)-\mathrm{O}(30)$ & $99.1(6)$ & $\mathrm{O}(67)-\mathrm{Gd}(1)-\mathrm{O}(72)$ & $134.6(6)$ & $\mathrm{W}(10)-\mathrm{O}(37)-\mathrm{W}(1)$ & $89.9(5)$ \\
\hline $\mathrm{O}(53)-\mathrm{W}(8)-\mathrm{O}(30)$ & 93.1(7) & $\mathrm{O}(71)-\mathrm{Gd}(1)-\mathrm{O}(72)$ & $77.7(7)$ & $\mathrm{W}(15)-\mathrm{O}(38)-\mathrm{W}(5)$ & 147.6(9) \\
\hline $\mathrm{O}(23)-\mathrm{W}(8)-\mathrm{O}(30)$ & $157.6(6)$ & $\mathrm{O}(70)-\mathrm{Gd}(1)-\mathrm{O}(63)$ & $102.9(8)$ & $\mathrm{W}(6)-\mathrm{O}(39)-\mathrm{Gd}(2)$ & $157.5(7)$ \\
\hline $\mathrm{O}(16)-\mathrm{W}(8)-\mathrm{O}(34)$ & $97.9(8)$ & $\mathrm{O}(62)-\mathrm{Gd}(1)-\mathrm{O}(63)$ & $67.3(11)$ & $\mathrm{P}(1)-\mathrm{O}(40)-\mathrm{W}(14)$ & $125.2(8)$ \\
\hline $\mathrm{O}(53)-\mathrm{W}(8)-\mathrm{O}(34)$ & $161.7(6)$ & $\mathrm{O}(64)-\mathrm{Gd}(1)-\mathrm{O}(63)$ & $144.5(9)$ & $\mathrm{P}(1)-\mathrm{O}(40)-\mathrm{W}(15)$ & $123.0(8)$ \\
\hline $\mathrm{O}(23)-\mathrm{W}(8)-\mathrm{O}(34)$ & $84.4(7)$ & $\mathrm{O}(67)-\mathrm{Gd}(1)-\mathrm{O}(63)$ & $70.4(7)$ & $\mathrm{W}(14)-\mathrm{O}(40)-\mathrm{W}(15)$ & $92.5(6)$ \\
\hline $\mathrm{O}(30)-\mathrm{W}(8)-\mathrm{O}(34)$ & $85.5(7)$ & $\mathrm{O}(71)-\mathrm{Gd}(1)-\mathrm{O}(63)$ & $73.5(8)$ & $\mathrm{P}(1)-\mathrm{O}(40)-\mathrm{W}(13)$ & $123.3(7)$ \\
\hline $\mathrm{O}(16)-\mathrm{W}(8)-\mathrm{O}(36)$ & $172.7(6)$ & $\mathrm{O}(72)-\mathrm{Gd}(1)-\mathrm{O}(63)$ & $136.0(8)$ & $\mathrm{W}(14)-\mathrm{O}(40)-\mathrm{W}(13)$ & $91.9(5)$ \\
\hline $\mathrm{O}(53)-\mathrm{W}(8)-\mathrm{O}(36)$ & $81.7(6)$ & $\mathrm{O}(70)-\mathrm{Gd}(1)-\mathrm{O}(33)$ & $129.0(6)$ & $\mathrm{W}(15)-\mathrm{O}(40)-\mathrm{W}(13)$ & $91.6(6)$ \\
\hline $\mathrm{O}(23)-\mathrm{W}(8)-\mathrm{O}(36)$ & $84.9(5)$ & $\mathrm{O}(62)-\mathrm{Gd}(1)-\mathrm{O}(33)$ & $122.3(9)$ & $\mathrm{W}(2)-\mathrm{O}(41)-\mathrm{W}(5)$ & $122.9(7)$ \\
\hline $\mathrm{O}(30)-\mathrm{W}(8)-\mathrm{O}(36)$ & $73.7(5)$ & $\mathrm{O}(64)-\mathrm{Gd}(1)-\mathrm{O}(33)$ & $65.7(7)$ & $\mathrm{W}(9)-\mathrm{O}(42)-\mathrm{Mn}(1)$ & $136.3(7)$ \\
\hline
\end{tabular}




\begin{tabular}{|c|c|c|c|c|c|}
\hline $\mathrm{O}(34)-\mathrm{W}(8)-\mathrm{O}(36)$ & $80.4(6)$ & $\mathrm{O}(67)-\mathrm{Gd}(1)-\mathrm{O}(33)$ & $67.4(6)$ & $\mathrm{W}(3)-\mathrm{O}(43)-\mathrm{Mn}(2)^{\mathrm{i}}$ & $135.0(8)$ \\
\hline $\mathrm{O}(29)-\mathrm{W}(9)-\mathrm{O}(42)$ & $101.1(7)$ & $\mathrm{O}(71)-\mathrm{Gd}(1)-\mathrm{O}(33)$ & $69.4(6)$ & $\mathrm{W}(14)-\mathrm{O}(44)-\mathrm{W}(7)$ & $150.7(8)$ \\
\hline $\mathrm{O}(29)-\mathrm{W}(9)-\mathrm{O}(45)$ & $99.2(6)$ & $\mathrm{O}(72)-\mathrm{Gd}(1)-\mathrm{O}(33)$ & $68.1(5)$ & $\mathrm{W}(4)-\mathrm{O}(45)-\mathrm{W}(9)$ & $120.8(6)$ \\
\hline $\mathrm{O}(42)-\mathrm{W}(9)-\mathrm{O}(45)$ & $94.5(6)$ & $\mathrm{O}(63)-\mathrm{Gd}(1)-\mathrm{O}(33)$ & $127.9(7)$ & $\mathrm{W}(11)-\mathrm{O}(46)-\mathrm{W}(3)$ & $122.0(6)$ \\
\hline $\mathrm{O}(29)-\mathrm{W}(9)-\mathrm{O}(15)$ & $102.0(6)$ & $\mathrm{O}(70)-\mathrm{Gd}(1)-\mathrm{O}(68)$ & $68.8(7)$ & $\mathrm{W}(4)-\mathrm{O}(47)-\mathrm{Mn}(1)$ & $133.5(8)$ \\
\hline $\mathrm{O}(42)-\mathrm{W}(9)-\mathrm{O}(15)$ & $95.2(6)$ & $\mathrm{O}(62)-\mathrm{Gd}(1)-\mathrm{O}(68)$ & $108.9(10)$ & $\mathrm{W}(2)-\mathrm{O}(48)-\mathrm{W}(11)$ & $167.7(8)$ \\
\hline $\mathrm{O}(45)-\mathrm{W}(9)-\mathrm{O}(15)$ & $154.5(5)$ & $\mathrm{O}(64)-\mathrm{Gd}(1)-\mathrm{O}(68)$ & $132.9(7)$ & $\mathrm{W}(14)-\mathrm{O}(50)-\mathrm{W}(2)$ & $150.3(9)$ \\
\hline $\mathrm{O}(29)-\mathrm{W}(9)-\mathrm{O}(54)$ & $92.6(7)$ & $\mathrm{O}(67)-\mathrm{Gd}(1)-\mathrm{O}(68)$ & $135.0(7)$ & $\mathrm{W}(12)-\mathrm{O}(51)-\mathrm{Mn}(2)$ & $135.4(7)$ \\
\hline $\mathrm{O}(42)-\mathrm{W}(9)-\mathrm{O}(54)$ & $166.3(6)$ & $\mathrm{O}(71)-\mathrm{Gd}(1)-\mathrm{O}(68)$ & $72.6(6)$ & $\mathrm{W}(11)-\mathrm{O}(52)-\mathrm{Mn}(1)^{\mathrm{i}}$ & $127.8(7)$ \\
\hline $\mathrm{O}(45)-\mathrm{W}(9)-\mathrm{O}(54)$ & $82.1(6)$ & $\mathrm{O}(72)-\mathrm{Gd}(1)-\mathrm{O}(68)$ & $71.2(6)$ & $\mathrm{W}(11)-\mathrm{O}(52)-\mathrm{Mn}(2)^{\mathrm{i}}$ & $131.9(7)$ \\
\hline $\mathrm{O}(15)-\mathrm{W}(9)-\mathrm{O}(54)$ & $82.9(6)$ & $\mathrm{O}(63)-\mathrm{Gd}(1)-\mathrm{O}(68)$ & $68.8(7)$ & $\operatorname{Mn}(1)^{\mathrm{i}}-\mathrm{O}(52)-\mathrm{Mn}(2)^{\mathrm{i}}$ & $98.2(6)$ \\
\hline $\mathrm{O}(29)-\mathrm{W}(9)-\mathrm{O}(20)$ & $171.6(6)$ & $\mathrm{O}(33)-\mathrm{Gd}(1)-\mathrm{O}(68)$ & $128.7(6)$ & $\mathrm{W}(8)-\mathrm{O}(53)-\mathrm{W}(12)$ & $165.1(9)$ \\
\hline $\mathrm{O}(42)-\mathrm{W}(9)-\mathrm{O}(20)$ & $83.6(6)$ & $\mathrm{O}(3)-\mathrm{Gd}(2)-\mathrm{O}(39)$ & $142.9(5)$ & $\mathrm{W}(1)-\mathrm{O}(54)-\mathrm{W}(9)$ & $166.1(7)$ \\
\hline $\mathrm{O}(45)-\mathrm{W}(9)-\mathrm{O}(20)$ & $73.3(5)$ & $\mathrm{O}(3)-\mathrm{Gd}(2)-\mathrm{O}(76)$ & $139.7(6)$ & $\mathrm{W}(5)-\mathrm{O}(55)-\mathrm{W}(3)$ & $166.5(8)$ \\
\hline $\mathrm{O}(15)-\mathrm{W}(9)-\mathrm{O}(20)$ & $84.4(5)$ & $\mathrm{O}(39)-\mathrm{Gd}(2)-\mathrm{O}(76)$ & $68.8(6)$ & $\mathrm{W}(10)-\mathrm{O}(56)-\mathrm{W}(4)$ & $163.0(8)$ \\
\hline $\mathrm{O}(54)-\mathrm{W}(9)-\mathrm{O}(20)$ & $82.7(5)$ & $\mathrm{O}(3)-\mathrm{Gd}(2)-\mathrm{O}(74)$ & $80.9(6)$ & $\mathrm{W}(7)-\mathrm{O}(57)-\mathrm{W}(6)$ & $164.9(7)$ \\
\hline $\mathrm{O}(33)-\mathrm{W}(10)-\mathrm{O}(56)$ & $100.9(7)$ & $\mathrm{O}(39)-\mathrm{Gd}(2)-\mathrm{O}(74)$ & $85.5(5)$ & $\mathrm{P}(2)-\mathrm{O}(58)-\mathrm{Mn}(2)^{\mathrm{i}}$ & $120.9(7)$ \\
\hline $\mathrm{O}(33)-\mathrm{W}(10)-\mathrm{O}(23)$ & $100.8(6)$ & $\mathrm{O}(76)-\mathrm{Gd}(2)-\mathrm{O}(74)$ & $136.3(6)$ & $\mathrm{P}(2)-\mathrm{O}(58)-\mathrm{Mn}(2)$ & $124.0(8)$ \\
\hline $\mathrm{O}(56)-\mathrm{W}(10)-\mathrm{O}(23)$ & $90.7(7)$ & $\mathrm{O}(3)-\mathrm{Gd}(2)-\mathrm{O}(69)$ & $132.7(6)$ & $\mathrm{Mn}(2)^{\mathrm{i}}-\mathrm{O}(58)-\mathrm{Mn}(2)$ & $95.3(6)$ \\
\hline $\mathrm{O}(33)-\mathrm{W}(10)-\mathrm{O}(24)$ & $99.2(6)$ & $\mathrm{O}(39)-\mathrm{Gd}(2)-\mathrm{O}(69)$ & $72.1(5)$ & $\mathrm{P}(2)-\mathrm{O}(58)-\mathrm{Mn}(1)$ & $122.7(8)$ \\
\hline $\mathrm{O}(56)-\mathrm{W}(10)-\mathrm{O}(24)$ & $92.7(6)$ & $\mathrm{O}(76)-\mathrm{Gd}(2)-\mathrm{O}(69)$ & $68.7(7)$ & $\mathrm{Mn}(2)^{\mathrm{i}}-\mathrm{O}(58)-\mathrm{Mn}(1)$ & $94.1(5)$ \\
\hline $\mathrm{O}(23)-\mathrm{W}(10)-\mathrm{O}(24)$ & $158.7(5)$ & $\mathrm{O}(74)-\mathrm{Gd}(2)-\mathrm{O}(69)$ & $70.0(7)$ & $\mathrm{Mn}(2)-\mathrm{O}(58)-\mathrm{Mn}(1)$ & $92.0(6)$ \\
\hline $\mathrm{O}(33)-\mathrm{W}(10)-\mathrm{O}(28)$ & $96.6(7)$ & & & & \\
\hline
\end{tabular}

Symmetry transformations used to generate equivalent atoms: i) $-\mathrm{x}-1 / 2,-\mathrm{y}+3 / 2,-\mathrm{z}+1$. 